Aus der Klinik für Kardiologie und Pneumologie

(Prof. Dr. med. G. Hasenfuß)

der Medizinischen Fakultät der Universität Göttingen

\title{
Batterielaufzeiten implantierbarer Kardioverter-Defibrillatoren
}

Eine retrospektive Analyse an der Universitätsmedizin Göttingen

\author{
INAUGURAL-DISSERTATION \\ zur Erlangung des Doktorgrades \\ der Medizinischen Fakultät der \\ Georg-August-Universität zu Göttingen
}

vorgelegt von

Matthias Lueken

aus

Bremen

Göttingen 2020 
Dekan:

Prof. Dr. Wolfgang Brück

Referent:

Prof. Dr. Markus Zabel

Ko-Referent/in:

PD Dr. Ulrich Krause

Drittreferent/in:

Prof. Dr. Margarete Schön

Tag der mündlichen Prüfung: 01.02 .2022 
Hiermit erkläre ich, die Dissertation mit dem Titel "Batterielaufzeiten implantierbarer Kardioverter-Defibrillatoren - Eine retrospektive Analyse an der Universitätsmedizin Göttingen" eigenständig angefertigt und keine anderen als die von mir angegebenen Quellen und Hilfsmittel verwendet zu haben.

Göttingen, den

(Unterschrift) 
Die Daten, auf denen die vorliegende Arbeit basiert, wurden teilweise publiziert:

Seegers J, Exposito PM, Lüthje L, Fischer T, Lueken M, Wenk H, Sossalla ST, Hasenfuss G, Zabel M (2015): Longevity of implantable cardioverter-defibrillators in a single-center population. J Interv Card Electrophysiol 44, 179-186 


\section{Inhaltsverzeichnis}

Abbildungsverzeichnis ...............................................................................

Tabellenverzeichnis .............................................................................................IV

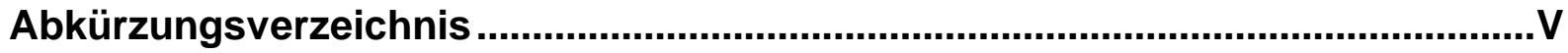

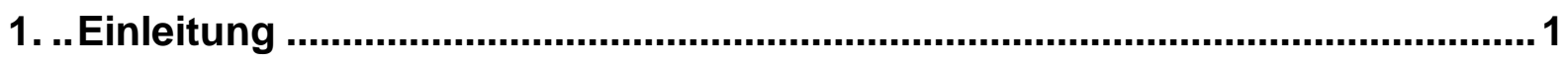

1.1 Geschichtliche Entwicklung der ICD-Indikation ...............................................

1.1.1 Studien zur Sekundärprophylaxe des plötzlichen Herztodes ........................2

1.1.2 Studien zur Primärprophylaxe des plötzlichen Herztodes .............................. 3

1.1.3 Kardiale Resynchronisationstherapie plus Defibrillator .................................. 4

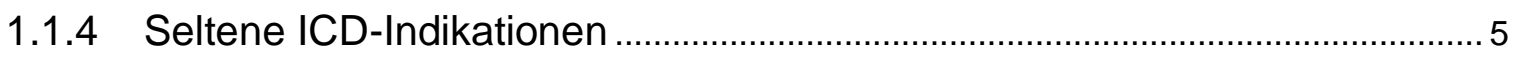

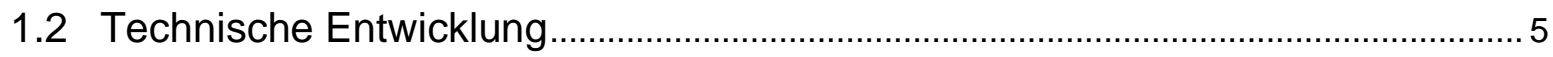

1.3 Stand der Forschung: Studien zu ICD-Batterielaufzeiten ..................................... 7

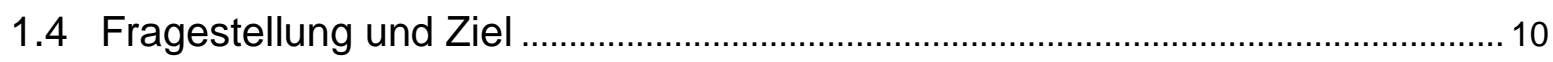

2. ...Material und Methoden ........................................................................ 11

2.1 Methodische Anlage der Untersuchung .......................................................... 11

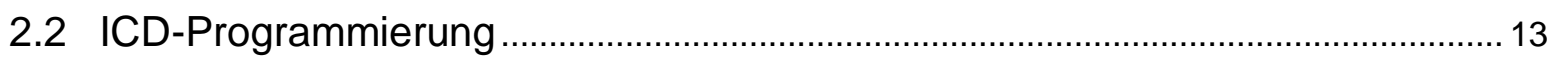

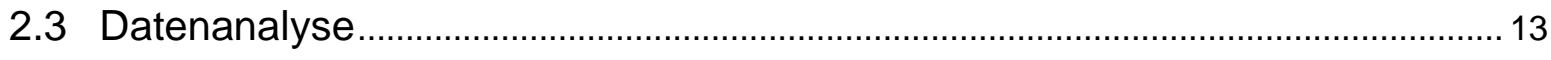

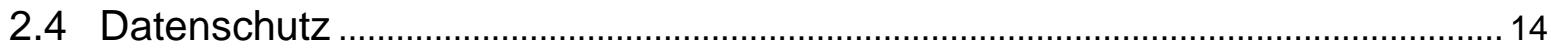

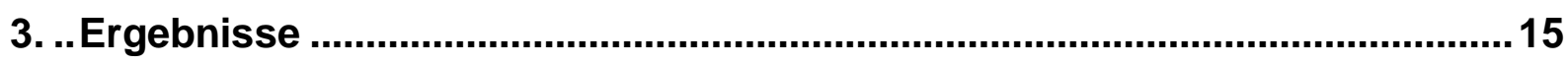

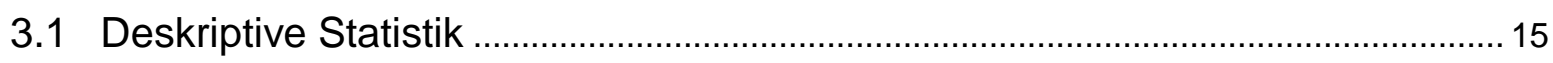

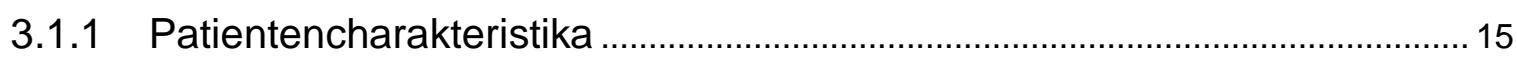

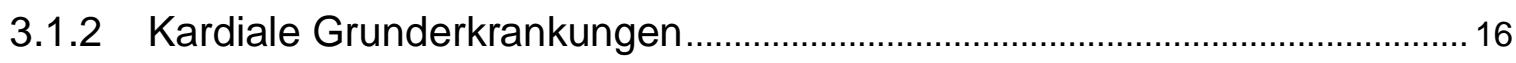

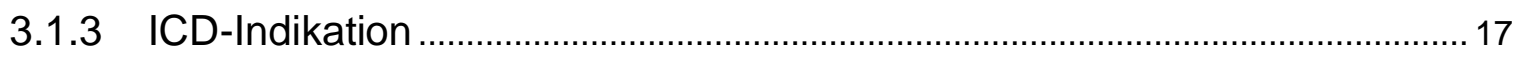

3.1.4 Kardiale Medikation bei Erstimplantation .................................................... 17

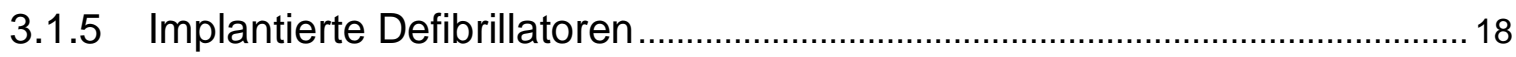


3.2 Batterielaufzeitanalysen 22

3.2.1 Einfluss des ICD-Herstellers auf die Batterielaufzeit....................................... 22

3.2.2 Einfluss des ICD-Typs auf die Batterielaufzeit ............................................... 27

3.2.3 Einfluss der Stimulation auf die Batterielaufzeit ............................................. 28

3.2.4 Einfluss der abgegebenen Schocks auf die Batterielaufzeit ......................... 30

3.2.5 Einfluss der ICD-Generation auf die Batterielaufzeit........................................ 31

3.2.6 Univariate Cox-Regressionsanalyse zur Batterielaufzeit ...............................35

3.2.7 Multivariate Cox-Regressionsanalyse zur Batterielaufzeit .............................36

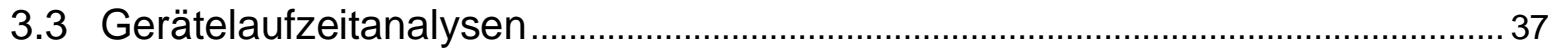

3.3.1 Einfluss des ICD-Herstellers auf die Gerätelaufzeit..........................................37

3.3.2 Einfluss des Kammertyps auf die Gerätelaufzeit.......................................... 38

4. ...Diskussion...................................................................................................... 41

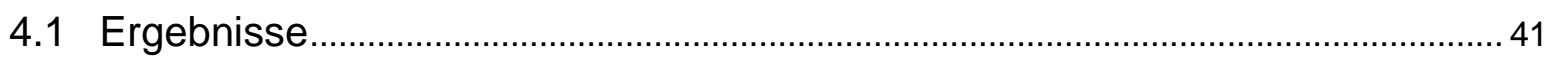

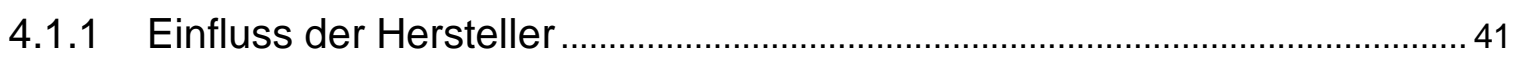

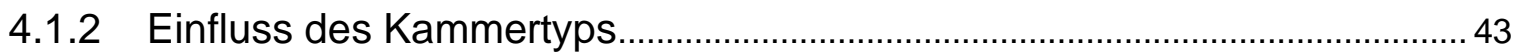

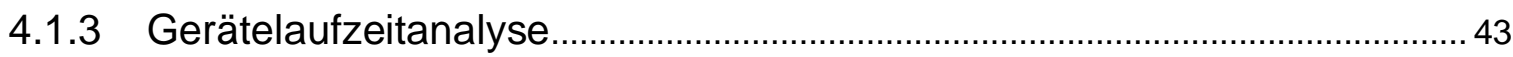

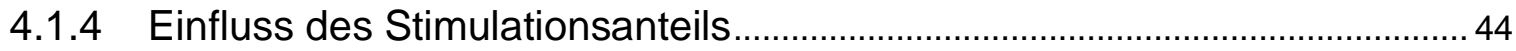

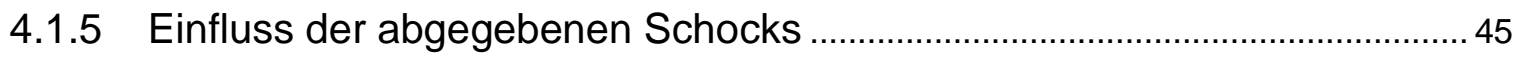

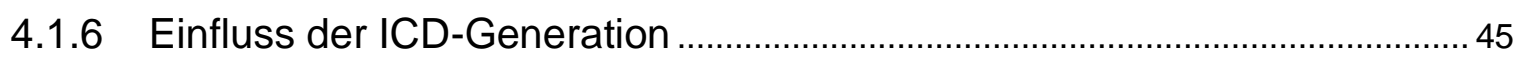

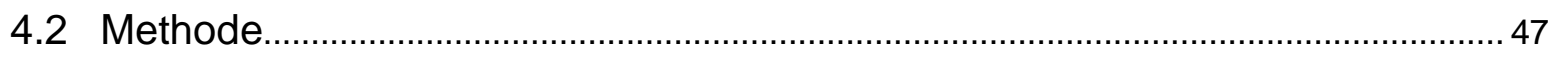

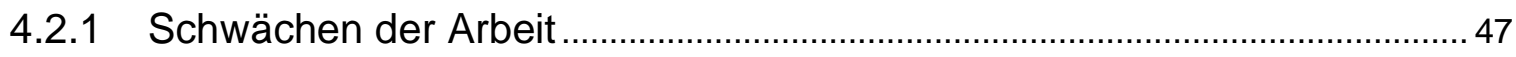

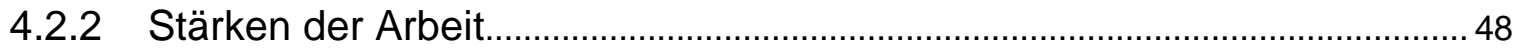

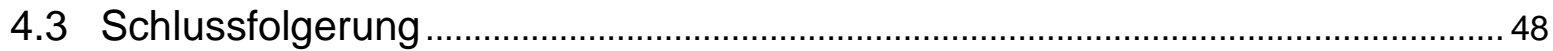

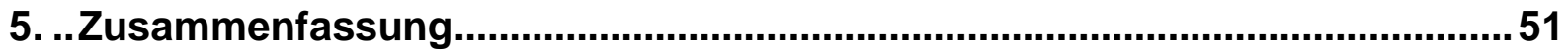

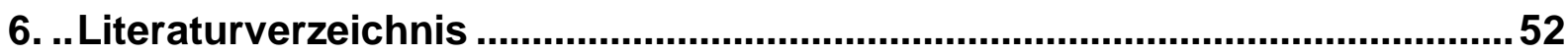




\section{Abbildungsverzeichnis}

Abbildung 1: Altersverteilung bei Erstimplantation 15

Abbildung 2: Einfluss des Herstellers auf die Batterielaufzeit ........................................................2 23

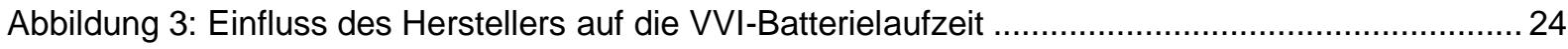

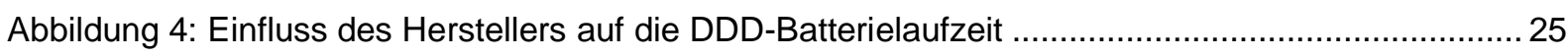

Abbildung 5: Einfluss des Herstellers auf die CRT-D-Batterielaufzeit ................................................. 26

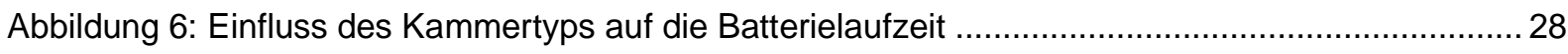

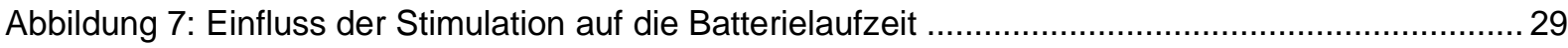

Abbildung 8: Einfluss der abgegebenen Schocks auf die Batterielaufzeit ........................................ 30

Abbildung 9: Einfluss der ICD-Generation auf die Batterielaufzeit .................................................... 33

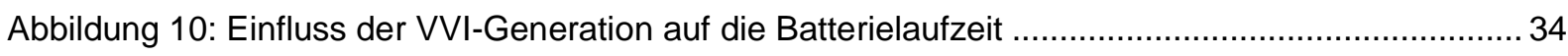

Abbildung 11: Einfluss des Herstellers auf die Gerätelaufzeit ........................................................... 38

Abbildung 12: Einfluss des Kammertyps auf die Gerätelaufzeit...................................................... 39 


\section{Tabellenverzeichnis}

Tabelle 1: Patientencharakteristika und kardiale Grunderkrankungen .............................................. 16

Tabelle 2: ICD-Indikation und kardiale Ereignisse vor Erstimplantation........................................... 17

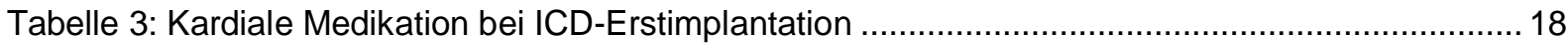

Tabelle 4: Gründe für Wechseloperationen ......................................................................... 19

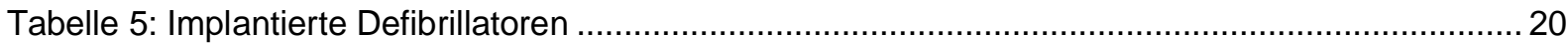

Tabelle 6: Eigenschaften der ICDs zum Einfluss des Herstellers auf die Batterielaufzeit ....................22

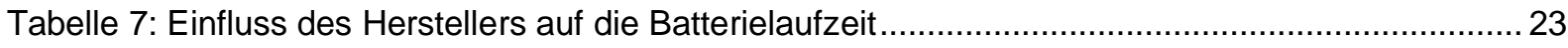

Tabelle 8: Einfluss des Herstellers auf die VVI-Batterielaufzeit......................................................... 24

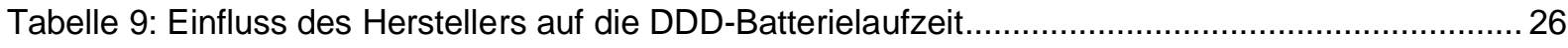

Tabelle 10: Einfluss des Herstellers auf die CRT-D-Batterielaufzeit ................................................ 27

Tabelle 11: Eigenschaften der ICDs zum Einfluss des Kammertyps auf die Batterielaufzeit ..............27

Tabelle 12: Einfluss des Kammertyps auf die Batterielaufzeit...................................................... 28

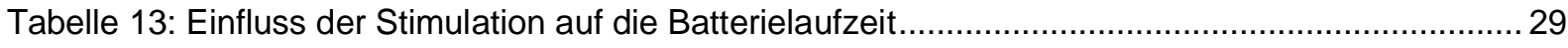

Tabelle 14: Einfluss der abgegebenen Schocks auf die Batterielaufzeit.......................................... 31

Tabelle 15: Eigenschaften der ICDs zum Einfluss der ICD-Generation auf die Batterielaufzeit ........... 32

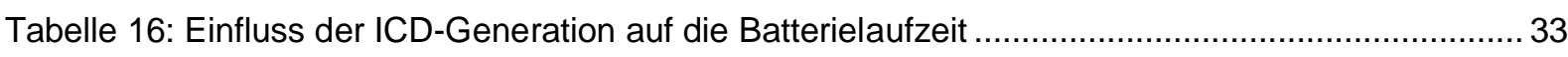

Tabelle 17: Univariate Cox-Regressionsanalyse zur Batterielaufzeit.................................................. 35

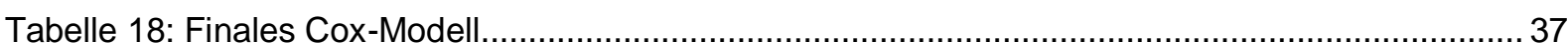

Tabelle 19: Einfluss des Herstellers auf die Gerätelaufzeit ............................................................. 38

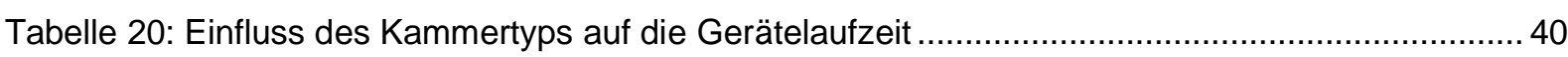




\section{Abkürzungsverzeichnis}

ARVC

ATP

AVID

$\mathrm{BIO}$

BSI

CRT-D

DCM

DDD-ICD

ERI

$\mathrm{HCM}$

$\mathrm{HR}$

ICD

KHK

$\mathrm{KI}$

LVEF

MED

NYHA

SJM

VF

VT

VVI-ICD
Arrhythmogene rechtsventrikuläre Kardiomyopathie

Antitachykarde Stimulation (Antitachykardia pacing)

Antiarrhythmics versus Implantable Defibrillators (Studie)

Biotronik

Boston Scientific / Guidant

Kardiale Resynchronisationstherapie-Defibrillator

Dilatative Kardiomyopathie

Dual-Dual-Dual-ICD (Zweikammer-Defibrillator)

Batterieerschöpfung (Elective replacement indicator)

Hypertrophe Kardiomyopathie

Hazard ratio

Implantierbarer Kardioverter-Defibrillator

Koronare Herzkrankheit

Konfidenzintervall

Linksventrikuläre Ejektionsfraktion

Medtronic

New York Heart Association

St. Jude Medical

Kammerflimmern

Ventrikuläre Tachykardie

Ventrikel-Ventrikel-inhibiert-ICD (Einkammer-Defibrillator) 


\section{Einleitung}

Es konnte in zwei Landmark-Studien überzeugend nachgewiesen werden, dass implantierbare Kardioverter-Defibrillatoren (ICDs) die Überlebenswahrscheinlichkeit bei bestimmten Patientengruppen verbessern (Moss et al. 2002; Bardy et al. 2005). Hieraus haben sich in den letzten 15-20 Jahren die Leitlinien der Primär- und Sekundärprophylaxe des plötzlichen Herztodes etabliert (Ponikowski et al. 2016; Priori et al. 2015), und die ICD-Implantation verbreitete sich rasch. In Deutschland wurden so beispielsweise im Jahr 2016 ca. 27000 Neuimplantationen registriert (Raatikainen et al. 2017, Hindricks et al. 2017). Obwohl als dauerhafte Therapie konzipiert, übersteigt die Lebenszeit der Patienten sehr häufig die Laufzeit der Geräte (Hauser 2005, Neuzner 2015). Eine Batterieerschöpfung des ICDs ist die Hauptursache für einen Aggregatwechsel, wobei jede Wechseloperation für die Patienten ein gewisses Operationsrisiko mit sich bringt. So liegt die Rate an komplikationsbedingten ReOperationen nach ICD-Implantationen bei Aggregatwechseln in etwa doppelt so hoch wie bei ICD-Erstimplantationen. Das Risiko für operationsbedingte Komplikationen, wie beispielsweise Tascheninfektionen oder Hämatome, steigt auch mit jedem weiteren Aggregatwechsel (Borleffs et al. 2010; Uslan et al. 2012; Johansen et al. 2011; Krahn et al. 2011; Poole et al. 2010; Gould und Krahn 2006). Die zunehmenden Implantationen und nachfolgenden Wechseloperationen stellen einen erheblichen Kostenpunkt für das Gesundheitssystem dar. So errechneten Cowie et al. (2009) beispielsweise die Kosten pro ICD-bedingt gewonnenem, qualitätskorrigiertem Lebensjahr (QALY, Quality-adjusted life year) auf $31717 €$. Würde laut einer Analyse von Gandjour et al. (2011) allen in Deutschland lebenden Patienten ein ICD implantiert, die nach der Studienlage (MADIT II-Studie, Moss et al. 2002) von einer ICDImplantation profitieren würden, würde dies mit einem Kostenanstieg zwischen 173 Millionen und 1,7 Milliarden € pro Jahr für das Gesundheitssystem einhergehen.

Das Ziel dieser Arbeit ist es, mögliche Einflussfaktoren, die mit einer verminderten ICDBatterielaufzeit einhergehen, zu identifizieren. Es sollte herausgearbeitet werden, ob sich in der Literatur beschriebene Unterschiede der Batterielaufzeiten bezüglich des ICD-Herstellers, des ICD-Typs, der Anzahl an abgegebenen Schocks und des prozentualen Stimulationsanteils anhand Göttinger Daten bestätigen lassen. 


\subsection{Geschichtliche Entwicklung der ICD-Indikation}

Mirowski et al. implantierten 1980 im Johns Hopkins Medical Center in Baltimore den ersten ICD einer Patientin, die zweimalig einen Herzstillstand als Äquivalent eines plötzlichen Herztodes überlebt hatte (Mirowski et al.1980). Die „Food and Drug Administration" der Vereinigten Staaten (FDA) gab 1985 ihre erste Empfehlung zur ICD-Implantation bei Patienten die mindestens einen plötzlichen Herzstillstand auf Grund einer hämodynamisch relevanten ventrikulären Tachyarrhythmie überlebt hatten (FDA 1985). Diesem historischen Ereignis folgten viele Studien zur Indikation einer ICD-Implantation, in denen die ICD-Therapie gegenüber anderen Therapieoptionen signifikant überlegen war. Diese werden im Folgenden dargestellt.

\subsubsection{Studien zur Sekundärprophylaxe des plötzlichen Herztodes}

Die erste randomisierte Studie zur Sekundärprophylaxe des plötzlichen Herztodes wurde von Wever et al. (1995) publiziert. Die Studie begann 1989 und randomisierte 60 Patienten in zwei Gruppen, die einen plötzlichen Herzstillstand auf Grund eines Myokardinfarktes überlebt hatten. Die Patienten der ersten Gruppe wurden einer ICDImplantation unterzogen $(n=29)$, die Patienten der zweiten Gruppe $(n=31)$ erhielten eine konventionelle Therapie, bestehend aus antiarrhythmischer Medikation und antiarrhythmischer Chirurgie. Die Gesamtmortalität in der ICD-Gruppe lag bei vier Patienten gegenüber elf Patienten in der Kontrollgruppe. Hiermit wurde erstmals in einer randomisierten Studie ein Überlebensvorteil durch eine ICD-Therapie gezeigt. Eine weitere Studie zur Sekundärprophylaxe des plötzlichen Herztodes war die AVIDStudie (Antiarrhythmics versus Implantable Defibrillators Trial). In dieser Multicenterstudie konnte bei 1016 Patienten, die entweder einen plötzlichen Herztod oder anhaltende symptomatische Tachykardien überlebt hatten, ein signifikanter Überlebensvorteil durch eine ICD-Therapie gegenüber einer antiarrhythmischen Medikation gezeigt werden (relative Mortalitätsreduktion um 29 \%) (AVID Investigators 1997). Die CIDS-Studie (Canadian Implantable Defibrillator Study) untersuchte 659 Patienten die entweder ein Kammerflimmern oder eine anhaltende ventrikuläre Tachykardie überlebt hatten. Hierbei konnte eine relative Risikoreduktion der arrhythmogen bedingten Mortalität um $33 \%$ durch eine ICD-Therapie im Vergleich mit einer Amiodarontherapie registriert werden (Connolly et al. 2000a; Sheldon et al. 2000). 
Auch die CASH-Studie (Cardiac Arrest Study Hamburg) untersuchte Patienten ( $\mathrm{n}=$ 288), die einen plötzlichen Herztod mit dokumentiertem Kammerflimmern überlebt hatten. Eine ICD-Implantation ging bei diesem Patientenkollektiv mit einer Reduktion der Gesamtmortalität um 23 \% gegenüber einer antiarrhythmischen Therapie mit Metoprolol / Amiodaron einher. Dieses Ergebnis war aber statistisch nicht signifikant (Kuck et al. 2000).

Die beschriebenen Studien zur Sekundärprophylaxe des plötzlichen Herztodes boten auch eine Grundlage für Metaanalysen. In diesen wurde bestätigt, dass eine ICDTherapie nach einem plötzlichen Herztod im Vergleich mit einer medikamentösen Therapie einen signifikanten Überlebensvorteil darstellt. Die ICD-Therapie senkt sowohl das Risiko für einen arrhythmogen bedingten Tod, als auch die Gesamtmortalität (Connolly et al. 2000b; Lee et al. 2003; Ezekowitz et al. 2003).

\subsubsection{Studien zur Primärprophylaxe des plötzlichen Herztodes}

Die Patienten der genannten Studien hatten eine maligne Arrhythmie überlebt und somit ein hohes Risiko für ein erneutes malignes kardiales Ereignis (Priori et al. 1995; 1997). Patienten mit ähnlichen Grunderkrankungen haben aber auch ohne stattgehabten plötzlichen Herztod ein hohes Risiko, einen plötzlichen Herztod zu erleben. Deshalb haben sich einige Studien mit der primärprophylaktischen Implantation eines ICDs in Hochrisikopatienten befasst. Das Ziel hierbei war es, diese Hochrisikopatienten näher zu definieren und eine evidenzbasierte ICD-Indikation festzulegen.

Die MADIT-Studie (Multicenter Automatic Defibrillator Implantation Trial) war die erste Studie zur Primärprophylaxe des plötzlichen Herztodes. Es konnte ein signifikanter Überlebensvorteil durch eine ICD-Therapie im Vergleich mit einer medikamentösen Therapie bei 196 Hochrisikopatienten gezeigt werden. Einschlusskriterien waren ein erlebter Myokardinfarkt sowie eine nicht anhaltende ventrikuläre Tachykardie, eine reduzierte linksventrikuläre Ejektionsfraktion (LVEF) $<35 \%$ und induzierbare, nicht unterdrückbare ventrikuläre Tachyarrhythmien in der elektrophysiologischen Untersuchung (Moss et al. 1996).

In der MUSTT-Studie (Multicenter Unsustained Tachycardia Trial) wurden 704 Patienten mit induzierbaren ventrikulären Tachykardien, einer koronaren Herzkrankheit (KHK) sowie einer LVEF von unter $40 \%$ in Gruppen randomisiert. Gegenüber keiner Therapie beziehungsweise einer elektrophysiologisch kontrollierten 
antiarrhythmischen Therapie konnte durch eine ICD-Implantation bei diesen Patienten eine signifikante Reduktion der Mortalität festgestellt werden (Buxton et al. 1999).

Auch die MADIT II-Studie (Multicenter Automatic Defibrillator Implantation Trial II) befasste sich mit der primärprophylaktischen ICD-Implantation, wobei hier erstmals auf jegliche elektrophysiologische Testung zur Risikostratifizierung verzichtet wurde. Bei Patienten, die einen Myokardinfarkt überlebt und eine stark eingeschränkte LVEF von unter $30 \%$ hatten, konnte durch eine primärprophylaktische ICD-Implantation eine Reduktion der Gesamtmortalität um 31 \% im Vergleich mit konventionell behandelten Patienten gezeigt werden (Moss et al. 2002).

Anders als diese Studien untersuchte die SCD-HeFT-Studie (Sudden Cardiac Death in Heart Failure Trial) nicht nur Patienten mit ischämischer Herzerkrankung, sondern auch nichtischämische Herzerkrankungen, wie beispielsweise die dilatative Kardiomyopathie (DCM). Ähnlich der MADIT II-Studie wurde auf sämtliche Arrhythmiekriterien verzichtet und nur Patienten mit einer symptomatischen Herzinsuffizienz (NYHA II und III) und einer LVEF von unter $35 \%$ eingeschlossen. Durch eine primärprophylaktische ICD-Implantation konnte im Vergleich mit einer Amiodaron- / Placebo-Therapie das relative Mortalitätsrisiko um 23 \% gesenkt werden (Bardy et al. 2005).

\subsubsection{Kardiale Resynchronisationstherapie plus Defibrillator}

Auch die primärprophylaktische Implantation von Defibrillatoren kombiniert mit kardialer Resynchronisationstherapie (CRT-D) konnte in Studien Vorteile zeigen. In der COMPANION-Studie (Comparison of Medical Therapy, Pacing, and Defibrillation in Heart Failure) war eine CRT-D-Implantation bei Patienten mit einer fortgeschrittenen Herzinsuffizienz (NYHA III und IV) und einer verlängerten QRS-Zeit von mindestens 120 ms mit einer Reduktion der Mortalität um 36 \% im Vergleich mit einer rein medikamentösen Therapie verbunden (Bristow et al. 2004). Bei einer ähnlichen Patientenpopulation (Herzinsuffizienz NYHA III und IV, LVEF < $35 \%$ und QRS > $130 \mathrm{~ms}$ ) war in der MIRACLE-Studie (Multicenter InSync Randomized Clinical Evaluation) eine CRT-D-Therapie mit einer Verbesserung der Lebensqualität im Vergleich mit einer Kontrollgruppe verbunden. So verbesserte sich die LVEF und die Strecke, die die Patienten in sechs Minuten gehen konnten (Abraham et al. 2002; Young et al. 2003). Im Vergleich mit einer ICD-Implantation führte eine CRT-DTherapie in der MADIT-CRT-Studie (Multicenter Automatic Defibrillator Implantation 
Trial with Cardiac Resynchronization Therapy) zu einer Reduktion kardialer Dekompensationen und Verbesserung der linksventrikulären Ejektionsfraktion. Ein statistisch signifikanter Überlebensvorteil durch die CRT-D-Therapie gegenüber der ICD-Therapie konnte jedoch nicht nachgewiesen werden (Moss et al. 2009).

\subsubsection{Seltene ICD-Indikationen}

Die Patienten der bisher vorgestellten Untersuchungen litten an einer KHK oder einer DCM als kardialer Grunderkrankung und hatten dadurch ein erhöhtes Risiko für einen plötzlichen Herztod. Neben diesen in der Bevölkerung häufigen Grunderkrankungen gibt es verhältnismäßig seltene Erkrankungen, die ebenfalls ein erhöhtes Risiko für einen plötzlichen Herztod aufweisen (Priori et al. 2001). Hierzu zählt beispielsweise die hypertrophe Kardiomyopathie (HCM), für die in der Literatur ein Überlebensvorteil durch eine ICD-Therapie gezeigt wurde (Begley et al. 2003; Maron et al. 2000). Gleiches gilt u. a. für das Long-QT-Syndrom (Kaufman 2003; Zareba et al. 2003), das Brugada-Syndrom (Littmann et al. 2003; Priori et al. 2002), die arrhythmogene rechtsventrikuläre Kardiomyopathie (ARVC) (Corrado et al. 2003; 2010; Tavernier et al. 2001), bestimmte kongenitale Vitien (Shannon 2002) und höhergradige Herzklappenerkrankungen (Priori et al. 2001).

\section{$1.2 \quad$ Technische Entwicklung}

Parallel zur Weiterentwicklung der ICD-Indikation unterlagen auch die technischen Aspekte der ICD-Therapie einem stetigen Fortschritt. Vom ersten implantierten ICD bis zu den ICDs der aktuellen Generation wurden die Geräte kontinuierlich weiterentwickelt, z. B. von einem Einkammer-Defibrillator zu immer komplexeren Geräten mit einer Vielzahl therapeutischer und diagnostischer Möglichkeiten (Cannom und Prystowsky 2004). Die ersten ICDs wurden abdominal implantiert und mit epikardialen Patch-Elektroden über dem Herzen verbunden um Arrhythmien zu erkennen und gegebenenfalls Defibrillationsschocks abzugeben. Zur Anlage war eine Thorakotomie erforderlich und die Aggregate waren vergleichsweise groß. Seit dem Jahr 1988 konnten transvenöse Systeme implantiert werden und auf eine Thorakotomie verzichtet werden, was eine erhebliche Absenkung des operativen Risikos zur Folge hatte (Saksena und Parsonnet 1988; Zipes und Roberts 1995).

Als weitere Neuerung wurde die Möglichkeit der antibradykarden Stimulation (Pacing) eingeführt. Hierdurch konnte die bis dahin häufig bei ICD-Patienten zusätzlich 
notwendige Schrittmacherimplantation vermieden werden. Die Einführung der biphasischen Schockabgabe sowie die Entwicklung des sogenannten „Hot-CanPrinzips" führte zu deutlich niedrigeren Defibrillationsschwellen (Bardy et al. 1989; 1993). Beim „Hot-Can-Prinzip“ wird bei einem Schock ein elektrisches Feld zwischen dem Wendel (coil) am distalen Ende der ICD-Sonde und dem eigentlichen ICDGehäuse gebildet. Die Geräte konnten nunmehr auch R-R-Intervalle von Arrhythmieepisoden aufzeichnen, wodurch sich retrospektiv Diagnosen stellen liessen. Diese Speicherkapazitäten werden seither stetig ausgebaut (Sarter et al. 1998; Swygman et al. 2002). Es folgten weitere Entwicklungen, wie z. B. die Möglichkeit, schnelle ventrikuläre Tachykardien mittels antitachykarder Stimulation (ATP) oder der Abgabe von Schocks niedriger Energie zu beenden, bevor diese in ein Kammerflimmern übergehen (Cannom und Prystowsky 2004).

Durch immer kleinere Geräte konnte sich die pektorale gegenüber der abdominalen Implantation als Standard bei der ICD-Implantation durchsetzen (Hoffmann und Steinbeck 1998). Neben dem Einkammer-Defibrillator (VVI), dessen Sonde im rechten Ventrikel verankert ist, wurden weitere ICD-Typen eingeführt. Der ZweikammerDefibrillator (DDD), der jeweils eine Sonde im rechten Ventrikel und im rechten Vorhof hat, und somit zur besseren Differenzierung von supraventrikulären und ventrikulären Tachykardien beiträgt, konnte erste Erfolge zeigen (Lavergne et al. 1997). Außerdem wurden biventrikuläre Schrittmacher zur kardialen Resynchronisationstherapie mit einem Defibrillator kombiniert (CRT-D) (Higgins et al. 2000). Diese sind mit einer Sonde im rechten Ventrikel und einer Sonde im rechten Vorhof ausgestattet. Eine dritte Sonde liegt dem linken Ventrikel von außen über den Koronarsinus an. Darüber hinaus gibt es seit kurzer Zeit subkutane ICDs, mit denen erste positive Erfahrungen gemacht wurden (Dabiri Abkenari et al. 2011).

Bezüglich der Batterien waren die ersten ICD-Geräte mit Lithium-VanadiumpentoxidBatterien ausgestattet, welche ein insgesamt unzuverlässiges Entladeverhalten hatten und zu spontanen Selbstentladungen neigten. Später wurden verschiedene Batterietypen wie die Lithium-Silber-Vanadiumoxid-Batterie, die LithiumMangandioxid-Batterie, oder die Lithium-Silber-Vanadiumoxid / Kohlenstoffmonofluorid-Hybrid-Batterie eingeführt, welche sich in ihren Eigenschaften etwas unterscheiden und je nach Hersteller und Typ in den ICDs der heutigen Generation zu finden sind (Mond und Freitag 2014).

Zusammenfassend werden in immer kürzerer Zeit neue Geräte hergestellt und es werden stetig neue Algorithmen und Diskriminationskriterien zur technischen 
Verbesserung der ICDs entwickelt (Van Welsenes et al. 2011a). Der Trend zu kleineren Geräten schien zunächst nicht mit einer verbesserten Batterielaufzeit verbunden zu sein (Johansen et al. 2016).

\subsection{Stand der Forschung: Studien zu ICD-Batterielaufzeiten}

Aussagen zu Batterielaufzeiten von implantierbaren Kardioverter-Defibrillatoren stützten sich überwiegend auf die Angaben der Hersteller. In den letzten Jahren fanden jedoch erstmals unabhängige, klinische Untersuchungen zu diesem Thema statt. Diese Arbeiten werden im Folgenden vorgestellt.

Biffi et al. (2008) untersuchten 153 Patienten, die im Zeitraum von Januar 2000 bis Dezember 2002 an der Universitätsklinik Bologna einen ICD erhielten. Es konnte gezeigt werden, dass VVI-ICDs und ICDs der Firma Medtronic, im Gegensatz zu ICDs der Firmen Guidant und St. Jude Medical, die niedrigste batteriebedingte Wechselrate hatten.

Schaer et al. (2009) analysierten alle von 1994 bis Juli 2007 an der Universitätsklinik Basel implantierten ICDs $(\mathrm{n}=644)$ hinsichtlich Einflussfaktoren auf ICDBatterielaufzeiten. Es zeigte sich, dass es erhebliche Differenzen zwischen den von den Herstellern angegebenen und den tatsächlichen Batterielaufzeiten gab. So waren nach 5 Jahren nur noch $70 \%$ der implantierten ICDs aktiv. Laut Herstellerangabe hätten es $80 \%$ sein müssen. Außerdem konnten deutliche Unterschiede in der Batterielaufzeit zwischen den Herstellern gefunden werden: Medtronic-ICDs liefen im Median 7,6 Jahre, Boston-Scientific- / Guidant-ICDs 5 Jahre und St.-Jude-ICDs 3,8 Jahre.

Um die tatsächliche Lebensdauer der ICDs zu messen untersuchten Horlbeck et al. (2012) retrospektiv alle zwischen Juni 1988 und Juni 2009 implantierten ICDs ( $n=$ 1502) der Universitätsklinik Bonn. Es wurden alle ICD-Aggregatwechsel berücksichtigt, egal ob diese batteriebedingt oder aus anderen Gründen durchgeführt wurden. Geräte der Firma Medtronic zeigten hier ebenfalls im Vergleich mit anderen Herstellern die längsten Laufzeiten bis zum Aggregatwechsel (im Mittel 5,5 Jahre). Ferner zeigten CRT-D-Geräte die kürzeste und VVI-Geräte die längste Laufzeit im Vergleich der verschiedenen Kammertypen.

In einer großen monozentrischen Arbeit von Thijssen et al. (2012) wurde die Lebensdauer von 4673 ICDs, die zwischen 1996 und April 2011 an der Universitätsklinik Leiden implantiert wurden, untersucht. Die mittlere Batterielaufzeit 
aller Geräte betrug 5,5 \pm 0,1 Jahre. Im Vergleich der Kammertypen zeigten DDDGeräte die längste Batterielaufzeit (MW: 5,8 \pm 0,1 Jahre) vor VVI- (MW: 5,5 \pm 0,2 Jahre) und CRT-D-Geräten (MW: 4,7 \pm 0,1 Jahre). Neuere Geräte, die nach 2002 implantiert wurden, wiesen eine längere Batterielaufzeit auf als Geräte, die vor 2002 implantiert wurden (MW: 5,6 \pm 0,1 Jahre vs. MW: 4,9 0,2 Jahre). Geräte der Firma Medtronic zeigten die längste Batterielaufzeit (MW: 5,8 Jahre), gefolgt von Geräten der Firma Boston Scientific (MW: 5,3 \pm 0,1 Jahre); St. Jude Medical (MW: $5 \pm$ 0,2 Jahre) und Biotronik (MW: 4,7 \pm 0,1 Jahre). Die Anzahl abgegebener Schocks hatte keinen nachweisbaren Einfluss auf die Batterielaufzeit.

Alam et al. (2014) verglichen die Batterielaufzeiten von 646 CRT-Ds verschiedener Hersteller miteinander, welche von Januar 2008 bis Dezember 2010 an der Universitätsklinik Pittsburgh implantiert wurden. Während der Nachsorge von 2,7 $\pm 1,5$ Jahren mussten $25 \%$ der Medtronic-Geräte gegenüber $4 \%$ der Boston-Scientific- und $7 \%$ der St.-Jude-Medical-CRT-Ds batteriebedingt ausgetauscht werden. Somit war die 4-Jahres-Überlebensrate der ICD-Batterien von Medtronic-Geräten mit $67 \%$ signifikant kürzer $(\mathrm{p}<0,001)$, als die der anderen beiden untersuchten Hersteller (BSI 94 \%; SJM $92 \%)$.

Zu einem ähnlichen Ergebnis kamen Landolina et al. (2015). Sie untersuchten in einer Multicenterstudie die Batterielaufzeiten von 1726 CRT-Ds die an neun italienischen Zentren im Zeitraum von Januar 2008 bis März 2010 implantiert wurden. Die 5-JahresBatterielaufzeit von Medtronic-CRT-Ds lag hier mit $42 \%$ deutlich unter der von BSICRT-Ds (66 \%). Im Generationenvergleich (Markteinführung vor 2007 vs. ab 2007) waren diese Unterschiede noch deutlicher zu sehen (Implantation ab 2007: Medtronic $52 \%$ vs. BSI $88 \%$ ). Darüber hinaus waren eine hohe linksventrikuläre Stimulationsausgabe ebenso wie eine unipolare Stimulationskonfiguration mit einem erhöhten Risiko für batteriebedingte Aggregatwechsel assoziiert.

Von Gunten et al. (2016) analysierten die Batterielaufzeiten von 4881 ICDs die zwischen März 1994 und Januar 2014 an der Universitätsklinik in Rotterdam und der Universitätsklinik in Basel implantiert wurden. Im Generationenvergleich zeigten ICDs, die vor 2006 implantiert wurden, herstellerabhängig kürzere Batterielaufzeiten als ICDs, die ab dem Jahr 2006 implantiert wurden. Ältere VVI- und DDD-Geräte des Herstellers Medtronic sowie ältere CRT-Ds des Herstellers St. Jude Medical zeigten die längsten Batterielaufzeiten. Bei den neueren ICDs zeigten Geräte aller Kammertypen des Herstellers Boston Scientific / Guidant die längsten Batterielaufzeiten. Darüber hinaus lag, nur die VVI-Geräte betrachtet, das 5-Jahres- 
Überleben der Patienten $5 \%$ über den 5-Jahres-Batterielaufzeiten. Bei DDD-Geräten betrug dieser Unterschied schon $15 \%$ und bei CRT-Ds lag er bei $23 \%$.

Zanon et al. (2016) konnten in ihrer Batterielaufzeitanalyse von 953 ICDs, die an insgesamt 36 italienischen Zentren im Zeitraum von 2002 bis 2014 implantiert wurden, ebenfalls herstellerbedingte Batterielaufzeitunterschiede zeigen. VVI- und DDDGeräte des Herstellers St. Jude Medical hatten die längsten medianen Batterielaufzeiten (6,7 Jahre) gegenüber VVI- und DDD-ICDs des Herstellers Biotronik (5,3 Jahre). Dazwischen lagen VVI- und DDD-Geräte der folgenden Hersteller mit absteigender medianer Batterielaufzeit: Medtronic 6,4 Jahre, Sorin 6,4 Jahre, BSI 6,3 Jahre. In der separaten Betrachtung der CRT-Ds zeigten Geräte des Herstellers BSI mit im Median 5,8 Jahren die längsten Batterielaufzeiten, gefolgt von St. Jude Medical (5 Jahre), Sorin (5 Jahre), Medtronic (4,5 Jahre) und Biotronik (4,4 Jahre). Darüber hinaus waren ein hoher Stimulationsanteil sowie CRT-Ds mit einer hohen linksventrikulären Stimulationsausgabe mit einem signifikant erhöhten Risiko für batteriebedingte Aggregatwechsel verbunden. Auch in dieser Analyse wurde eine Gerätelaufzeituntersuchung durchgeführt, bei der sich insgesamt deutlich kürzere mediane Gerätelaufzeiten als Batterielaufzeiten zeigten (VVI und DDD: 5,9 Jahre; CRT-D: 4,9 Jahre).

Boriani et al. (2016) untersuchten die mittels Home Monitoring in der europäischen Datenbank „LATITUDE ${ }^{\circledR}$ " gesammelten Daten von 4851 ICDs des Herstellers BSI auf Einflussfaktoren zu ICD-Batterielaufzeiten. Es konnte ein Einfluss der Anzahl abgegebener Schocks auf die von den Geräten angegebene voraussichtliche Batterielaufzeit gezeigt werden. Darüber hinaus scheint eine hohe Stimulationsausgabe und ein hoher Stimulationsanteil bei CRT-D-Geräten mit vermehrtem Batterieverbrauch einherzugehen. Insgesamt gaben die Geräte der aktuellen Generation lange voraussichtliche Laufzeiten an, welche sich je nach Kammertyp unterschieden (VVI: 13 Jahre, DDD: 12 Jahre CRT-D: >10 Jahre). Es handelt sich hierbei allerdings um geschätzte, voraussichtliche Werte und keine realen, retrospektiv analysierten Daten.

Manolis et al. (2017) konnten in einer Analyse von 685 Patienten aus mehreren griechischen Zentren, welche in den letzten 20 Jahren dort einen ICD implantiert bekommen hatten, ebenfalls herstellerspezifische Unterschiede in den ICDBatterielaufzeiten dokumentieren. So hatten ICDs des Herstellers Medtronic die längsten Batterielaufzeiten (70,5 $\pm 21,6$ Monate) gegenüber ICDs der Hersteller BSI (58,3 $\pm 16,3$ Monate), ELA Medical (57,4 $\pm 12,5$ Monate), Biotronik (51,9 $\pm 13,5$ 
Monate) und St. Jude Medical (51,7 $\pm 15,4$ Monate). CRT-Ds zeigten im Vergleich mit VVI- und DDD-Geräten die kürzesten Batterielaufzeiten (CRT-D: $46.3 \pm 10$ Monate vs. DDD: $59 \pm$ 16,3 Monate vs. VVI: 63,6 $\pm 21,6$ Monate). In einem Vergleich der ICDGenerationen hatten ICDs der neueren Dekade längere Batterielaufzeiten als die ältere ICD-Generation (Neu: 60,4 $\pm 18,8$ Monate vs. Alt: 53,5 \pm 17,5 Monate).

\section{$1.4 \quad$ Fragestellung und Ziel}

Das Ziel dieser retrospektiven statistischen Arbeit war es, Einflussfaktoren auf die Batterielaufzeit von implantierbaren Kardioverter-Defibrillatoren zu identifizieren.

Die unter Kapitel 1.1.1 und 1.1.2 beschriebenen Studien konnten einen signifikanten Überlebensvorteil durch die ICD-Therapie in der Primär- und Sekundärprophylaxe des plötzlichen Herztodes zeigen. Folglich wurden in den letzten Jahren zunehmend mehr ICDs implantiert. Der häufigste Grund für ICD-Wechseloperationen ist eine Batterieerschöpfung. Wechseloperationen stellen sowohl einen mit Risiken für den Patienten behafteten operativen Eingriff dar und sind zum anderen ein erheblicher Kostenpunkt für das Gesundheitssystem. Um das Kosten-Nutzen-Verhältnis der ICDTherapie zu verbessern und die Anzahl operativer Eingriffe für die Patienten zu minimieren ist es notwendig die Batterielaufzeiten von ICDs zu verbessern. Dafür müssen Parameter eruiert werden, die die Batterielaufzeit signifikant beeinflussen.

In dieser Arbeit wurden u. a. folgende potentielle Einflussfaktoren auf die Batterielaufzeit von ICDs untersucht: Die ICD-Hersteller, der ICD-Kammertyp, die Anzahl an abgegebenen Schocks, der prozentuale Anteil an antibradykarder

Stimulation (Pacing percentage), die Generation der Geräte (Implantationsdatum vor/nach 2002) sowie kardiale Grunderkrankungen der Patienten und die Indikation zur ICD-Implantation. Außerdem wurde der potentielle Einfluss einiger der genannten Faktoren auf die reale Lebensdauer von ICDs untersucht. 


\section{Material und Methoden}

\subsection{Methodische Anlage der Untersuchung}

Es handelte sich bei der vorliegenden Untersuchung um eine retrospektive monozentrische statistische Analyse. Die Grundlage hierfür bot eine prospektive Datenbank der ICD-Ambulanz der Universitätsmedizin Göttingen. Alle Patienten, denen an der Universitätsmedizin Göttingen zwischen März 1987 und Oktober 2010 ein ICD oder ein CRT-D implantiert wurde, wurden hier untersucht und nachgesorgt. Die Nachsorgetermine und Aggregatkontrollen fanden regelmäßig im Abstand von drei bis sechs Monaten statt. Sie wurden über den gesamten Zeitraum im Wesentlichen von einer Person durchgeführt. Für jeden Patienten besteht die Datenbank aus folgenden Quellen: Patientenakte, digitales Krankenhausinformationssystem, ICDImplantationsprotokolle und Nachsorgeprotokolle.

Von jedem Patienten wurden medizinische Daten aufgenommen, beispielsweise die Indikation zur ICD-Implantation, kardiale Grunderkrankungen und die bestehende Medikation zum Zeitpunkt der Implantation. Die myokardiale Pumpfunktion gemessen durch die linksventrikuläre Ejektionsfraktion und das klinische Ausmaß der Herzinsuffizienz (NYHA-Klassifikation) wurden zusätzlich bestimmt. Bezüglich der Geräteparameter wurden der prozentuale Stimulationsanteil bei Erstimplantation und beim letzten Nachsorgetermin, sowie die Anzahl und Qualität der abgegebenen Schocks pro Patient erfasst. Für die Batterielaufzeitanalyse wurde die Gesamtzahl aller abgegebenen Schocks aller Geräte eines Patienten gemittelt und auf alle ICDs dieses Patienten verteilt. Je nach Anzahl abgegebener Schocks wurden die Geräte in folgende vier Gruppen eingeteilt: kein Schock, 1 Schock, 2-4 Schocks, 5 oder mehr Schocks.

Jeder Aggregatwechsel wurde dokumentiert und die Gründe hierfür in batteriebedingt und nicht batteriebedingt unterteilt. Batterieerschöpfung wurde dokumentiert, wenn die Batteriespannung des Aggregates den aggregatspezifischen Austauschwert erreicht oder unterschritten hatte. Dies wurde auch daran erkannt, dass der Batteriezustand während der Aggregatabfrage mit elective replacement indicator (ERI) oder recommended replacement time (RRT) bezeichnet wurde. Die ICD-Batterielaufzeit wurde als Zeit zwischen dem Implantationsdatum und dem Tag des Aggregatwechsels definiert. Gründe für nicht batteriebedingte Wechseloperationen waren beispielsweise ein System-Upgrade, Infektionen und Fehler der elektrischen Komponenten (Sonden, Konnektor, Sensor). Ein System-Upgrade wurde angegeben, wenn beispielsweise ein 
VVI-Gerät durch ein DDD-Gerät oder CRT-D-Gerät ersetzt wurde, oder wenn ein DDDGerät durch ein CRT-D-Gerät ersetzt wurde. Eine Batterieerschöpfung des ausgetauschten Gerätes konnte hierbei ebenfalls vorliegen. Die Laufzeitanalysen wurden sowohl für batteriebedingte Aggregatwechsel als auch für Wechseloperationen aus jedem Grund durchgeführt. Letztere können einen Hinweis auf die reale Lebensdauer des einzelnen ICDs geben.

Der mittlere antibradykarde Stimulationsanteil der letzten Nachsorge wurde für die statistische Auswertung verwendet. Die prozentualen Stimulationsanteile jeder Sonde wurden im Falle von DDD- oder CRT-D-Geräten addiert. Daraus resultiert bei VVIICDs ein Maximum von $100 \%$ Stimulation. Durch die Möglichkeit der Stimulation im rechten Ventrikel und im rechten Vorhof konnten bei DDD-Geräten maximal $200 \%$ Stimulation erreicht werden. Ein CRT-D kann durch Stimulation im rechten Ventrikel, rechten Vorhof und linken Ventrikel ein Maximum von $300 \%$ Stimulation erreichen. Für die Auswertung wurde der prozentuale Stimulationsanteil in drei Gruppen kategorisiert: $\leq 50 \%, 51-150 \%, \geq 151 \%$.

ICD-Geräte von folgenden vier großen ICD-Herstellen wurden im unregelmäßigen Wechsel implantiert: Geräte der Firma Biotronik (BIOTRONIK SE \& Co. KG - Berlin, Deutschland), der Firma Medtronic (Medtronic Inc. - Fridley, MN, USA), der Firma Guidant Corporation/ Boston Scientific (Guidant Corporation/ Boston Scientific Natick, MA, USA) sowie der Firma St. Jude Medical (St. Jude Medical - St. Paul, MN, USA). Von der Firma St. Jude Medical wurden insgesamt nur drei Geräte implantiert, sodass eine Auswertung hierfür nicht repräsentativ war. Geräte der Firma Biotronik wurden an der Universitätsmedizin Göttingen ab dem Jahr 2006 implantiert.

Um den Einfluss der ICD-Generation auf die Batterielaufzeit zu untersuchen, wurden die Geräte in zwei Gruppen kategorisiert: „Implantation vor 2002“ und „Implantation ab 2002". Dies geschah zum einen mit allen drei Gerätetypen und wurde darüber hinaus noch separat für VVI-ICDs untersucht. Hierdurch sollte ein potentieller Einfluss der vermehrt nach 2002 implantierten CRT-D-Geräte auf die Batterielaufzeit in der Gruppe der neueren ICDs vermindert werden. Des Weiteren konnte analysiert werden, ob sich die Batterielaufzeiten alter und neuer VVI-Geräte unterscheiden.

Einige Patienten wechselten nach einer ICD-Implantation das Nachsorgezentrum von der Universität Göttingen zu externen Häusern. Die Daten dieser Patienten wurden telefonisch nachverfolgt und ebenfalls in die Datenbank eingefügt. Ein entsprechender Ethikantrag (Antragsnummer 30/10/11) wurde diesbezüglich von der Ethikkommission 
genehmigt. Die Mortalitätsdaten der Patienten wurden durch eine Abfrage der jeweiligen Einwohnermeldeämter erhoben.

\subsection{ICD-Programmierung}

Die Programmierung der ICDs wurde individuell an die Bedürfnisse der Patienten angepasst, es lässt sich aber ein klinikinterner Standard beschreiben. Dieser wurde über ca. 15 Jahre von einer spezialisierten Krankenschwester durchgeführt und im Verlauf den jeweiligen aktuellen Empfehlungen und Leitlinien angepasst. Hierzu gehört, dass während jeder Nachsorgeuntersuchung Routine-Parameter abgefragt und grundlegende Tests, wie die Reizschwellenbestimmung und die Impedanztestung durchgeführt wurden. Die Stimulationsausgabe (Pacing output) wurde auf das mindestens Doppelte der gemessenen Reizschwelle programmiert. Die ICDs wurden je nach kardialer Grunderkrankung mit verschiedenen Detektionszonen programmiert. So gab es beispielsweise bei der antitachykarden Therapie eine ventrikuläre Tachykardie (VT) Zone mit einem Therapiebereich ab 170 Schlägen/min. In diesem Bereich soll das Gerät mehrmalige Versuche von antitachykarder Stimulation (ATP) abgeben (Burst- und Ramp-Stimulation) bevor ein Schock abgegeben wird. Des Weiteren gab es eine ventrikuläre Fibrillations (VF) Zone ab 220 Schlägen/min (für 1218 aus 16-24 Schlägen), ab der der ICD während der Ladephase antitachykarde Stimulation gefolgt von einem Schock maximaler Energie (30-35 J) abgeben sollte. In der Sekundärprophylaxe orientierte sich die ICD-Programmierung an den vorangegangenen tachykarden Rhythmusstörungen. In der antibradykarden Therapie wurde bei VVI-ICDs eine Interventionsfrequenz von 40 Schlägen/min gewählt. Bei DDD- und CRT-D-Geräten wurde eine Interventionsfrequenz zwischen 60 und 130 Schlägen/min eingestellt. Die Interventionsfrequenzen und Modi wurden ebenfalls an die individuellen Patientenbedürfnisse angepasst. Um inappropriate Schocks zu verhindern wurden in neueren Geräten sogenannte Onset- und Stability-Kriterien aktiviert (Biotronik „S.M.A.R.T.“; Medtronic „Wavelet“ / „PRLogic“; Boston Scientific „RhythmID“).

\subsection{Datenanalyse}

Die Datenanalyse erfolgte mit dem Statistikprogramm „IBM® SPSS® Statistics version 20". Die deskriptive Statistik zur Beschreibung der patientenbezogenen Daten umfasst überwiegend Mittelwerte und die zugehörigen Standardabweichungen. Die 
Batterielaufzeitanalysen wurden mit Hilfe der Kaplan-Meier-Methode berechnet. Patienten, die vor der Wechseloperation verstarben oder das Follow-Up-Datum erreichten, wurden zensiert. Um statistische Vergleiche verschiedener Gruppen, wie beispielsweise den verschiedenen ICD-Herstellern, den ICD-Typen, dem prozentualen Stimulationsanteil und der Anzahl an Schocks durchzuführen, wurde der Log-RankTest angewendet. Hierbei wurde eine Irrtumswahrscheinlichkeit von $p<0,05$ als statistisch signifikant betrachtet. Um den Einfluss verschiedener Parameter auf das relative Risiko einer ICD-Wechseloperation zu errechnen wurden univariate und multivariate Cox-Regressionsanalysen durchgeführt. Die Ergebnisse wurden als Hazard Ratio mit dem dazugehörigen $95 \%$ Konfidenzintervall angegeben. Die multivariaten Analysen wurden mit Hilfe des Wald-Tests und Rückwärtselimination durchgeführt. Variablen, die in der univariaten Analyse einen p-Wert von unter 0,1 erreichten, wurden in die multivariate Analyse eingeschlossen. Auch hier wurde eine Irrtumswahrscheinlichkeit von $p<0,05$ als statistisch signifikant betrachtet.

\subsection{Datenschutz}

Sämtliche Patientendaten wurden anonymisiert in die Datenbank aufgenommen. Eine Zuordnung der Personen ist dadurch nachträglich nicht mehr möglich. 


\section{Ergebnisse}

\subsection{Deskriptive Statistik}

\subsubsection{Patientencharakteristika}

In diese Analyse wurden 1698 Patienten eingeschlossen. Allen wurde im Zeitraum von März 1987 bis Juni 2012 ein ICD an der Universitätsmedizin Göttingen implantiert. Größtenteils (93,2 \%) wurden sie auch hier nachgesorgt. Das mittlere Alter der Patienten bei Erstimplantation betrug 63,0 \pm 13,2 Jahre. Das Geschlechterverhältnis lag bei 1375 (81\%) männlichen Patienten zu 323 (19\%) weiblichen Patienten. Während der Nachsorge, die im Mittel 5,9 \pm 3,6 Jahre dauerte, verstarben 644 (37,9 \%) Patienten.

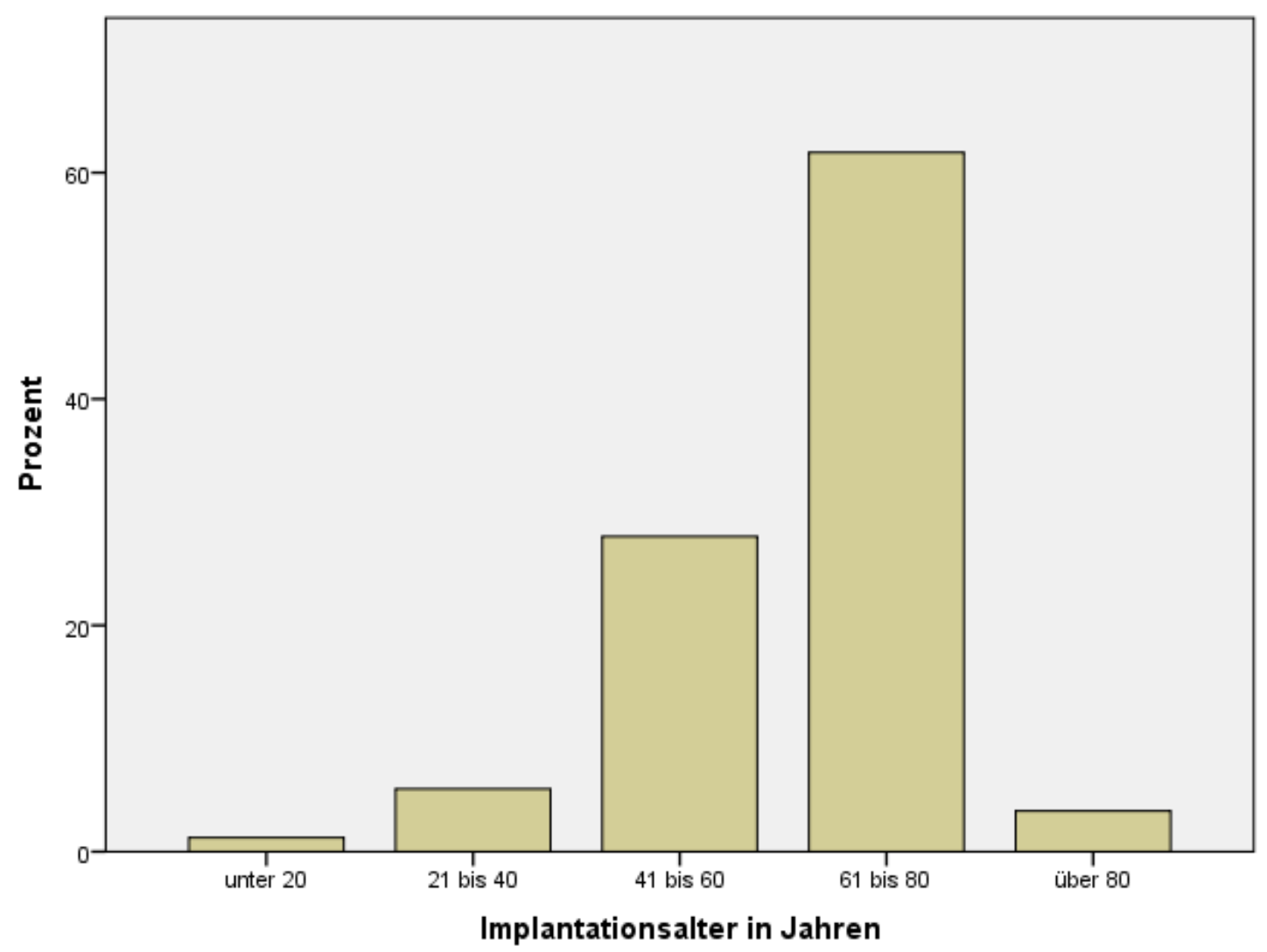

Abbildung 1: Altersverteilung bei Erstimplantation 


\subsubsection{Kardiale Grunderkrankungen}

Die meisten Patienten (65\%) litten an einer KHK und hatten im Durchschnitt bei Erstimplantation eine verminderte linksventrikuläre Ejektionsfraktion von 30,8 \pm 12,8 \%. Die zweithäufigste Grunderkrankung (32,9\%) war eine DCM. Tabelle 1 zeigt die Mittelwerte und Standardabweichungen für klinische Patientencharakteristika sowie für kardiale Grunderkrankungen des Patientenkollektivs.

Tabelle 1: Patientencharakteristika und kardiale Grunderkrankungen

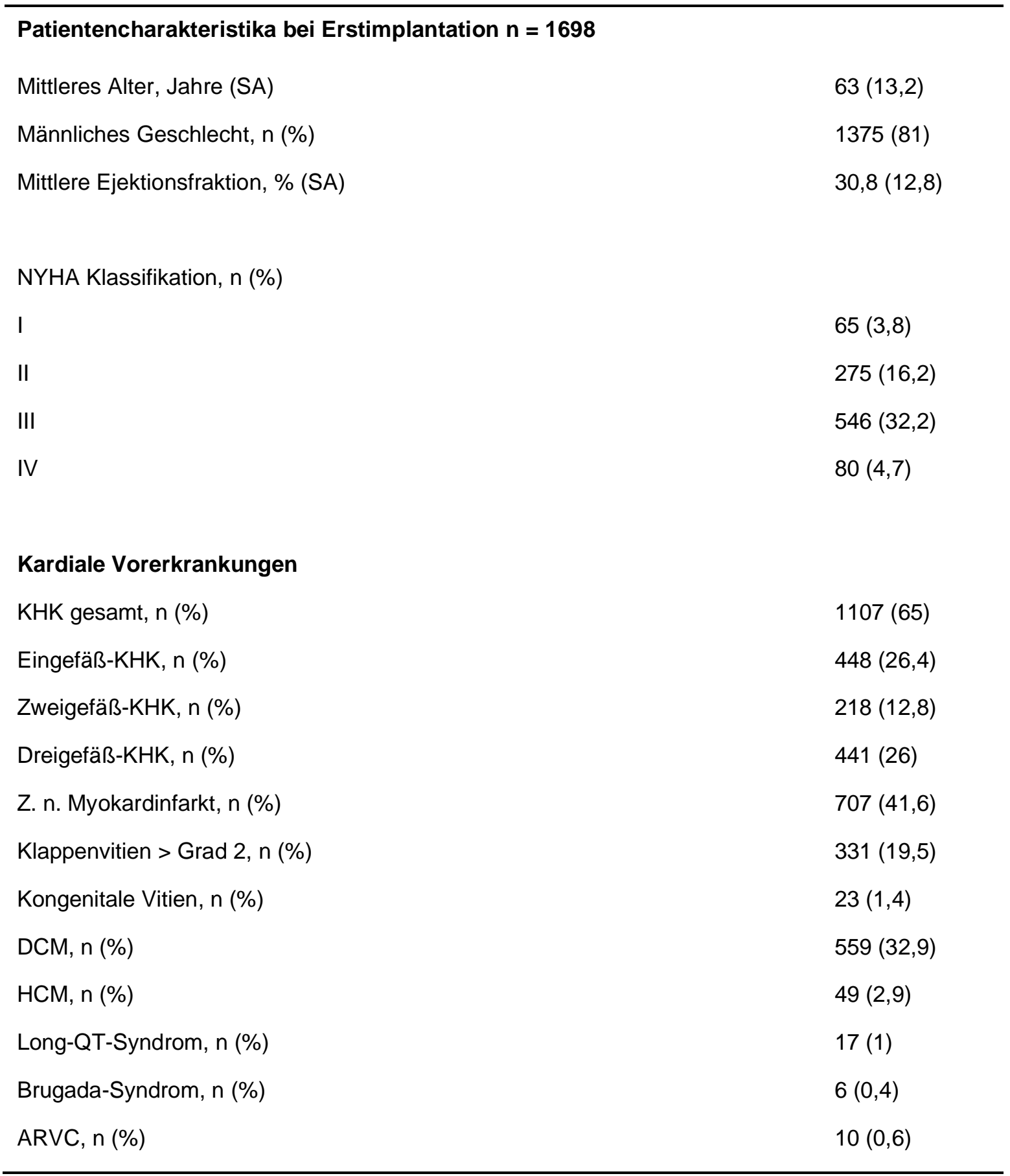




\subsubsection{ICD-Indikation}

Die Indikation zur ICD-Implantation richtete sich stets nach den jeweils aktuellen Leitlinien. Die Leitlinien unterliegen auf Grund medizinischer Fortschritte und neuen Erkenntnissen einem stetigen Wandel. Bei dem untersuchten Patientenkollektiv wurde 805 (47,4\%) Patienten ein ICD primärprophylaktisch implantiert; 893 (52,6 \%) Patienten bekamen einen ICD nach einem kardialen Ereignis sekundärprophylaktisch implantiert, wobei mehrere Gründe parallel vorliegen konnten (Tabelle 2).

Tabelle 2: ICD-Indikation und kardiale Ereignisse vor Erstimplantation

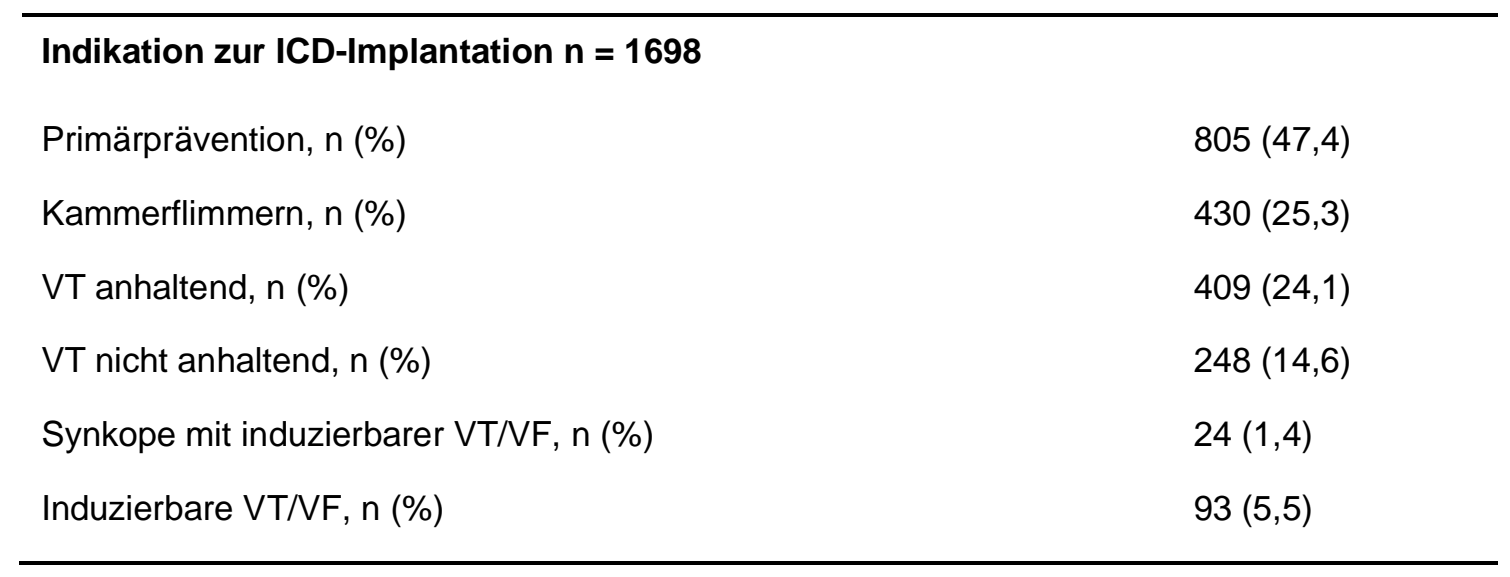

\subsubsection{Kardiale Medikation bei Erstimplantation}

Die kardiale Medikation des Patientenkollektivs wurde stets nach den jeweils aktuellen Leitlinien vorgenommen, gegebenenfalls individuell angepasst. Beispielsweise nahmen bei Erstimplantation eines ICDs 1170 (68,9 \%) Patienten einen Betablocker. Hierbei lagen sowohl Kombinationen mehrerer Medikamente als auch Monotherapien vor. Die genaue Verteilung zeigt Tabelle 3. 
Tabelle 3: Kardiale Medikation bei ICD-Erstimplantation

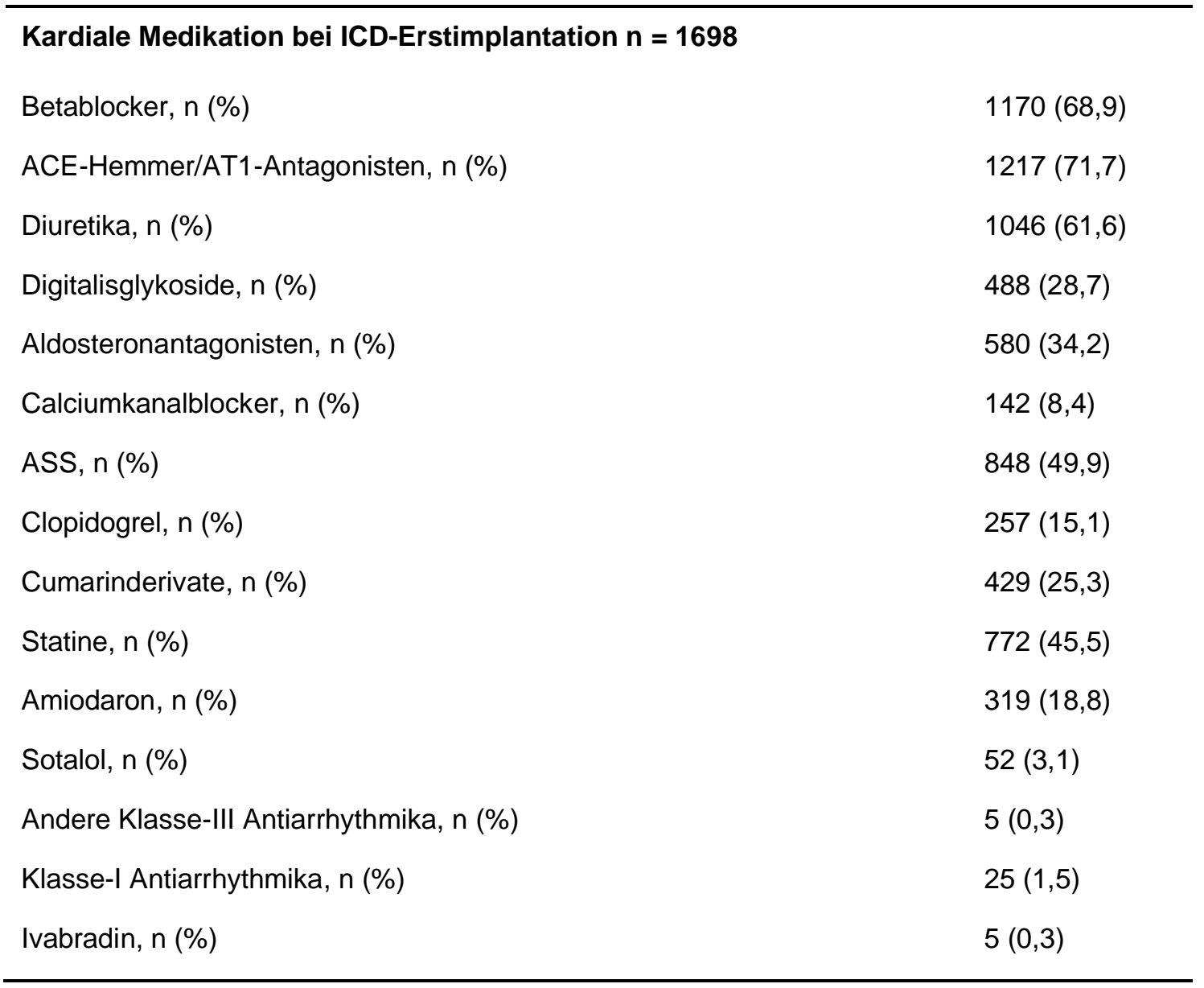

\subsubsection{Implantierte Defibrillatoren}

Bei 1698 Patienten wurden insgesamt 2408 ICDs implantiert. Es gab 710 Wechseloperationen. Hiervon waren 587 (82,7 \%) durch Batterieerschöpfung (ERI) bedingt und 123 (17,3\%) wurden aus anderen Gründen, wie beispielsweise einem System-Upgrade durchgeführt. Die Gründe für Wechseloperationen sind in Tabelle 4 dargestellt. Von den 587 durch ERI bedingten Aggregatwechseln lagen bei 46 Geräten zusätzliche Gründe für eine Wechseloperation vor. Eine Batterieerschöpfung war hier jedoch immer gegeben. Bei 19 Aggregatwechseln war der Grund retrospektiv nicht zu erheben und bei 36 nicht batteriebedingten Wechseloperationen lagen mehrere Gründe vor. Von 1698 Patienten bekamen während der Nachsorge 518 (21,5\%) Patienten zwei ICDs implantiert. 149 (6,2\%) Patienten bekamen drei ICDs, 34 (1,4 \%) Patienten bekamen vier ICDs und acht $(0,3 \%)$ Patienten bekamen fünf ICDs implantiert. Ein Patient bekam insgesamt sechs ICDs implantiert. Die implantierten ICD-Typen und Hersteller sind in Tabelle 5 zu sehen. 
Tabelle 4: Gründe für Wechseloperationen

\begin{tabular}{lll}
\hline Wechselgrund & Anzahl & Überschneidungen mit ERI \\
Batterieerschöpfung (ERI) & $587(82,7 \%)$ & $/$ \\
System-Upgrade & $114(16 \%)$ & $40(5,6 \%)$ \\
Elektronische-Komponenten & $32(4,5 \%)$ & $3(<1 \%)$ \\
Konnektor & $7(1 \%)$ & \\
Sensor & $10(1,4 \%)$ & $2(<1 \%)$ \\
Software & $6(<1 \%)$ & $1(<1 \%)$ \\
Infektion & $17(2,4 \%)$ & \\
Unbekannt & $19(2,7 \%)$ & $46(6,5 \%)$ \\
Gesamt & $710(100 \%)$ & \\
\hline
\end{tabular}


Tabelle 5: Implantierte Defibrillatoren

\begin{tabular}{|c|c|c|c|c|c|c|c|c|c|c|}
\hline VVI & $\mathrm{n}$ & ERI & DDD & $\mathrm{n}$ & ERI & CRT-D & $\mathrm{n}$ & ERI & Gesamt $n$ & ERI \\
\hline Gesamt & 1050 & 284 & & 651 & 155 & & 707 & 148 & 2408 & 587 \\
\hline Boston Scientific: & 570 & 190 & & 186 & 54 & & 287 & 51 & 1043 & 295 \\
\hline Teligen VR & 2 & 0 & Punctua DR & 2 & 0 & Cognis & 4 & 0 & & \\
\hline Ventak & 6 & 4 & Teligen DR & 5 & 0 & Contak CD & 4 & 3 & & \\
\hline Ventak AV & 2 & 1 & Ventak AV II DR & 11 & 3 & Contak Renewal & 18 & 6 & & \\
\hline Ventak Mini & 4 & 2 & Ventak AV III DR & 6 & 2 & Contak Renewal 2 & 27 & 11 & & \\
\hline Ventak Mini HC & 6 & 4 & Ventak PRIZM 2 DR & 30 & 14 & Contak Renewal 4 & 56 & 16 & & \\
\hline Ventak Mini II & 1 & 0 & Ventak PRIZM DR & 15 & 5 & Contak Renewal 4 AVT & 8 & 4 & & \\
\hline Ventak Mini II+ & 37 & 20 & Vitality 2 DR & 74 & 11 & Contak Renewal $4 \mathrm{HE}$ & 2 & 1 & & \\
\hline Ventak Mini III+ & 20 & 5 & Vitality 2 DR EL & 11 & 3 & Contak Renewal 4 RF & 108 & 10 & & \\
\hline Ventak Mini IV+ & 31 & 17 & Vitality AVT & 1 & 1 & Livian & 59 & 0 & & \\
\hline Ventak Mini+ & 26 & 10 & Vitality DR & 30 & 14 & Punctua CRT-D & 1 & 0 & & \\
\hline Ventak Mini+ HC & 20 & 9 & Vitality DR+ & 1 & 1 & & & & & \\
\hline Ventak P & 19 & 10 & & & & & & & & \\
\hline Ventak P2 & 26 & 13 & & & & & & & & \\
\hline Ventak P3 & 2 & 2 & & & & & & & & \\
\hline Ventak Prizm 2 VR & 48 & 6 & & & & & & & & \\
\hline Ventak Prizm VR & 19 & 7 & & & & & & & & \\
\hline Ventak Prizm VR HE & 7 & 4 & & & & & & & & \\
\hline Ventak PRx & 29 & 17 & & & & & & & & \\
\hline Ventak PRx II & 36 & 16 & & & & & & & & \\
\hline Ventak PRx III & 24 & 12 & & & & & & & & \\
\hline Ventak PRx III HC & 10 & 5 & & & & & & & & \\
\hline Ventak VR & 4 & 1 & & & & & & & & \\
\hline Vitality 2 VR & 130 & 7 & & & & & & & & \\
\hline Vitality 2 VR EL & 24 & 3 & & & & & & & & \\
\hline Vitality VR & 37 & 15 & & & & & & & & \\
\hline
\end{tabular}


Tabelle 5: Implantierte Defibrillatoren

\begin{tabular}{|c|c|c|c|c|c|c|c|c|c|c|}
\hline VVI & $\mathrm{n}$ & ERI & DDD & $\mathrm{n}$ & $\mathrm{ERI}$ & CRT-D & $\mathrm{n}$ & ERI & Gesamt n & ER \\
\hline Medtronic: & 366 & 89 & & 381 & 91 & & 412 & 97 & 1159 & 277 \\
\hline Egida VR & 3 & 0 & Egida DR & 5 & 0 & Concerto & 139 & 21 & & \\
\hline Entrust Escudo & 1 & 0 & Entrust ATG & 67 & 14 & Consulta & 17 & 2 & & \\
\hline Entrust VR & 58 & 0 & GEM DR & 14 & 7 & Egida-CRT & 13 & 0 & & \\
\hline GEM & 27 & 11 & GEM II DR & 9 & 6 & InSync ICD & 6 & 4 & & \\
\hline GEM ॥ VR & 11 & 6 & GEM III DR & 30 & 19 & InSync III Marquis & 98 & 25 & & \\
\hline GEM III VR & 3 & 3 & GEM III AT & 14 & 10 & InSync Marquis & 4 & 1 & & \\
\hline GEM VR & 9 & 5 & Intrinsic & 53 & 12 & InSync Maximo & 14 & 2 & & \\
\hline Jewel PCD & 15 & 9 & Jewel AF & 4 & 3 & InSync Sentry & 116 & 42 & & \\
\hline Jewel Plus & 8 & 5 & Marquis DR & 59 & 16 & Maximo II & 2 & 0 & & \\
\hline Marquis VR & 147 & 22 & Maximo DR & 2 & 0 & Protecta CRT-D & 1 & 0 & & \\
\hline Maximo VR & 12 & 0 & Maximo II DR & 2 & 0 & Protecta XT CRT-D & 2 & 0 & & \\
\hline Maximo II VR & 1 & 0 & Secura DR & 18 & 0 & & & & & \\
\hline Micro Jewel & 7 & 4 & Virtuoso DR & 104 & 4 & & & & & \\
\hline Micro Jewel II & 35 & 19 & & & & & & & & \\
\hline PCD & 3 & 3 & & & & & & & & \\
\hline Virtuoso VR & 26 & 2 & & & & & & & & \\
\hline Biotronik: & 113 & 4 & & 84 & 10 & & 6 & 0 & 203 & 14 \\
\hline Lexos VR & 30 & 0 & Lexos $A_{+} / T$ & 15 & 6 & Lumax $300 \mathrm{HF}-\mathrm{T}$ & 6 & 0 & & \\
\hline Lexos VR-T & 3 & 0 & Lexos DR & 16 & 0 & & & & & \\
\hline Lumax 300 VR-T & 63 & 3 & Lexos DR-T & 5 & 0 & & & & & \\
\hline Lumax 340 VR-T & 2 & 0 & Lumax 300 DR-T & 26 & 0 & & & & & \\
\hline Lumos VR-T & 15 & 1 & Lumos DR-T & 22 & 4 & & & & & \\
\hline St. Jude Medical: & 1 & 1 & & l & l & & 2 & 0 & 3 & 1 \\
\hline \multirow[t]{2}{*}{ Cadet } & 1 & 1 & & & & Atlas DR V-243 & 1 & 0 & & \\
\hline & & & & & & Atlas DR V-268 & 1 & 0 & & \\
\hline
\end{tabular}




\subsection{Batterielaufzeitanalysen}

\subsubsection{Einfluss des ICD-Herstellers auf die Batterielaufzeit}

Um den Einfluss des Herstellers auf die ICD-Batterielaufzeit zu beschreiben, wurden 2405 ICDs untersucht. Aggregate des Herstellers St. Jude Medical wurden hier auf Grund der geringen Anzahl nicht berücksichtigt. Nur batteriebedingte Aggregatwechsel wurden als Ereignis definiert. In Tabelle 6 sind die funktionellen Eigenschaften der untersuchten ICDs (Stimulationsanteil und abgegebene Schocks) nach Herstellern unterteilt dargestellt. Die ICDs der drei untersuchten Hersteller zeigten dabei unterschiedliche Batterielaufzeiten (Abbildung 2). Medtronic-ICDs hielten signifikant länger als Boston-Scientific-ICDs $(p<0,001)$. Auf Grund der geringeren Anzahl an Biotronik-ICDs ist der direkte Vergleich aller Kammertypen zwischen Biotronik und Medtronic / Boston Scientific statistisch nicht signifikant (Tabelle 7). Medtronic-ICDs zeigten eine mediane Batterielaufzeit von 6,9 Jahren (95\% Konfidenzintervall (KI), 6,7-7,2 Jahre). Geräte der Firma Boston Scientific / Guidant wurden im Median nach 5,8 Jahren (95\% Kl, 5,6-6 Jahre) ausgetauscht und bei ICDs der Firma Biotronik wurden batteriebedingte Wechseloperationen nach im Median 5,2 Jahren (95\% KI, 5,1-5,4 Jahre) durchgeführt.

Tabelle 6: Eigenschaften der ICDs zum Einfluss des Herstellers auf die Batterielaufzeit

\begin{tabular}{llll}
\hline Variable & BSI $(\mathbf{n}=\mathbf{1 0 4 3})$ & MED $(\mathbf{n}=\mathbf{1 1 5 9})$ & BIO (n= 203) \\
$\begin{array}{llll}\text { Anzahl Schocks } \\
\text { Kein Schock }\end{array}$ & & & \\
1 & $476(45,6 \%)$ & $602(51,9 \%)$ & $130(64 \%)$ \\
$2-4$ & $114(10,9 \%)$ & $105(9 \%)$ & $19(9,4 \%)$ \\
5 oder mehr & $97(9,3 \%)$ & $97(8,4 \%)$ & $10(4,9 \%)$ \\
& $203(19,5 \%)$ & $163(14 \%)$ & $9(4,4 \%)$ \\
Stimulationsanteil & & & $104(51,2 \%)$ \\
$0-50 \%$ & $428(41 \%)$ & $397(34,3 \%)$ & $21(10,3 \%)$ \\
$51-150 \%$ & $81(7,8 \%)$ & $154(13,3 \%)$ & $14(6,9 \%)$ \\
$151-300 \%$ & $292(28 \%)$ & $413(35,6 \%)$ & \\
\hline
\end{tabular}




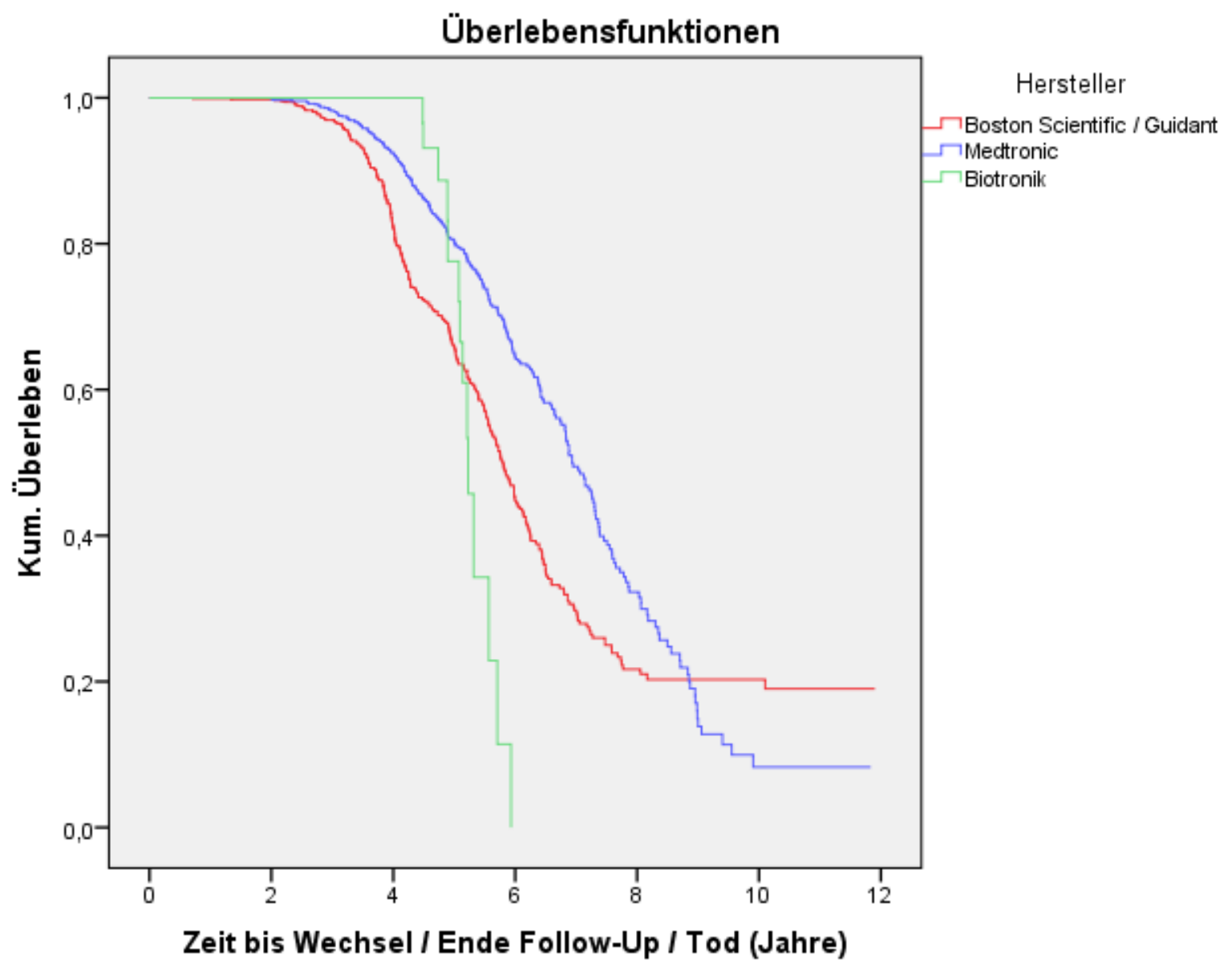

\begin{tabular}{|c|c|c|c|c|c|c|c|}
\hline 1043 & 781 & 397 & 127 & 33 & 16 & 0 & BSI \\
\hline 1159 & 914 & 605 & 209 & 44 & 5 & 0 & MED \\
\hline 203 & 130 & 43 & 0 & & & & BIO \\
\hline
\end{tabular}

Abbildung 2: Einfluss des Herstellers auf die Batterielaufzeit

Tabelle 7: Einfluss des Herstellers auf die Batterielaufzeit

\begin{tabular}{llll}
\hline Log-Rank-Test & Chi-Quadrat & Freiheitsgrade & Signifikanz (p-Wert) \\
Gesamtvergleich & 29,904 & 2 & $<0,001$ \\
BSI vs. MED & 29,521 & 1 & $<0,001$ \\
BSI vs. BIO & 1,178 & 1 & 0,278 \\
MED vs. BIO & 2,283 & 1 & 0,131 \\
\hline
\end{tabular}

Zur weiteren Differenzierung bezüglich des Einflusses der ICD-Hersteller auf die Batterielaufzeit wurden die verschiedenen Kammertypen separat auf herstellerbedingte Unterschiede untersucht. Nur die VVI-Geräte betrachtet, zeigt sich ein noch deutlicherer, signifikanter Unterschied hinsichtlich der Batterielaufzeit der Hersteller Medtronic und Boston Scientific / Guidant (Abbildung 3, Tabelle 8). 
Medtronic-VVIs zeigten eine mediane Batterielaufzeit von 7,9 Jahren (95 \% KI, 7,38,4 Jahre). VVI-Geräte der Firma Boston Scientific / Guidant wurden im Median nach 5,8 Jahren (95 \% KI, 5,5-6,2 Jahre) ausgetauscht und bei VVIs der Firma Biotronik wurden batteriebedingte Wechseloperationen nach im Median 4,7 Jahren (95\% KI, 4,4-5,1 Jahre) durchgeführt.

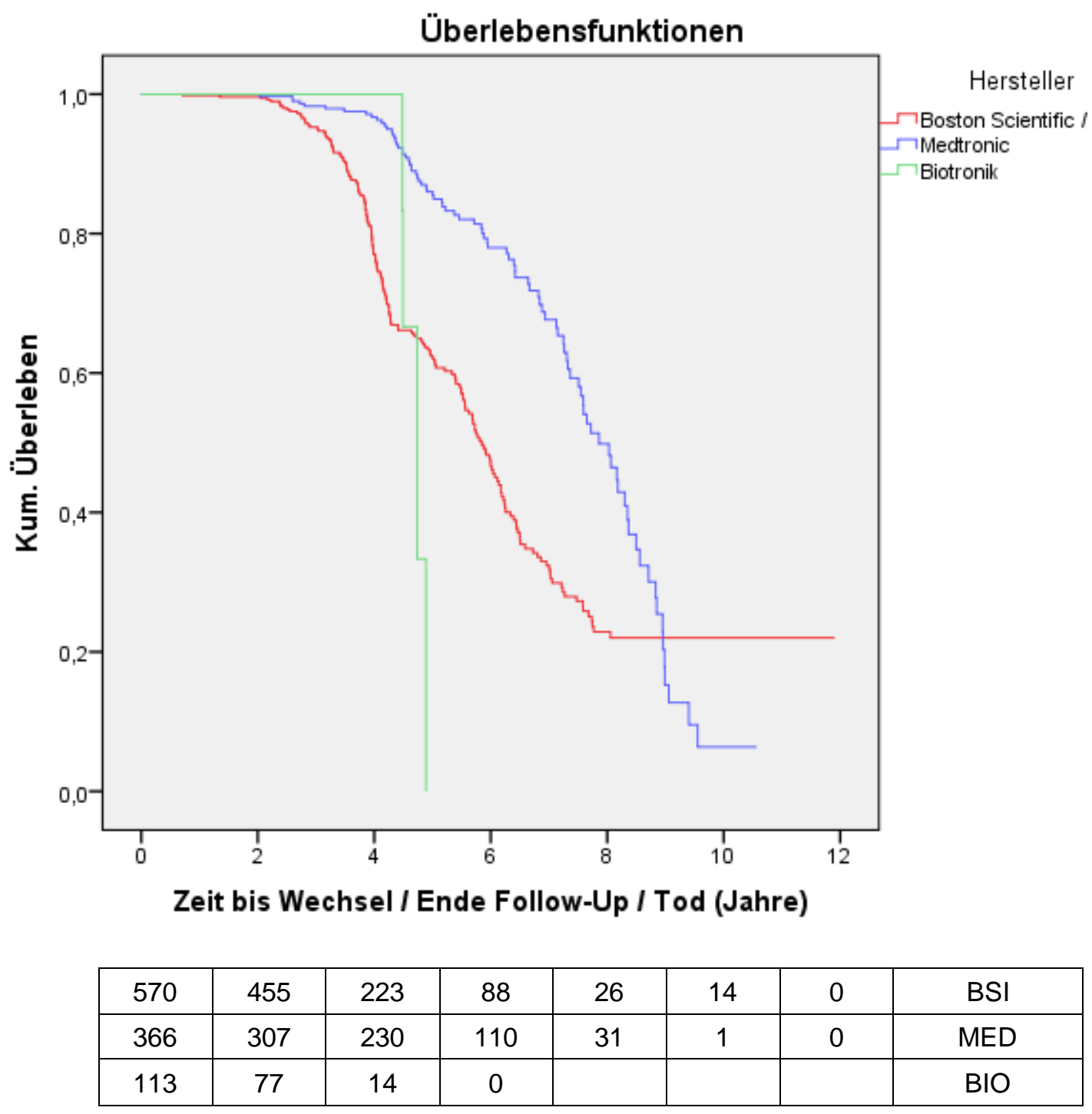

Abbildung 3: Einfluss des Herstellers auf die VVI-Batterielaufzeit

Tabelle 8: Einfluss des Herstellers auf die VVI-Batterielaufzeit

\begin{tabular}{llll}
\hline Log-Rank-Test & Chi-Quadrat & Freiheitsgrade & Signifikanz (p-Wert) \\
Gesamtvergleich & 38,595 & 2 & $<0,001$ \\
BSI vs. MED & 37,638 & 1 & $<0,001$ \\
BSI vs. BIO & 2,287 & 1 & 0,13 \\
MED vs. BIO & 3,025 & 1 & 0,082 \\
\hline
\end{tabular}


In der separaten Analyse der DDD-Geräte zeigt sich, zusätzlich zu den bereits in der Analyse aller Kammertypen gesehenen signifikanten Herstellerunterschieden, ein signifikanter Unterschied der Batterielaufzeiten von Biotronik-DDDs und MedtronicDDDs (Abbildung 4, Tabelle 9). Für DDDs des Herstellers Medtronic konnte eine mediane Batterielaufzeit von 6,8 Jahren (95\% KI, 6,6-7 Jahre), für Biotronik-DDDs von 5,3 Jahren (95 \% KI, 5,1-5,5 Jahre) und für Boston-Scientific- / Guidant-DDDs von 5,8 Jahren (95\% KI, 5,4-6,1 Jahre) ermittelt werden.

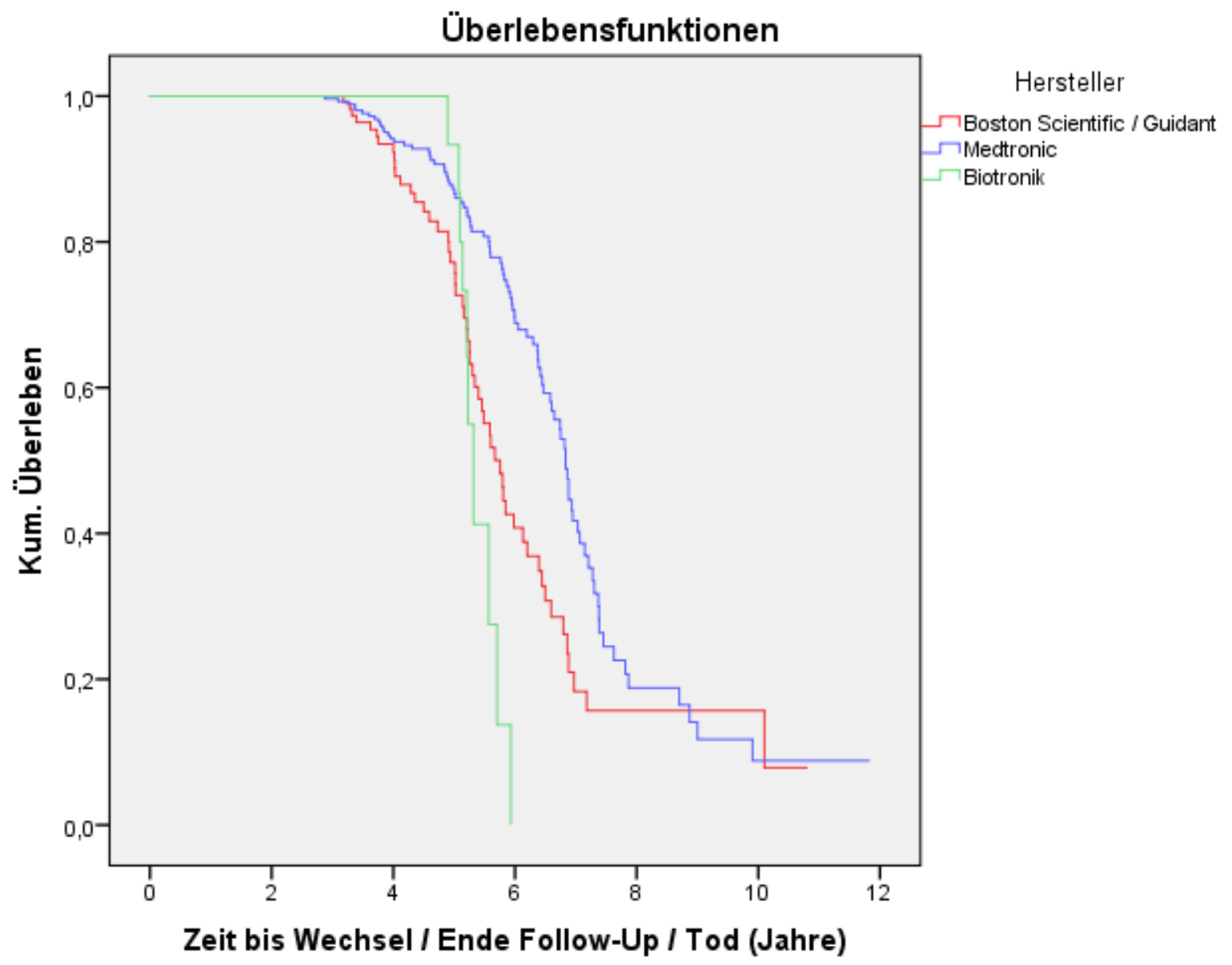

\begin{tabular}{|c|c|c|c|c|c|c|c|}
\hline 186 & 143 & 84 & 22 & 5 & 2 & 0 & BSI \\
\hline 381 & 313 & 210 & 78 & 9 & 3 & 0 & MED \\
\hline 84 & 53 & 29 & 0 & & & & BIO \\
\hline
\end{tabular}

Abbildung 4: Einfluss des Herstellers auf die DDD-Batterielaufzeit 
Tabelle 9: Einfluss des Herstellers auf die DDD-Batterielaufzeit

\begin{tabular}{llll}
\hline Log-Rank-Test & Chi-Quadrat & Freiheitsgrade & Signifikanz (p-Wert) \\
Gesamtvergleich & 15,176 & 2 & $\mathbf{0 , 0 0 1}$ \\
BSI vs. MED & 11,312 & 1 & $\mathbf{0 , 0 0 1}$ \\
BSI vs. BIO & 0,254 & 1 & 0,614 \\
MED vs. BIO & 8,266 & 1 & $\mathbf{0 , 0 0 4}$ \\
\hline
\end{tabular}

Werden nur die CRT-D-Geräte untersucht, so kann in dieser Analyse kein signifikanter Unterschied der Batterielaufzeiten von Medtronic-CRT-Ds (Median 5,6 Jahre $(95 \% \mathrm{KI}$, 5,4-5,7 Jahre)) und Boston-Scientific- / Guidant-CRT-Ds (Median 5,8 Jahre (95 \% KI, 5,3-6,2 Jahre)) gezeigt werden (Abbildung 5, Tabelle 10).

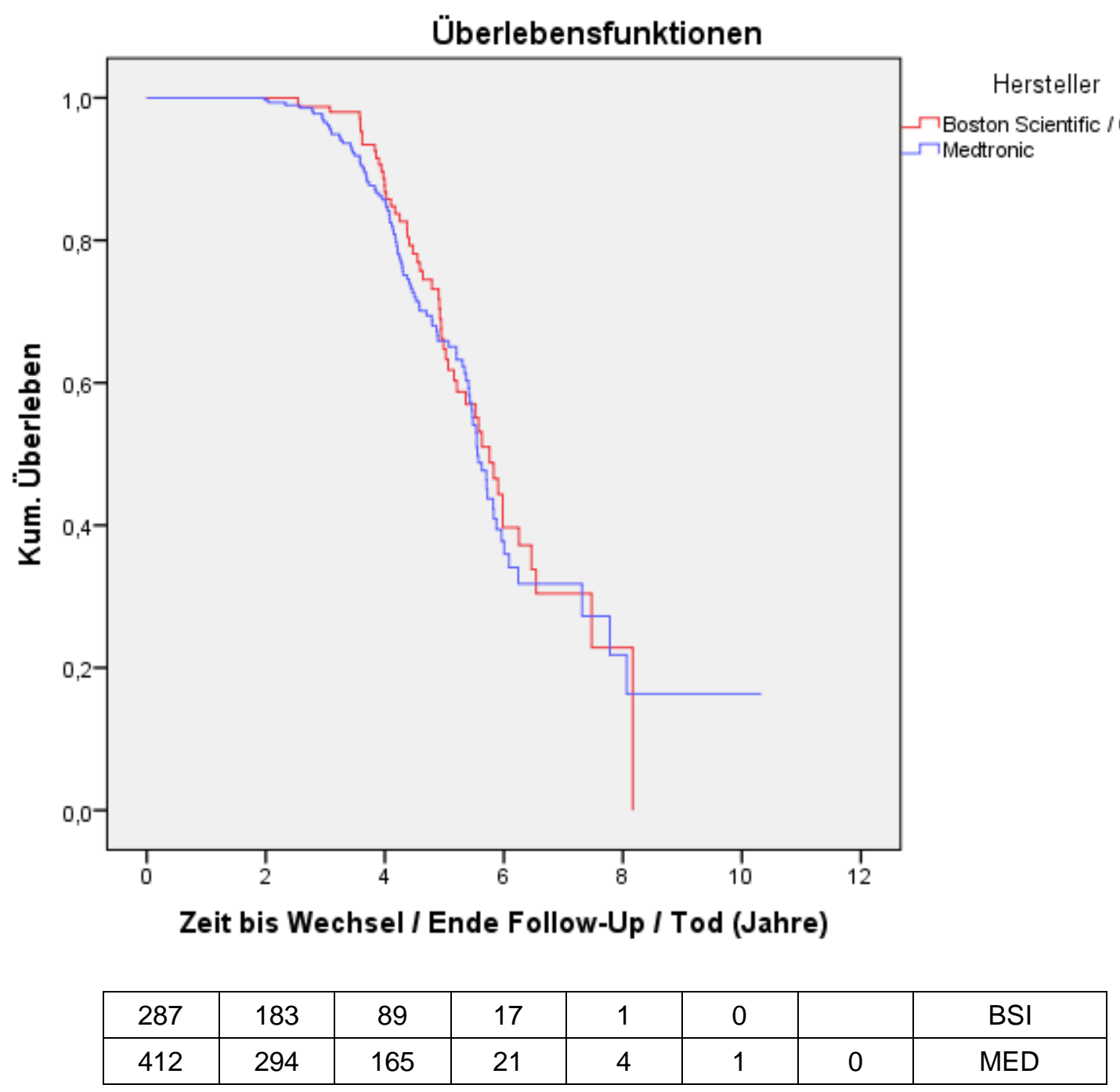

Abbildung 5: Einfluss des Herstellers auf die CRT-D-Batterielaufzeit 
Tabelle 10: Einfluss des Herstellers auf die CRT-D-Batterielaufzeit

\begin{tabular}{llll}
\hline Log-Rank-Test & Chi-Quadrat & Freiheitsgrade & Signifikanz (p-Wert) \\
BSI vs. MED & 0,363 & 1 & 0,547 \\
\hline
\end{tabular}

\subsubsection{Einfluss des ICD-Typs auf die Batterielaufzeit}

Für die verschiedenen ICD-Typen (VVI-, DDD-ICD, CRT-D) konnten signifikant unterschiedliche Batterielaufzeiten ermittelt werden (Tabelle 12). Die 2408 nach der Kaplan-Meier-Methode analysierten ICDs (Abbildung 6) zeigten dabei unterschiedliche Eigenschaften hinsichtlich des Stimulationsanteils und der abgegebenen Schocks (Tabelle 11). So hatten beispielsweise CRT-Ds im Gegensatz zu VVIs einen hohen prozentualen Stimulationsanteil. Die relativ am meisten abgegebenen Schocks wurden von VVI-Geräten ausgelöst. So gaben 22,2 \% der untersuchten VVI-Geräte im Gegensatz zu 8,8 \% der CRT-D-Geräte multiple (5 oder mehr) Schocks ab. VVI-Geräte zeigten mit im Median 6,8 Jahren (95 \% KI, 6,4-7,2 Jahre) die längsten Batterielaufzeiten, gefolgt von DDD-Geräten mit einer medianen Batterielaufzeit von 6,5 Jahren (95\% KI, 6,2-6,8 Jahre). Die signifikant gegenüber den anderen Kammertypen kürzeste mediane Batterielaufzeit zeigten CRT-Ds mit 5,6 Jahren (95\% KI, 5,4-5,9 Jahre).

Tabelle 11: Eigenschaften der ICDs zum Einfluss des Kammertyps auf die Batterielaufzeit

\begin{tabular}{|c|c|c|c|}
\hline Variable & VVI $(n=1050)$ & $\operatorname{DDD}(n=651)$ & CRT-D (n = 707) \\
\hline \multicolumn{4}{|c|}{ Anzahl Schocks } \\
\hline Kein Schock & 435 (41,4 \%) & 339 (52,1%) & $434(61,4 \%)$ \\
\hline 1 & $115(11 \%)$ & $71(11 \%)$ & $53(7,5 \%)$ \\
\hline $2-4$ & $98(9,3 \%)$ & $58(5,5 \%)$ & 48 (6,8 \%) \\
\hline 5 oder mehr & $233(22,2 \%)$ & $80(12,3 \%)$ & $62(8,8 \%)$ \\
\hline \multicolumn{4}{|c|}{ Stimulationsanteil } \\
\hline $0-50 \%$ & $621(59,1 \%)$ & $271(41,6 \%)$ & 38 (5,4%) \\
\hline $51-150 \%$ & $63(6 \%)$ & $156(24 \%)$ & $37(5,2 \%)$ \\
\hline $151-300 \%$ & $60(6,7 \%)$ & $101(15,5 \%)$ & $560(79,2 \%)$ \\
\hline
\end{tabular}




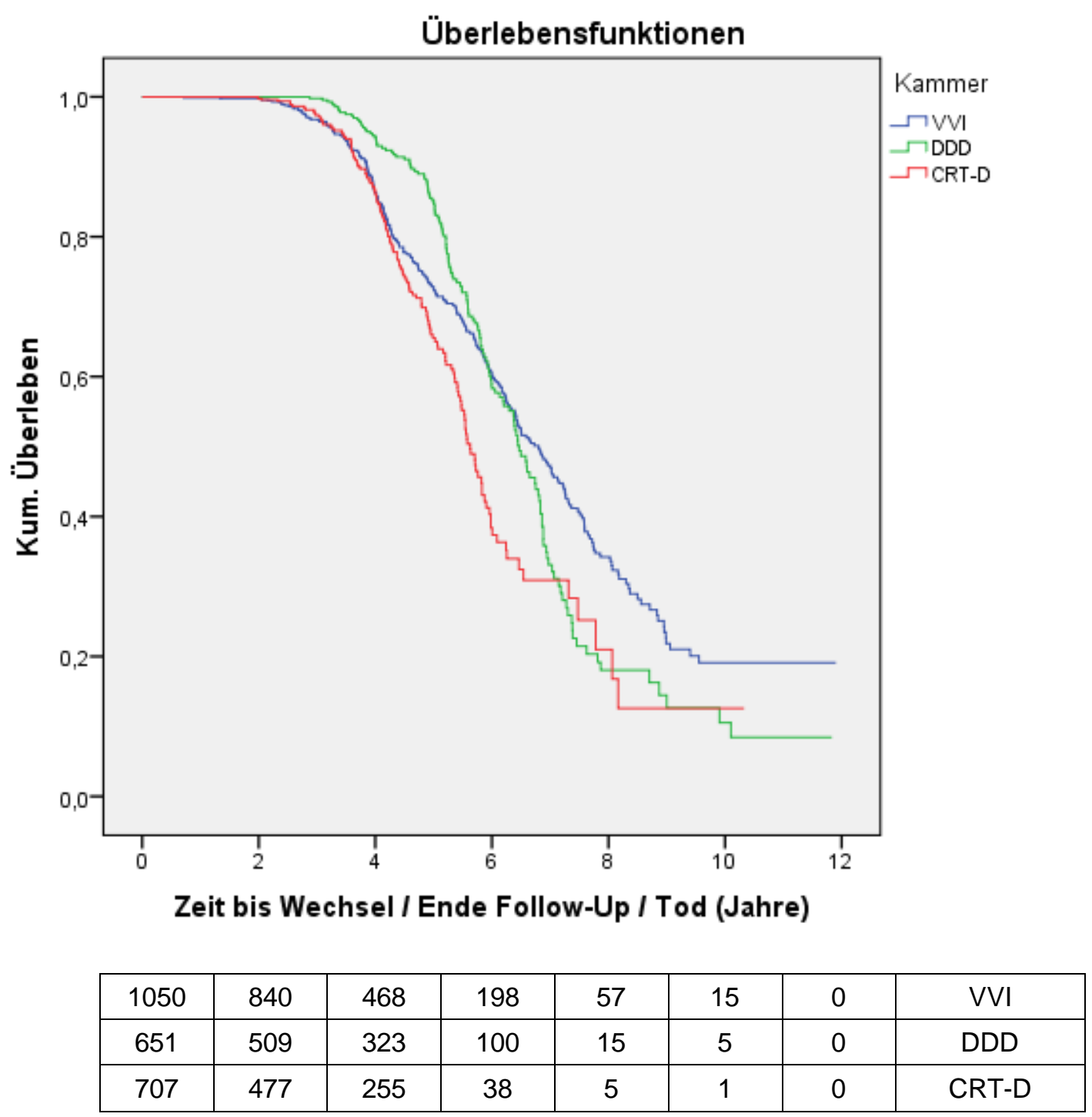

Abbildung 6: Einfluss des Kammertyps auf die Batterielaufzeit

Tabelle 12: Einfluss des Kammertyps auf die Batterielaufzeit

\begin{tabular}{llll}
\hline Log-Rank-Test & Chi-Quadrat & Freiheitsgrade & Signifikanz (p-Wert) \\
Gesamtvergleich & 20,896 & 2 & $<\mathbf{0 , 0 0 1}$ \\
VVI vs. DDD & 0,007 & 1 & 0,931 \\
VVI vs. CRT-D & 12,262 & 1 & $<\mathbf{0 , 0 0 1}$ \\
DDD vs. CRT-D & 19,117 & 1 & $<\mathbf{0 , 0 0 1}$ \\
\hline
\end{tabular}

\subsubsection{Einfluss der Stimulation auf die Batterielaufzeit}

Von 1907 ICDs lagen Daten über den prozentualen Stimulationsanteil vor, 521 dieser ICDs wurden auf Grund von Batterieerschöpfung ausgetauscht. Die Kaplan-MeierKurve (Abbildung 7) zeigt einen signifikanten Einfluss (Tabelle 13) der prozentualen 
Stimulation auf die ICD-Batterielaufzeit. Geräte, die einen hohen Stimulationsanteil hatten (151-300 \%), zeigten signifikant kürzere Batterielaufzeiten als Geräte, die einen niedrigen Stimulationsanteil $(0-50 \%)(p<0,001)$ bzw. Geräte die einen mittleren Stimulationsanteil $(51-150 \%)(p=0,009)$ hatten.

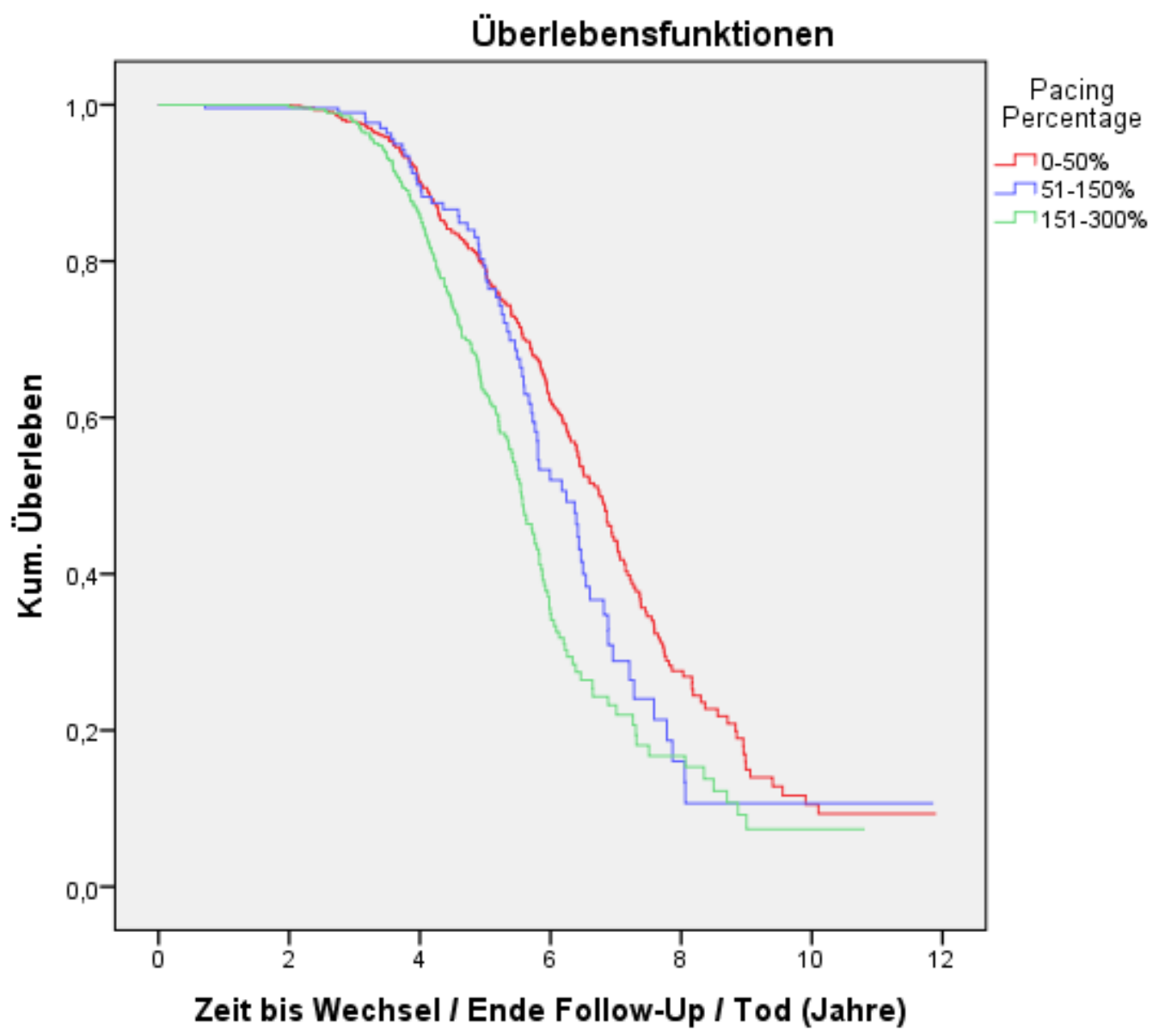

\begin{tabular}{|c|c|c|c|c|c|c|c|}
\hline 930 & 766 & 457 & 184 & 39 & 9 & 0 & $0-50 \%$ \\
\hline 256 & 191 & 118 & 39 & 6 & 1 & 0 & $51-150 \%$ \\
\hline 721 & 516 & 284 & 46 & 12 & 2 & 0 & $151-300 \%$ \\
\hline
\end{tabular}

Abbildung 7: Einfluss der Stimulation auf die Batterielaufzeit

Tabelle 13: Einfluss der Stimulation auf die Batterielaufzeit

\begin{tabular}{llll}
\hline Log-Rank-Test & Chi-Quadrat & Freiheitsgrade & Signifikanz (p-Wert) \\
Gesamtvergleich & 35,914 & 2 & $<0,001$ \\
$0-50 \%$ vs. $51-150 \%$ & 3,228 & 1 & 0,720 \\
$0-50 \%$ vs. $151-300 \%$ & 34,446 & 1 & $<0,001$ \\
$51-150 \%$ vs. $151-300 \%$ & 6,885 & 1 & $\mathbf{0 , 0 0 9}$ \\
\hline
\end{tabular}




\subsubsection{Einfluss der abgegebenen Schocks auf die Batterielaufzeit}

Von 2026 ICDs konnten Daten über die abgegebenen Schocks erhoben werden, 538 dieser Geräte wurden batteriebedingt ausgetauscht. Die Kaplan-Meier-Kurve zeigt die signifikant unterschiedlichen Batterielaufzeiten der Geräte, unterteilt nach der Anzahl abgegebener Schocks (Abbildung 8). Bei Geräten die zwei oder mehr Schocks abgegeben hatten, war eine Batterieerschöpfung signifikant eher erreicht worden als bei Geräten, die keine Schocks abgegeben hatten $(p=0,001)$. Außerdem war das Risiko für batteriebedingte Wechsel signifikant erhöht bei Geräten, die fünf oder mehr Schocks abgegeben hatten, gegenüber Geräten, die lediglich einen Schock abgegeben hatten $(p=0,038)$ (Tabelle 14).

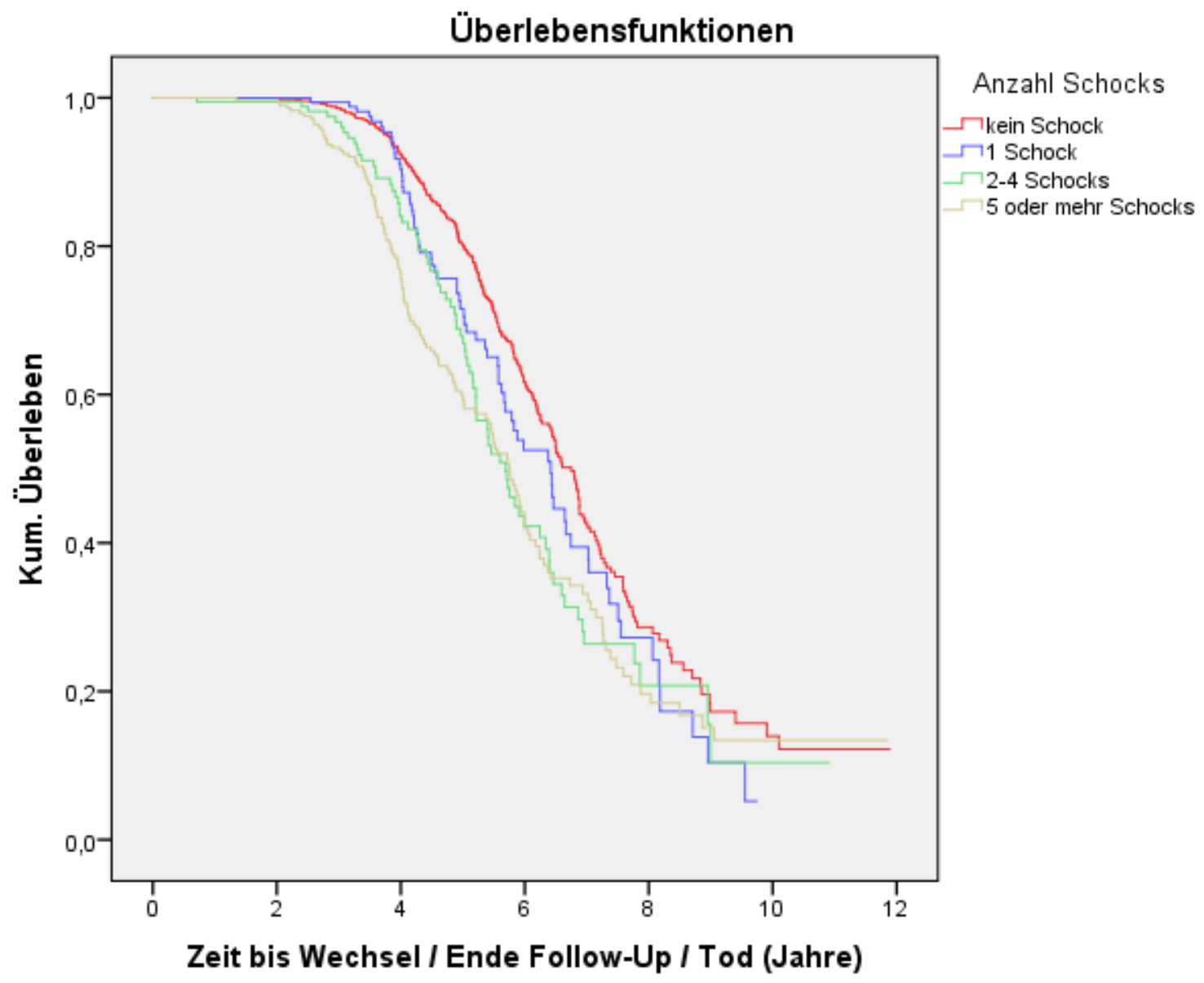

\begin{tabular}{|c|c|c|c|c|c|c|c|}
\hline 1208 & 935 & 524 & 174 & 36 & 8 & 0 & Kein Schock \\
\hline 239 & 185 & 119 & 38 & 9 & 0 & & 1 Schock \\
\hline 204 & 158 & 98 & 31 & 7 & 1 & 0 & $2-4$ Schocks \\
\hline 375 & 286 & 162 & 56 & 16 & 5 & 0 & $\geq 5$ Schocks \\
\hline
\end{tabular}

Abbildung 8: Einfluss der abgegebenen Schocks auf die Batterielaufzeit 
Tabelle 14: Einfluss der abgegebenen Schocks auf die Batterielaufzeit

\begin{tabular}{|c|c|c|c|}
\hline Log-Rank-Test & Chi-Quadrat & Freiheitsgrade & Signifikanz ( $p$-Wert) \\
\hline Gesamtvergleich & 29,633 & 3 & $<0,001$ \\
\hline Kein Schock vs. 1 Schock & 3,400 & 1 & 0,065 \\
\hline Kein Schock vs. 2-4 Schocks & 11,643 & 1 & 0,001 \\
\hline Kein Schock vs. $\geq 5$ Schocks & 25,363 & 1 & $<0,001$ \\
\hline 1 Schock vs. 2-4 Schocks & 1,464 & 1 & 0,226 \\
\hline 1 Schock vs. $\geq 5$ Schocks & 4,291 & 1 & 0,038 \\
\hline 2-4 Schocks vs. $\geq 5$ Schocks & 0,367 & 1 & 0,545 \\
\hline
\end{tabular}

\subsubsection{Einfluss der ICD-Generation auf die Batterielaufzeit}

Ein Vergleich der ICD-Generationen wurde für alle Gerätetypen durchgeführt. Insgesamt wurden 2408 ICDs betrachtet von denen 569 vor 2002 (n ERI = 295) und 1839 ab 2002 ( $\mathrm{E} \mathrm{ERI} \mathrm{=} \mathrm{292)} \mathrm{implantiert} \mathrm{wurden.} \mathrm{In} \mathrm{Tabelle} 15$ sind die funktionellen Eigenschaften der untersuchten ICDs aufgeteilt nach ICD-Generation aufgeführt. So waren beispielsweise 78,6 \% der ICDs, die in Göttingen vor 2002 implantiert wurden, VVI-Geräte (vs. 32,8 \% VVI ab 2002) und ein Großteil (67\%) waren Boston-ScientificGeräte (vs. 36 \% BSI ab 2002). Bei Geräten, die vor 2002 implantiert wurden, kam es signifikant eher zu einer Batterieerschöpfung als bei Geräten, die nach 2002 implantiert wurden (Abbildung 9, Tabelle 16). Noch deutlicher ist dieser Unterschied bei der separaten Betrachtung der VVI-Geräte. Hier wurden 1072 VVI-Geräte nach der Kaplan-Meier-Methode untersucht, von denen 447 vor 2002 ( $\mathrm{nERI}=224$ ) und $603 \mathrm{ab}$ 2002 (n ERI = 60) implantiert wurden (Abbildung 10). VVI-Geräte, die vor 2002 implantiert wurden, zeigten eine mediane Lebensdauer von 5,6 Jahren bis zur Batterieerschöpfung (95\% KI, 5,2-5,9 Jahre). VVI-Geräte, die nach 2002 implantiert wurden, zeigten eine mediane Batterielaufzeit von 8,3 Jahren (95\% KI, 8-8,6 Jahre). Bei DDD-Geräten und bei CRT-Ds zeigten sich keine signifikanten Batterielaufzeitunterschiede hinsichtlich der ICD-Generation (Tabelle 16). 
Tabelle 15: Eigenschaften der ICDs zum Einfluss der ICD-Generation auf die Batterielaufzeit

\begin{tabular}{|c|c|c|}
\hline Variable & $<2002(n=569)$ & $\geq 2002(n=1839)$ \\
\hline \multicolumn{3}{|l|}{ Hersteller } \\
\hline BSI & 381 (67 \%) & 662 (36 \%) \\
\hline MED & $187(32,9 \%)$ & 972 (52,9 \%) \\
\hline $\mathrm{BIO}$ & l & 203 (11\%) \\
\hline SJM & $1(0,2 \%)$ & $2(0,1 \%)$ \\
\hline \multicolumn{3}{|l|}{ Kammertyp } \\
\hline VVI & $447(78,6 \%)$ & $603(32,8 \%)$ \\
\hline DDD & 109 (19,2\%) & $542(29,5 \%)$ \\
\hline CRT-D & $13(2,3 \%)$ & $694(37,7 \%)$ \\
\hline \multicolumn{3}{|c|}{ Anzahl Schocks } \\
\hline Kein Schock & $160(28,1 \%)$ & 1048 (57 \%) \\
\hline 1 & $62(10,9 \%)$ & $177(9,6 \%)$ \\
\hline $2-4$ & $77(13,5 \%)$ & $127(6,9 \%)$ \\
\hline 5 oder mehr & $194(34,1 \%)$ & $181(9,8 \%)$ \\
\hline \multicolumn{3}{|c|}{ Stimulationsanteil } \\
\hline $0-50 \%$ & $235(41,3 \%)$ & 695 (37,8 \%) \\
\hline $51-150 \%$ & $59(10,4 \%)$ & 197 (10,7 \%) \\
\hline $151-300 \%$ & $75(13,2 \%)$ & $646(35,1 \%)$ \\
\hline
\end{tabular}




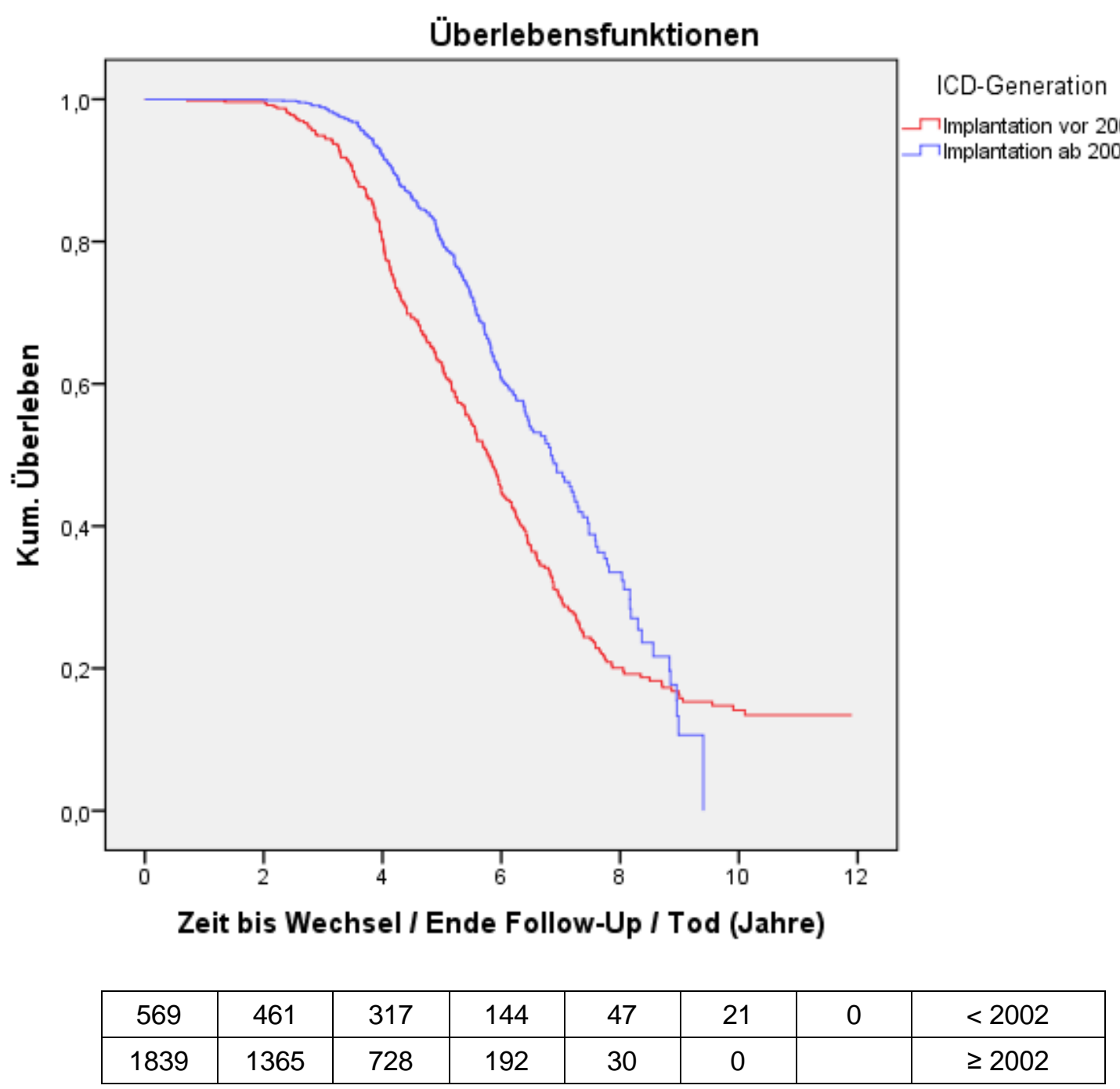

Abbildung 9: Einfluss der ICD-Generation auf die Batterielaufzeit

Tabelle 16: Einfluss der ICD-Generation auf die Batterielaufzeit

\begin{tabular}{llll}
\hline Log-Rank-Test & Chi-Quadrat & Freiheitsgrade & Signifikanz (p-Wert) \\
$<2002$ vs. $\geq 2002$ & 35,297 & 1 & $<0,001$ \\
VVI $<2002$ vs. $\geq 2002$ & 81,724 & 1 & $<0,001$ \\
DDD $<2002$ vs. $\geq 2002$ & 0,428 & 1 & 0,513 \\
CRT-D <2002 vs. $\geq 2002$ & 0,03 & 1 & 0,863 \\
\hline
\end{tabular}




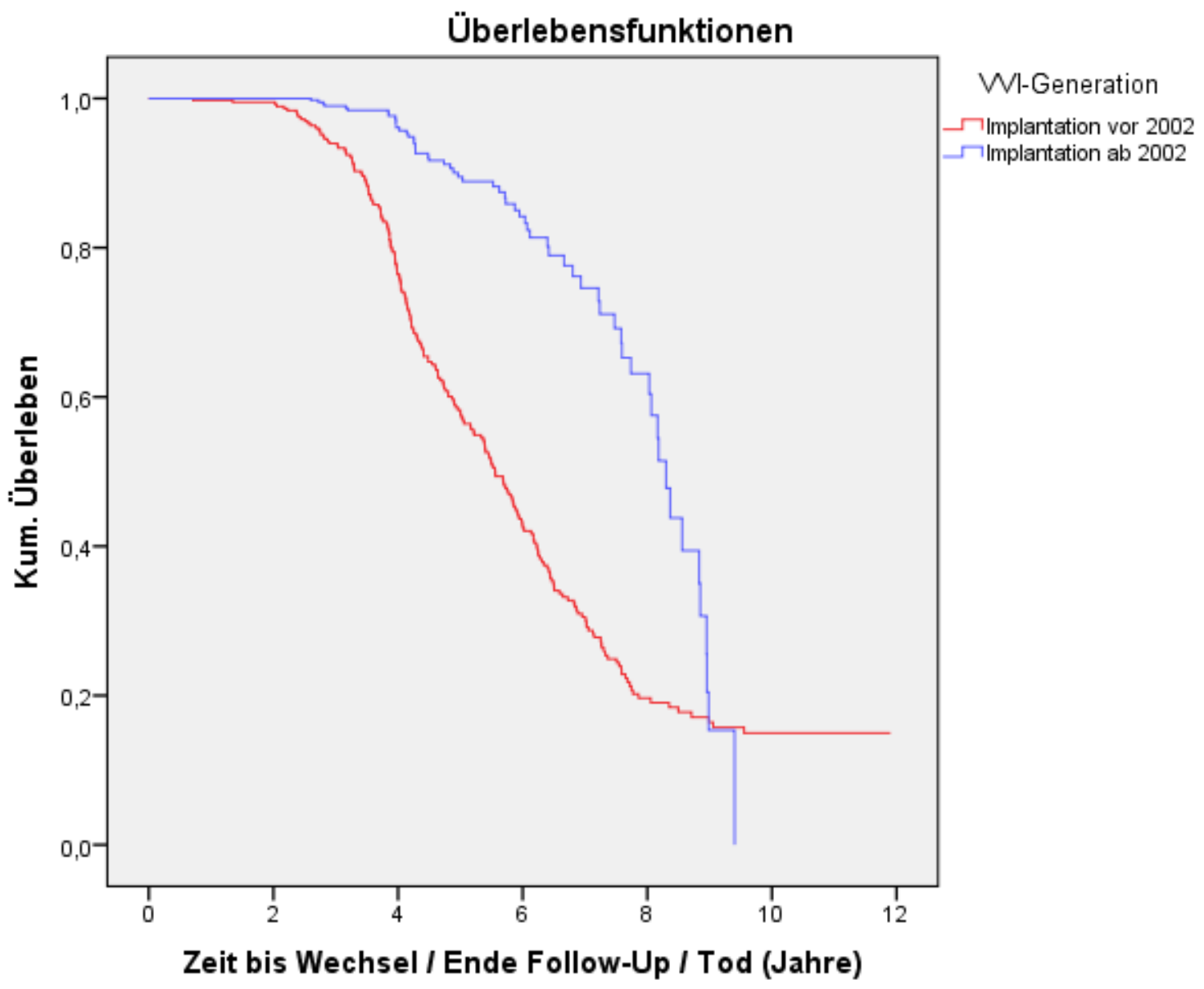

\begin{tabular}{|c|c|c|c|c|c|c|c|}
\hline 447 & 358 & 227 & 103 & 33 & 15 & 0 & $\mathrm{VVI}<2002$ \\
\hline 603 & 482 & 241 & 95 & 24 & 0 & & $\mathrm{VVI} \geq 2002$ \\
\hline
\end{tabular}

Abbildung 10: Einfluss der VVI-Generation auf die Batterielaufzeit 


\subsubsection{Univariate Cox-Regressionsanalyse zur Batterielaufzeit}

Tabelle 17: Univariate Cox-Regressionsanalyse zur Batterielaufzeit

\begin{tabular}{|c|c|c|c|}
\hline Variable & Hazard ratio & $95 \%$ Konfidenzintervall & P-Wert \\
\hline \multicolumn{4}{|l|}{ Gerätetyp } \\
\hline VVI & 0,832 & $0,705-0,983$ & 0,030 \\
\hline DDD & 0,879 & $0,730-1,057$ & 0,170 \\
\hline CRT-D & 1,537 & $1,269-1,861$ & $<0,001$ \\
\hline \multicolumn{4}{|l|}{ Hersteller } \\
\hline Boston Scientific & 1,556 & $1,323-1,830$ & $<0,001$ \\
\hline Medtronic & 0,640 & $0,544-0,753$ & $<0,001$ \\
\hline Biotronik & 1,036 & $0,607-1,770$ & 0,897 \\
\hline Alter über Median (62 Jahre) & 0,975 & $0,829-1,147$ & 0,759 \\
\hline Geschlecht männlich & 1,158 & $0,936-1,433$ & 0,177 \\
\hline Vorhofflimmern & 1,017 & $0,855-1,208$ & 0,853 \\
\hline KHK & 1,045 & $0,881-1,238$ & 0,614 \\
\hline DCM & 1,093 & $0,921-1,296$ & 0,309 \\
\hline Primärprophylaxe & 0,885 & $0,735-1,066$ & 0,198 \\
\hline Sekundärprophylaxe & 1,130 & $0,938-1,360$ & 0,198 \\
\hline Implantation vor 2002 & 1,650 & $1,396-1,949$ & $<0,001$ \\
\hline Implantation nach 2002 & 0,606 & $0,513-0,716$ & $<0,001$ \\
\hline \multicolumn{4}{|l|}{ Stimulationsanteil } \\
\hline $0-50 \%$ & 0,629 & $0,528-0,749$ & $<0,001$ \\
\hline $50-150 \%$ & 1,016 & $0,792-1,303$ & 0,902 \\
\hline $151-300 \%$ & 1,698 & $1,416-2,035$ & $<0,001$ \\
\hline \multicolumn{4}{|l|}{ Schocks } \\
\hline$\geq 1$ & 1,535 & $1,295-1,818$ & $<0,001$ \\
\hline$\geq 5$ & 1,508 & $1,244-1,828$ & $<0,001$ \\
\hline
\end{tabular}


Von den in Tabelle 17 gezeigten univariat analysierten Einflussfaktoren sind die verschiedenen ICD-Hersteller und ICD-Kammertypen mit einem unterschiedlich hohen Risiko für batteriebedingte Aggregatwechsel behaftet. Dies zeigt auch die KaplanMeier-Analyse. VVI-Geräte haben demnach ein geringeres Risiko für batteriebedingte Wechseloperationen $(H R=0,832)$. CRT-D-Geräte haben hierfür hingegen ein erhöhtes Risiko (HR = 1,537). Bei den Herstellern sind Medtronic-ICDs im Gegensatz zu Boston-Scientific-ICDs mit einem geringeren Risiko für batteriebedingte Aggregatwechsel verbunden ( $H R=0,640$ vs. $H R=1,556)$. Die Analyse der BiotronikGeräte ist auf Grund der geringeren Anzahl an batteriebedingten Wechseloperationen nicht signifikant. ICDs, die vor 2002 implantiert wurden, haben im Vergleich zu ICDs die ab 2002 implantiert wurden, ein signifikant erhöhtes Risiko für batteriebedingte Wechsel ( $H R=1,650$ vs. $H R=0,606)$. Die Generation der Geräte beinhaltet in dieser Analyse alle Kammertypen inklusive CRT-Ds. Eine separate Analyse der VVI-ICDs wurde wie oben beschrieben durchgeführt.

Ein geringer Stimulationsanteil (<50\%) ist signifikant mit einem geringen Risiko für Batterieerschöpfung verbunden $(\mathrm{HR}=0,629)$. Umgekehrt ist ein hoher Stimulationsanteil (151-300 \%) mit einem erhöhten Risiko für Batterieerschöpfung verbunden $(H R=1,698)$. Die Abgabe von Schocks geht in dieser Analyse signifikant $(p<0,001)$ mit einem erhöhten Risiko für Batterieerschöpfung einher. So liegt die Hazard Ratio bei Geräten, die einen oder mehr Schocks abgegeben haben, bei 1,535 (5 oder mehr Schocks: HR = 1,508). Folgende Faktoren haben keinen signifikanten Einfluss auf das Risiko eines batteriebedingten Aggregatwechsels: Ein hohes Lebensalter (Median $\geq 62$ Jahre), männliches Geschlecht, Vorhofflimmern, eine KHK oder DCM als kardiale Grunderkrankung sowie die ICD-Indikation bei Erstimplantation (Primärprophylaxe vs. Sekundärprophylaxe).

\subsubsection{Multivariate Cox-Regressionsanalyse zur Batterielaufzeit}

Alle Parameter, die in der univariaten Analyse einen $\mathrm{p}$-Wert $<0,1$ erreichten, wurden in die multivariate Analyse eingeschlossen. Die Analyse wurde mittels Rückwärtselimination durchgeführt. Tabelle 18 zeigt das hieraus resultierende finale Cox-Modell. Alle Parameter mit einem p-Wert < 0,05 wurden als signifikant erachtet. Zu sehen ist, dass CRT-D-Geräte ( $H R=1,818)$ sowie Geräte, die einen oder mehr Schocks abgegeben haben $(\mathrm{HR}=1,376)$, mit einem hohen Risiko für Batterieerschöpfung einhergehen. Des Weiteren sind Geräte die vor 2002 implantiert wurden, mit einem erhöhten Risiko $(H R=1,678)$ für batteriebedingte 
Wechseloperationen verbunden. Geräte mit einem niedrigen mittleren prozentualen Stimulationsanteil (0-50\%) ( $\mathrm{HR}=0,760)$, und Geräte der Firma Medtronic ( $\mathrm{HR}=$ $0,616)$ gehen mit einem signifikant erniedrigten Risiko für Batterieerschöpfung einher.

Tabelle 18: Finales Cox-Modell

\begin{tabular}{lcccc}
\hline Variable & Hazard ratio & $95 \%$ Konfidenzintervall & P-Wert & Wald \\
& & & & \\
CRT-D & 1,811 & $1,386-2,364$ & $<\mathbf{0 , 0 0 1}$ & 18,998 \\
Medtronic & 0,616 & $0,513-0,741$ & $<\mathbf{0 , 0 0 1}$ & 26,648 \\
$\geq$ 1 Schocks & 1,376 & $1,141-1,658$ & $\mathbf{0 , 0 0 1}$ & 11,164 \\
0-50\% Stimulation & 0,760 & $0,614-0,941$ & $\mathbf{0 , 0 1 2}$ & 6,362 \\
Implantation vor 2002 & 1,678 & $1,357-2,074$ & $<\mathbf{0 , 0 0 1}$ & 22,885 \\
\hline
\end{tabular}

\subsection{Gerätelaufzeitanalysen}

\subsubsection{Einfluss des ICD-Herstellers auf die Gerätelaufzeit}

Um die reale Laufzeit eines ICDs zu beurteilen wurden in dieser Analyse Wechseloperationen aus sämtlichen Gründen, nicht ausschließlich batteriebedingte Wechsel, berücksichtigt. 2405 ICDs wurden nach ihren Herstellern unterteilt und mit Hilfe der Kaplan-Meier-Methode untersucht (Abbildung 11). Auf Grund der geringen Anzahl wurden Geräte der Firma St. Jude Medical hier nicht untersucht.

Es zeigt sich ein signifikanter Unterschied der einzelnen Hersteller bezüglich der ICDLaufzeit (Tabelle 19). Ein Aggregatwechsel aus sämtlichen möglichen Gründen war bei Geräten der Fima Medtronic im Median nach 6,3 Jahren (95 \% KI, 6-6,6 Jahre) notwendig (vs. 6,9 Jahre mediane Batterielaufzeit). Geräte der Firma Boston Scientific / Guidant wurden im Median nach 5,5 Jahren (95 \% KI, 5,2-5,7 Jahre) ausgetauscht (vs. 5,8 Jahre mediane Batterielaufzeit) und bei ICDs der Firma Biotronik wurden Wechseloperationen nach 5,2 Jahren im Median (95\% KI, 5,1-5,4 Jahre) durchgeführt (vs. 5,2 Jahre mediane Batterielaufzeit). Im Vergleich liegt die reale Gerätelaufzeit der BSI- und MED-Geräte also unterhalb der Batterielaufzeit. Bei Biotronik-Geräten zeigte sich eine ähnliche mediane Geräte- und Batterielaufzeit. 


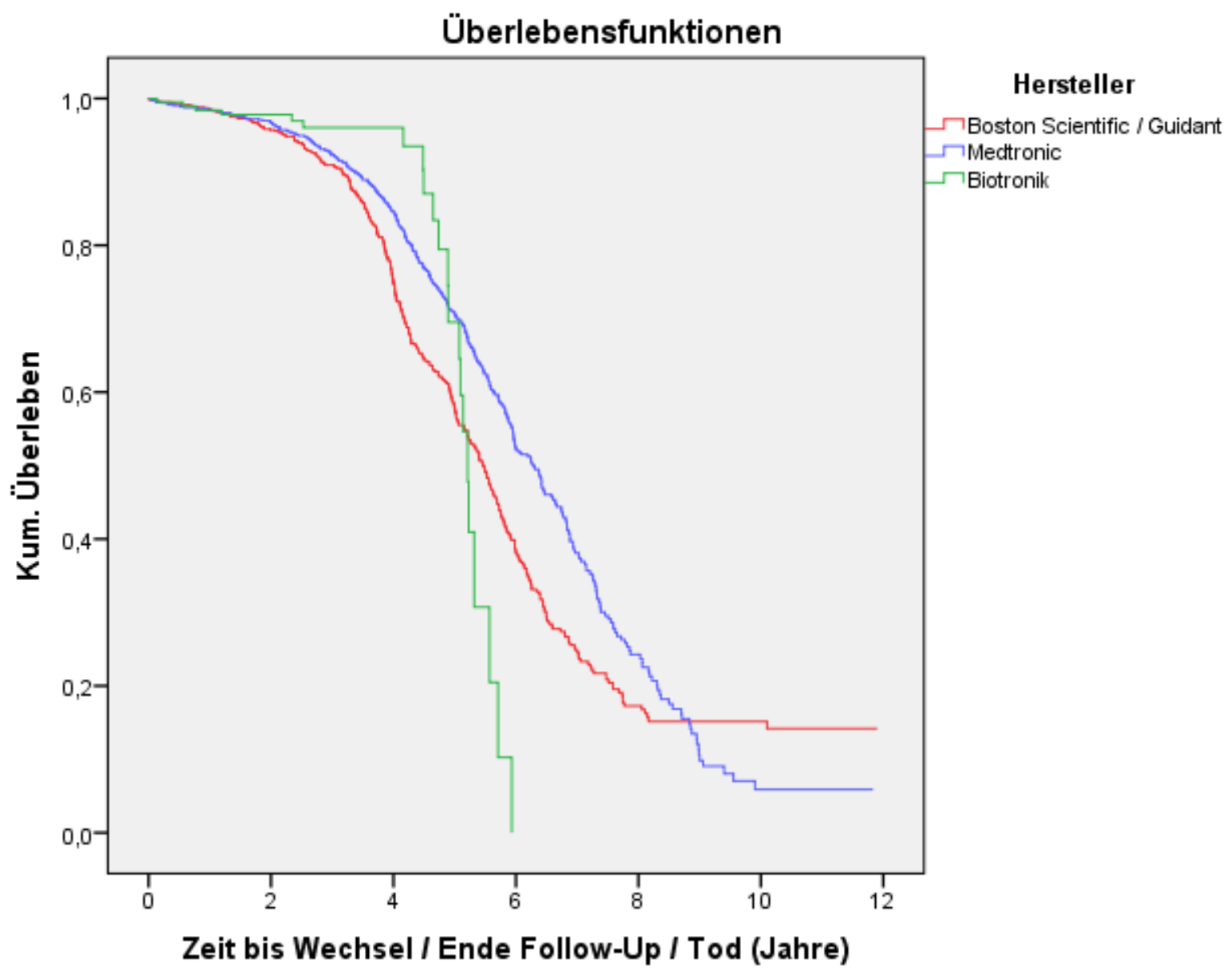

\begin{tabular}{|c|c|c|c|c|c|c|c|}
\hline 1043 & 781 & 397 & 127 & 33 & 16 & 0 & BSI \\
\hline 1159 & 914 & 605 & 209 & 44 & 5 & 0 & MED \\
\hline 203 & 130 & 43 & 0 & & & & BIO \\
\hline
\end{tabular}

Abbildung 11: Einfluss des Herstellers auf die Gerätelaufzeit

Tabelle 19: Einfluss des Herstellers auf die Gerätelaufzeit

\begin{tabular}{llll}
\hline Log-Rank-Test & Chi-Quadrat & Freiheitsgrade & $\begin{array}{l}\text { Signifikanz (p-Wert) } \\
<\mathbf{0 0 0 1}\end{array}$ \\
Gesamtvergleich & 18,616 & 2 & $<0,001$ \\
BSI vs. MED & 17,826 & 1 & 0,132 \\
BSI vs. BIO & 2,269 & 1 & 0,778 \\
MED vs. BIO & 0,079 & 1 & \\
\hline
\end{tabular}

\subsubsection{Einfluss des Kammertyps auf die Gerätelaufzeit}

Um den Einfluss des Kammertyps auf die reale Laufzeit der ICDs zu untersuchen wurden alle 2408 ICDs nach ihrem Kammertyp unterteilt und mit Hilfe der KaplanMeier-Methode analysiert (Abbildung 12). Hierbei zeigten sich signifikante Unterschiede im Gesamtvergleich sowie im Direktvergleich zwischen CRT-D-Geräten 
und VVI- und DDD-Geräten (Tabelle 20). Der Vergleich der Gerätelaufzeiten von VVIund DDD-Geräten ist wie in der Batterielaufzeitanalyse statistisch nicht signifikant. VVI-Geräte zeigten die längste Gerätelaufzeit von im Median 6,1 Jahren (95 \% KI, 5,86,4 Jahre) (vs. 6,8 Jahre mediane Batterielaufzeit). DDD-Geräte wurden nach im Median 5,9 Jahren (95 \% KI, 5,7-6,2 Jahre) (vs. 6,5 Jahre mediane Batterielaufzeit) und CRT-D-Geräte im Median nach 5,4 Jahren (95 \% KI, 5,2-5,6 Jahre) (vs. 5,6 Jahre mediane Batterielaufzeit) ersetzt. Es zeigt sich hier also auf alle Kammertypen bezogen eine kürzere reale Gerätelaufzeit im Vergleich zu den ermittelten medianen Batterielaufzeiten.

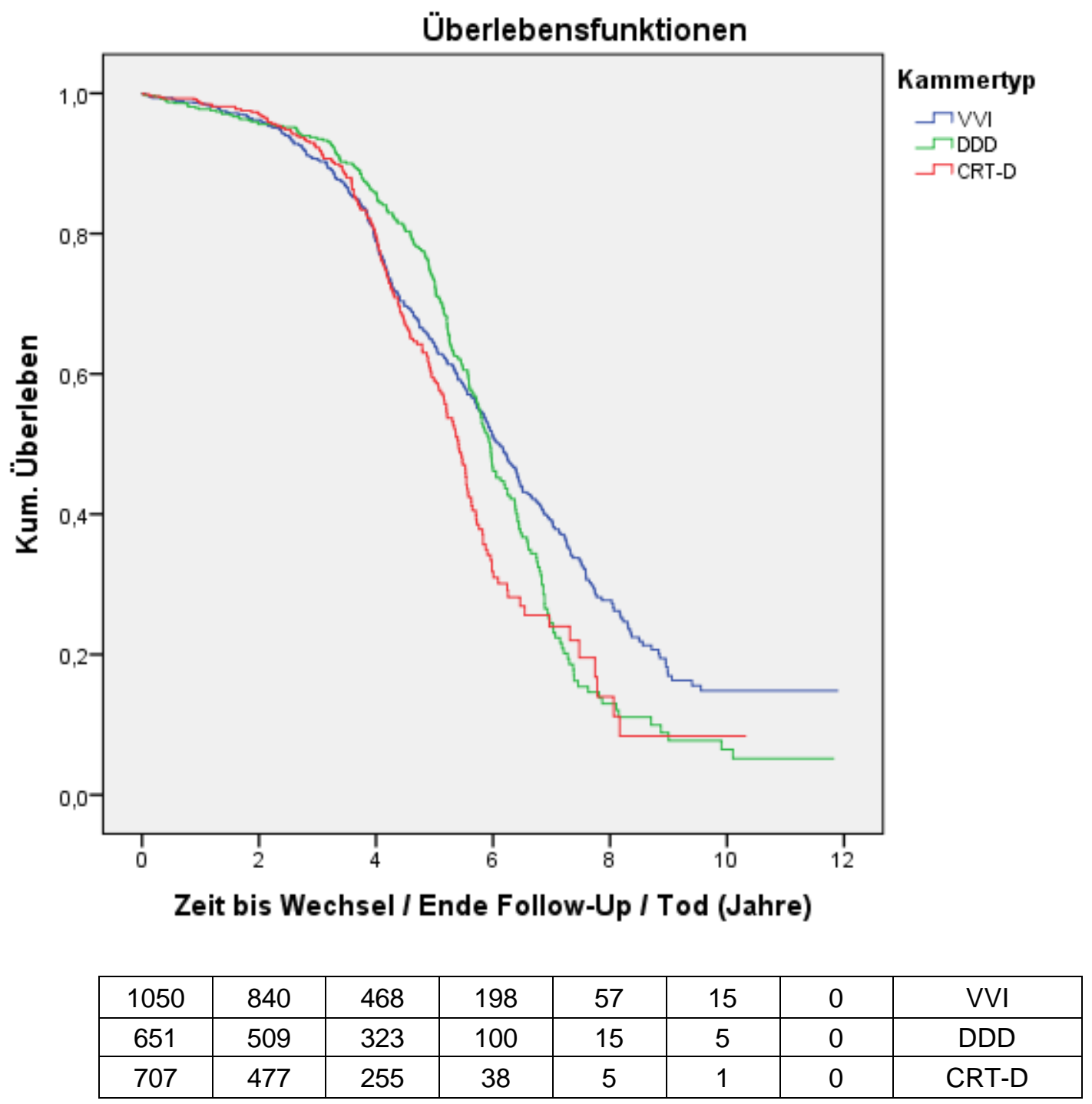

Abbildung 12: Einfluss des Kammertyps auf die Gerätelaufzeit 
Tabelle 20: Einfluss des Kammertyps auf die Gerätelaufzeit

\begin{tabular}{llll}
\hline Log-Rank-Test & Chi-Quadrat & Freiheitsgrade & Signifikanz (p-Wert) \\
Gesamtvergleich & 12,785 & 2 & $\mathbf{0 , 0 0 2}$ \\
VVI vs. DDD & 1,080 & 1 & 0,299 \\
VVI vs. CRT-D & 8,537 & 1 & $\mathbf{0 , 0 0 3}$ \\
DDD vs. CRT-D & 9,082 & 1 & $\mathbf{0 , 0 0 3}$ \\
\hline
\end{tabular}




\section{Diskussion}

\subsection{Ergebnisse}

Eines der Hauptergebnisse dieser retrospektiven monozentrischen Analyse sind die herstellerspezifischen Unterschiede in den ICD-Batterielaufzeiten. So zeigten VVI- und DDD-ICDs der Firma Medtronic eine längere Batterielaufzeit als diese Gerätetypen der Firma Boston Scientific / Guidant und der Firma Biotronik. Des Weiteren wurde gezeigt, dass CRT-D-Geräte eine kürzere Batterielaufzeit als VVI- und DDD-Geräte haben. Weitere Faktoren, die einen Einfluss auf die Batterielaufzeit von ICDs haben, sind die Anzahl abgegebener Schocks und der prozentuale Stimulationsanteil. ICDs, die vor 2002 implantiert wurden, zeigten kürzere Batterielaufzeiten als solche, die nach 2002 implantiert wurden. Besonders deutlich ist dieser Unterschied bei den VVI-Geräten.

\subsubsection{Einfluss der Hersteller}

Medtronic-ICDs zeigten in der Analyse aller Kammertypen gegenüber ICDs der Hersteller Boston Scientific / Guidant und der Firma Biotronik die längsten medianen Batterielaufzeiten ( 6,9 vs. 5,8 vs. 5,2 Jahre; $p<0,001$ ). Dies deckt sich größtenteils mit vorrausgegangenen Arbeiten, bei denen eine ähnliche ICD-Kohorte untersucht wurde. So ergab die Analyse von Horlbeck et al. (2012) längere Gerätelaufzeiten von Medtronic-ICDs (im Mittel 5,5 Jahre) gegenüber BSI-ICDs (4,6 Jahre) und BiotronikICDs (4,3 Jahre). Schaer et al. (2009) konnten ebenfalls längere mittlere Batterielaufzeiten von Medtronic-Geräten (7,6 Jahre) gegenüber BSI-Geräten (5 Jahre) und Geräten der Firma St. Jude Medical (3,8 Jahre) aufzeigen. Auch in der Arbeit von Thijssen et al. (2012) zeigten Medtronic-ICDs die längsten Batterielaufzeiten. Sie hielten im Mittel 5,8 Jahre, während BSI-Geräte eine durchschnittliche Batterielaufzeit von 5,3 Jahren und Biotronik-ICDs von 4,7 Jahren zeigten. In der Analyse von Biffi et al. (2008) konnten für VVI-Geräte des Herstellers Medtronic ebenfalls längere Batterielaufzeiten als für VVI-Geräte der Hersteller BSI und SJM dokumentiert werden. In einer aktuellen Analyse von Manolis et al. (2017), welche ebenfalls ICDs eines ähnlich breiten Implantationszeitraumes wie diese Arbeit untersuchten, ergaben sich für Medtronic-ICDs längere Batterielaufzeiten (5,9 Jahre) als für ICDs von BSI (4,9 Jahre) und Biotronik (4,3 Jahre). Bei Zanon et al. (2016) zeigten VVI- und DDD-ICDs des Herstellers SJM die längsten Batterielaufzeiten (6,7 Jahre), wobei für die anderen untersuchten Hersteller ähnliche Ergebnisse wie in 
dieser Arbeit gefunden werden konnten (MED 6,4 Jahre > BSI 6,3 Jahre > BIO 5,3 Jahre). Die Ursache für diese Überlegenheit von Medtronic-ICDs scheint laut Ansicht einiger Autoren in technischen Unterschieden begründet (Thijssen et al. 2012, Horlbeck et al. 2012, Schaer et al. 2009, Ellinor et al. 2003). Eine mögliche Ursache hierfür könnte beispielsweise die geringere Anzahl gesammelter intrakardialer Elektrogramme sein, die von Medtronic-ICDs vorgenommen wurden.

Nur die CRT-Ds betrachtet ergab sich in dieser Analyse kein signifikanter Unterschied hinsichtlich der beiden hauptsächlich untersuchten Hersteller Medtronic und BSI. Aktuelle Untersuchungen ergaben sogar längere CRT-D-Laufzeiten des Herstellers BSI im Vergleich zu Medtronic und anderen. In der Analyse von Alam et al. (2014) wurde eine Überlegenheit bezüglich der Batterielaufzeiten von CRT-Ds der Hersteller BSI und SJM gegenüber MED gezeigt. Hierbei ist allerdings zu bemerken, dass von den untersuchten CRT-Ds nur ca. $4 \%$ der BSI-Geräte batteriebedingt (ERI) ausgetauscht wurden. Bei den untersuchten Medtronic-CRT-Ds wurden dagegen $24 \%$ batteriebedingte Aggregatwechsel durchgeführt. Zum Vergleich lag dieses Verhältnis in der vorliegenden Analyse etwas ausgeglichener bei $17 \%$ BSI zu $23 \%$ bei MED. In einer Arbeit von Landolina et al. (2015) zeigten CRT-Ds des Herstellers BSI ebenfalls längere Laufzeiten als Medtronic-CRT-Ds. Dies könnte in den untersuchten ICD-Generationen begründet liegen. Bei Landolina et al. (2015) wurden so nur relativ neue ICDs untersucht, welche zwischen Januar 2008 und März 2010 implantiert wurden. In einer Arbeit von von Gunten et al. (2016) wird dies besonders deutlich. Ähnlich dieser Arbeit zeigte die Analyse der vor 2006 implantierten VVI- und DDD-ICDs längere Batterielaufzeiten von Medtronic-ICDs gegenüber ICDs der anderen untersuchten Hersteller (MED > SJM > BSI > Sorin > BIO > Intermedics). In der separat durchgeführten Analyse der nach 2006 implantierten ICDs zeigten nun ICDs aller Kammertypen des Herstellers BSI die längsten Batterielaufzeiten. Eine Ursache hierfür könnte in den von BSI ca. 2008 neu eingeführten LithiumMangandioxid-Batterien liegen, welche eine höhere Batteriekapazität besitzen als die Batterien anderer Hersteller (Boriani et al. 2016, Mond und Freitag 2014). Zu einem ähnlichen Ergebnis kamen auch Zanon et al. (2016), bei denen eine separate Analyse der CRT-D-Geräte ebenfalls die längsten Batterielaufzeiten für BSI-Geräte (5,8 Jahre) gegenüber MED-Geräten (4,5 Jahre) und BIO-Geräten (4,4 Jahre) ergab. Der Vorteil längerer Batterielaufzeiten von Medtronic-ICDs scheint sich also auf VVI- und DDDGeräte vorangegangener Generationen zu beschränken. 


\subsubsection{Einfluss des Kammertyps}

Der untersuchte Einfluss des Kammertyps auf die Batterielaufzeit von ICDs ergab, dass CRT-Ds kürzere Batterielaufzeiten als VVI- und DDD-Geräte haben (Median: CRT-D 5,6 Jahre; DDD 6,5 Jahre; VVI 6,8 Jahre). Dies zeigten auch die Ergebnisse einiger vorangegangener Laufzeitanalysen. So zeigten die Arbeiten von Biffi et al. (2008) und Schaer et al. (2009) ebenfalls kürzere Batterielaufzeiten für CRT-Ds gegenüber VVI- und DDD-ICDs. Bei Horlbeck et al. (2012) lagen die Gerätelaufzeiten von VVI-ICDs bei im Mittel 7 Jahren gegenüber einer Laufzeit von 5,7 Jahren bei DDDs und 4,4 Jahren bei CRT-Ds. Auch in der Arbeit von Thijssen et al. (2012) zeigten CRTDs mit 4,7 Jahren die kürzesten Batterielaufzeiten. Im Gegensatz zu dieser Analyse hatten bei Thijssen et al. (2012) DDD-Geräte mit 5,8 Jahren jedoch längere Batterielaufzeiten als VVI-Geräte (5,5 Jahre). In der Arbeit von von Gunten et al. (2016) waren nach 6 Jahren Follow-Up noch $76 \%$ der VVI-Geräte und $51 \%$ der DDDs gegenüber $43 \%$ der CRT-D-Geräte aktiv. Bei Zanon et al. (2016) ergab sich für VVIund DDD-ICDs eine mediane Laufzeit von 5,9 Jahren gegenüber einer medianen Laufzeit von 4,9 Jahren bei CRT-Ds. In einer Arbeit von Boriani et al. (2016), bei der lediglich BSI-Geräte analysiert worden sind, wurden für VVI-Geräte lange Laufzeiten von 13 Jahren, für DDDs von 12 Jahren und für CRT-Ds von 10 Jahren vorausgesagt. Auch bei Manolis et al. (2017) konnte für CRT-Ds eine kürzere Batterielaufzeit als für DDDs und VVIs dokumentiert werden (CRT-D 3,9 Jahre; DDD 4,9 Jahre; VVI 5,3 Jahre).

Die in allen Analysen aufgezeigte kürzere Batterielaufzeit bei den CRT-Ds kann auf den meist gewünscht hohen biventrikulären Stimulationsanteil zurückgeführt werden. Ein hoher Stimulationsanteil benötigt mehr Batterieenergie und ist somit signifikant mit einer geringeren Batterielaufzeit verbunden. Darüber hinaus ist die Reizschwelle der Sonde am linken Ventrikel gewöhnlich höher als im rechten Ventrikel, sodass eine erhöhte Stimulationsausgabe aufgebracht werden muss, was ebenfalls mit einer geringeren Batterielaufzeit bei CRT-Ds einhergehen könnte (Boriani et al. 2016).

\subsubsection{Gerätelaufzeitanalyse}

Die Gerätelaufzeit von ICDs, und damit die reale Lebenszeit, ist erwartungsgemäß deutlich kürzer als die errechnete Batterielaufzeit (Gesamtvergleich 5,8 vs. 6,4 Jahre). Das zeigt sich sowohl für die einzelnen Kammertypen (VVI 6,1 vs. 6,8 Jahre; DDD 5,9 vs. 6,5 Jahre; CRT-D 5,4 vs. 5,6 Jahre), als auch für die Hersteller Medtronic (6,3 vs. 
6,9 Jahre) und BSI (5,5 vs. 5,8 Jahre). Auch andere Arbeiten konnten diesen Unterschied aufzeigen, so lag beispielsweise in der Arbeit von Thijssen et al. (2012) die Gerätelaufzeit aller untersuchten ICDs bei im Mittel 5 Jahren gegenüber einer Batterielaufzeit von 5,5 Jahren. Die Ursache liegt darin begründet, dass bei der Gerätelaufzeitanalyse auch nicht batteriebedingte Aggregatwechsel berücksichtigt wurden. Batterieerschöpfung ist die häufigste $(82,7 \%)$ Ursache für Wechseloperationen. Bei 17,3\% der gewechselten ICDs lagen bereits vor einer drohenden Batterieerschöpfung andere Gründe für einen Wechsel vor. Dieser Anteil an nicht batteriebedingten Aggregatwechseln findet sich in etwa auch in anderen Arbeiten (Knops et al. 2009: 24,1\%; Thijssen et al. 2012: $28 \%$ nicht batteriebedingte Aggregatwechsel). Hieraus ergibt sich, dass es weiterhin größtmöglicher Anstrengungen bedarf, die Ursachen für nicht batteriebedingte Aggregatwechsel, wie beispielsweise Infektionen, so gut wie möglich zu verhindern. Denn für Patienten und Gesundheitssystem ist letztlich die reale Gerätelaufzeit entscheidend, und nicht die theoretische Batterielaufzeit eines ICDs.

\subsubsection{Einfluss des Stimulationsanteils}

Ein ICD mit einem mittleren prozentualen Stimulationsanteil von unter $50 \%$ hat ein geringeres Risiko für batteriebedingte Aggregatwechsel als Geräte mit einem hohen ventrikulären Stimulationsanteil. Diesen Zusammenhang zeigten auch andere Arbeiten. So war bei Thijssen et al. (2012) ein 10\%-iger Anstieg des Stimulationsanteils mit einer statistisch signifikant kürzeren Batterielaufzeit verbunden. Auch Schaer et al. (2009) und Horlbeck et al. (2012) konnten diesen Zusammenhang aufzeigen. So lag bei Horlbeck et al. (2012) die mittlere ICD-Batterielaufzeit von VVIund DDD-Geräten mit einem mittleren rechtsventrikulären Stimulationsanteil von unter $50 \%$ bei 5,5 Jahren gegenüber einer Batterielaufzeit von 4,7 Jahren bei VVI- und DDD-Geräten mit einem Stimulationsanteil von $>50 \%$. Auch hier war eine $10 \%$-ige Abnahme des Stimulationsanteils mit einer signifikant verlängerten Batterielaufzeit verbunden. In der Arbeit von Zanon et al. (2016) konnte ein hoher Stimulationsanteil als signifikanter, unabhängiger Vorhersagewert für kürzere Batterielaufzeiten gefunden werden. Bei Boriani et al. (2016) war ein hoher Stimulationsanteil bei DDDund CRT-D-Geräten mit schnellerer Batterieentladung verbunden. Demgegenüber konnten Landolina et al. (2015) keinen Einfluss des Stimulationsanteils auf die Batterielaufzeiten von CRT-Ds nachweisen. 
Ein hoher Stimulationsanteil hat darüber hinaus noch weitere Nachteile für die Patienten. Einige Arbeiten konnten zeigen, dass ein hoher mittlerer Stimulationsanteil zu einer erhöhten Mortalität führen kann. Dadurch kann sogar der mit dem ICD verbundene Überlebensvorteil aufgehoben werden (Barsheshet et al. 2011; Benditt et al. 2011, Wilkoff et al. 2002). Folglich sollte der Stimulationsanteil, sofern möglich, so gering wie möglich gehalten werden.

\subsubsection{Einfluss der abgegebenen Schocks}

Geräte, die keine Schocks abgegeben haben, haben ein niedrigeres Risiko für batteriebedingte Wechseloperationen als Geräte, die fünf oder mehr Schocks abgegeben haben. So zeigten Geräte aller Kammertypen, die fünf oder mehr Schocks abgegeben haben, mediane Batterielaufzeiten von 5,7 Jahren gegenüber einer medianen Batterielaufzeit von 6,7 Jahren bei Geräten, die keine Schocks abgegeben haben $(p<0,001)$. Die Ursache für diesen Zusammenhang liegt vermutlich darin, dass eine an den Schock gekoppelte Kondensatoraufladung die Batterie belastet. In einer Arbeit von Boriani et al. (2016) konnte in einem prospektiven Modell gezeigt werden, dass Schocks bei allen untersuchten Kammertypen mit einer schnelleren Batterieentladung verbunden sind.

In anderen Arbeiten wurde der Einfluss von Schocks auf die Batterielaufzeit zwar vermutet, konnte aber nicht signifikant gezeigt werden. So haben in der Arbeit von Schaer et al. (2009) Schocks keinen statistisch signifikanten Einfluss auf die Batterielaufzeit, wobei hier Schocks nach ineffektiver antitachykarder Stimulation nicht registriert wurden. Bei Thijssen et al. (2012), Biffi et al. (2008) und Landolina et al. (2015) hatte die Anzahl abgegebener Schocks keinen signifikanten Einfluss auf die ICD-Batterielaufzeit. Bei Landolina et al. (2015) wurde hierbei jedoch in der univariaten Analyse lediglich verglichen, ob Schocks abgegeben wurden oder nicht; ob multiple Schocks einen Einfluss auf die Batterielaufzeit haben wurde nicht separat untersucht. Der in dieser Arbeit gezeigte Einfluss von Schocks auf die Batterielaufzeit bedarf also weiterer Untersuchungen.

\subsubsection{Einfluss der ICD-Generation}

In der multivariaten Cox-Regressionsanalyse aller ICD-Typen, konnte ein Einfluss der ICD-Generation auf die Batterielaufzeit festgestellt werden. Neuere ICDs (Implantation ab 2002) haben im Gegensatz zu älteren ICDs (Implantation vor 2002) längere 
Batterielaufzeiten (6,8 vs. 5,8 Jahre). Dieses Ergebnis zeigte auch die Arbeit von Thijssen et al. (2012). Hier wurden bezüglich des Einflusses der ICD-Generation auf die Batterielaufzeit ebenfalls alle drei Kammertypen berücksichtigt. ICDs, die vor 2002 implantiert wurden, zeigten eine mittlere Batterielaufzeit von 4,9 Jahren gegenüber 5,6 Jahren bei Geräten, die ab 2002 implantiert wurden. Manolis et al. (2017) konnten ebenfalls eine verbesserte mittlere Batterielaufzeit von neueren ICDs (5 Jahre) gegenüber älteren ICDs (4,6 Jahre) dokumentieren. Bei Landolina et al. (2015) konnten für CRT-Ds aller Hersteller, die ab 2007 implantiert wurden, bessere Batterielaufzeiten dokumentiert werden als für CRT-Ds, die vor 2007 implantiert wurden. Horlbeck et al. (2012) stellten nur eine geringfügige Verbesserung der Batterielaufzeiten neuerer VVI- und DDD-Geräte (Implantation ab 2002) im Vergleich zu älteren Geräten (Implantation vor 2002) fest (<2002: 5,2 Jahre vs. $\geq 2002: 5,5$ Jahre). Schaer et al. (2009) konnten hingegen keinen Einfluss der ICD-Generation auf die Batterielaufzeit der ICDs feststellen. Dies erklärten sie durch die hohe Anzahl an CRT-Ds in der untersuchten Gruppe der neueren ( $\geq 2002$ ) ICDs, welche eine kürzere Batterielaufzeit aufwiesen. Um dieses Artefakt auszublenden wurde in dieser Untersuchung die Generation der VVI-Geräte zusätzlich separat untersucht. Die Analyse für alle Gerätetypen zeigte eine signifikant längere Batterielaufzeit bei neuen Geräten im Gegensatz zu Alten. Dies trifft ebenfalls für die Analyse der VVI-Subgruppe zu. Hier ist der Unterschied zwischen alten und neuen Geräten allerdings noch deutlicher zu erkennen (5,6 Jahre vs. 8,3 Jahre mediane Batterielaufzeit). Neuere CRT-Ds haben verbesserte technische Möglichkeiten, aber kurze Batterielaufzeiten. Dies führt dazu, dass die Verbesserung der Batterielaufzeiten neuerer ICDGenerationen insgesamt betrachtet nicht so deutlich ist, wie sie z. B. anhand der VVIGeräte gezeigt werden konnte. Ein Vorteil für die Patienten, nämlich Risikosenkung durch weniger operative Eingriffe auf Grund von Wechseloperationen, wird durch neue ICD-Generationen folglich nur teilweise erreicht. Darüber hinaus konnten von Gunten et al. (2016) zeigen, dass sich die Batterielaufzeit neuerer ICD-Generationen im Vergleich mit älteren Geräten zwar verbessert hat, die Lebenserwartung der Patienten aber insgesamt noch mehr gestiegen ist als die Batterielaufzeit der ICDs, sodass mehr Wechseloperationen notwendig werden. So lag das 5-Jahres-Überleben bei Patienten mit einem VVI-ICD 5 \% über der ICD-Batterielaufzeit. Bei DDD-Geräten betrug dieser Unterschied $15 \%$ und bei CRT-Ds $23 \%$. 


\subsection{Methode}

\subsubsection{Schwächen der Arbeit}

Da es sich bei dieser Arbeit um eine retrospektive Analyse von prospektiv erhobenen Daten handelt, war nicht zu vermeiden, dass einige Daten nicht ganz vollständig zu erheben waren. Diese mussten von der Analyse ausgeschlossen werden. Da in dieser Analyse die Batterielaufzeit von ICDs untersucht wurde, war es notwendig ICDs in die Analyse einzubeziehen, bei denen bereits batteriebedingte oder nicht batteriebedingte Aggregatwechsel stattgefunden hatten. Es kann somit keine Aussage zur neuesten ICD-Generation gemacht werden, da hier noch keine Wechseloperationen stattgefunden haben. So wurden beispielsweise keine subkutanen ICDs analysiert, da diese Modelle erst zum Ende der Datenerhebung entwickelt und implantiert wurden (Dabiri Abkenari et al. 2011).

An der Universität Göttingen wurden überwiegend ICDs der Firmen Medtronic und Boston Scientific / Guidant implantiert. Biotronik-ICDs wurden erst ab dem Jahr 2006 implantiert. Hieraus resultiert eine geringe Zahl an Aggregatswechseln für Geräte dieser Firma zum Zeitpunkt der Datenaufnahme. Auf Grund der geringen Stichprobe für diesen Hersteller sind die hierfür erhobenen Daten nur begrenzt signifikant. Geräte der Firma St. Jude Medical wurden nicht analysiert, da lediglich Informationen zu drei implantierten Geräten vorlagen. Auch andere Hersteller wie „ELA Medical Inc. / Sorin Group" konnten nicht in die Datenanalyse eingeschlossen werden, da ihre Geräte an der Universitätsklinik Göttingen nicht implantiert wurden. In dieser Analyse wurde der prozentuale Stimulationsanteil jedes Gerätes untersucht, der am letzten Follow-UpDatum gespeichert wurde. Dies kann lediglich als Annäherung an die zuvor erfolgte Stimulation ausgelegt werden. Außerdem wurden unterschiedlich viele ICD-Typen eines jeden Hersteller implantiert. Beispielsweise wurden mehr VVI-Geräte der Firma BSI als VVI-Geräte der Firma Medtronic in die Analyse eingeschlossen. Retrospektiv war es nicht möglich, die Anzahl abgegebener Schocks, die ein Patient während des gesamten Follow-Ups erhalten hat, den einzelnen ICDs die dieser Patient hatte, zuzuordnen. Deshalb wurde die Anzahl abgegebener Schocks, die ein Patient während des gesamten Follow-Ups erhalten hat, addiert und auf die Gesamtzahl aller ICDs die ein Patient hatte, verteilt. Darüber hinaus könnten weitere Parameter wie beispielsweise die Stimulationsausgabe einen Einfluss auf die ICD-Batterielaufzeit haben, was in dieser Arbeit nicht untersucht wurde. 


\subsubsection{Stärken der Arbeit}

Eine Stärke der Arbeit ist die mit 2408 relativ hohe Anzahl an untersuchten ICDs. Verglichen mit anderen retrospektiven Analysen haben lediglich die Arbeit von Thijssen et al. (2012) mit 4673 untersuchten ICDs und die Arbeit von von Gunten et al. (2016) eine größere Stichprobe $(n=4881)$. Bei Landolina et al. (2015) waren es 1726 untersuchte ICDs, bei Horlbeck et al. (2012) waren es 1502 ICDs und bei Schaer et al. (2009) 644. Eine weitere Stärke ist die vergleichsweise gute Datenqualität: Es handelt sich um eine Single-Center-Studie und die prospektiven Follow-Up-Abfragen und ICDProgrammierungen wurden über die gesamte Follow-Up-Dauer von derselben Person durchgeführt. Somit gibt es keine personenbezogenen Differenzen bezüglich der Dokumentation oder der ICD-Programmierung, was eine gute Vergleichbarkeit zur Folge hat.

\subsection{Schlussfolgerung}

Die Batterielaufzeit von ICDs hat einen hohen Stellenwert für Patienten. Eine Befragung von 156 ICD-Patienten von Wild et al. (2004) ergab, dass fast alle Patienten (90 \%) sich eher einen länger haltenden und dafür größeren ICD wünschten, als ein kleineres Gerät, das eher ausgetauscht werden muss. Darüber hinaus konnten verschiedene Arbeiten zeigen, dass lebensrettende Schocks auch noch nach vielen Jahren ohne appropriate Therapie auftreten können. So profitieren auch die Patienten von einem ICD-Aggregatwechsel, deren erster ICD zuvor keine appropriate Therapie abgegeben hatte (Van Welsenes et al. 2011b, Yap et al. 2014).

Um ein besseres Kosten-Nutzen Verhältnis zu erreichen, ist es von Bedeutung, die Batterielaufzeiten von ICDs zu verbessern. Hauser (2005) beschrieb, dass es zukünftig zu einer stetig größer werdenden Differenz zwischen ICD-Laufzeit und dem Patientenüberleben kommen wird. Dies führt wiederrum zu vermehrten Kosten. So zeigte er, dass eine hypothetische Gerätelaufzeit von zehn Jahren einen Großteil der ICD-Aggregatwechsel einsparen würde. Dies liegt daran, dass nur ca. 40 \% der ICDPatienten mit linksventrikulärer Dysfunktion die folgenden zehn Jahre überleben würden. Boriani et al. (2013) konnten in einer Modellstudie zeigen, dass mit einer theoretischen Gerätelaufzeit von 15 Jahren 29-34 \% der täglichen Kosten, je nach klinischem Szenario, gespart werden könnten. Ramachandra (2010) analysierte Krankendaten von 164 US-Veteranen, denen im Zeitraum von 1992 bis 2007 ein ICD implantiert wurde. Es konnte gezeigt werden, dass bei einer theoretischen ICD- 
Batterielaufzeit von sieben bzw. neun Jahren, ca. $58 \%$ bzw. $84 \%$ der Aggregatwechsel hätten vermieden werden können. Hierbei wären theoretische Kosteneinsparungen von bis zu 980.500 US-Dollar möglich gewesen.

Aus der Literatur ist bekannt, dass es einen signifikanten Unterschied zwischen der realen Batterielaufzeit von ICDs und den von den jeweiligen Herstellern prognostizierten Batterielaufzeiten gibt (Schaer et al. 2009; Shafat et al. 2013). In einer Arbeit von Shafat et al. (2013) erreichte mehr als ein Viertel der untersuchten ICDs nicht $80 \%$ der für sie vom Hersteller prognostizierten Batterielaufzeit.

Die Ergebnisse dieser Arbeit können einen Teil dazu beitragen, die Einflussfaktoren auf ICD-Batterielaufzeiten zu verstehen. Um zukünftig noch genauere Aussagen zu den Einflussfaktoren auf ICD-Batterielaufzeiten treffen zu können, bedarf es aber weiterer Analysen und größerer Datenbanken. In jedem Fall hat diese Arbeit mit denen aus der Literatur bekannten Arbeiten gemeinsam, dass die ermittelten Batterielaufzeiten nicht als befriedigend wahrgenommen werden können. Es sollte versucht werden, die entdeckten Einflussfaktoren, wie die Anzahl abgegebener Schocks und den Stimulationsanteil so gering wie möglich zu halten. Dies richtet sich einerseits an den klinisch tätigen Arzt, der die ICDs entsprechend programmieren sollte. Ein Weg dies erreichen zu können ist die in der MADIT-RIT-Studie (Multicenter Automatic Defibrillator Implantation Trial - Reduce Inappropriate Therapy) veröffentlichte Möglichkeit durch eine neuere Form der ICD-Programmierung mittels verzögerter Therapie die Inzidenz von inappropriaten Schocks zu reduzieren (Moss et al. 2012). Es richtet sich andererseits aber auch an die Industrie, die weitere Bemühungen vornehmen sollte die Batterielaufzeiten von ICDs zu verbessern.

Darüber hinaus konnten herstellerspezifische Unterschiede in der Batterielaufzeit von ICDs gezeigt werden. Diese sollten bei der Auswahl der zu implantierenden Geräte im Sinne der Patienten berücksichtigt werden. Außerdem wäre eine bessere Vergütung lang haltender ICDs zu diskutieren, um letztlich Folgekosten sparen zu können und einen industriellen Anreiz für längere Batterielaufzeiten zu schaffen. Ein weiterer beispielhafter Ansatz, die Batterielaufzeit zu verbessern, ist der vermehrte Einsatz von Home Monitoring. Hier müssen die Patienten seltener zu Routinekontrollen kommen, da die ICDs per Fernübertragung abgefragt werden können. Guedon-Moreau et al. (2013) konnten zeigen, dass der Einsatz von Home Monitoring zu weniger appropriaten und inappropriaten Schocks führt. Dies ist verbundenen mit einer geringeren Anzahl an Kondensatoraufladungen der ICDs, wodurch verlängerte Batterielaufzeiten erreicht werden können. In einer aktuellen Arbeit von Varma et al. 
(2018) konnte dies ebenfalls aufgezeigt werden. Von den 1339 untersuchten VVI- und DDD-ICDs des Herstellers Biotronik zeigte sich hier eine um $12 \%$ relativ verbesserte Batterieleistung nach 15 Monaten in der Gruppe der mittels Home Monitoring $(n=908)$ nachgesorgten Patienten gegenüber den konventionell nachgesorgten Patienten ( $\mathrm{n}=$ 431). Ein interessanter technischer Ansatz zur Verbesserung der ICD-Batterielaufzeit / Minimierung von Aggregatwechseln wäre die Entwicklung eines transkutan wieder aufladbaren ICDs. Im Tierversuch konnten in der Vergangenheit hiermit erste Ergebnisse erzielt werden (Detsch et al. 2006). 


\section{Zusammenfassung}

Durch die erweiterten Indikationen für implantierbare Kardioverter-Defibrillatoren (ICD) bei der Prophylaxe des plötzlichen Herztodes werden immer mehr ICDs implantiert. Die dabei oft notwendigen Aggregatwechsel gehen mit erheblichen Risiken für die Patienten und Kosten für das Gesundheitssystem einher. Ein Großteil der Wechseloperationen erfolgt auf Grund von Batterieversagen. Die vorliegende Arbeit untersuchte deshalb potenzielle Einflussfaktoren auf Batterielaufzeiten von ICDs. Es handelte sich um eine monozentrische, retrospektive, statistische Analyse aller Patienten, die zwischen März 1987 und Oktober 2010 einen ICD an der Universitätsklinik Göttingen implantiert bekommen haben. Es wurden Daten zu 2408 ICDs erhoben. Mit Hilfe der Kaplan-Meier-Methode und Cox-Regressionsanalysen wurden die Batterielaufzeiten der ICDs und ihre Einflussfaktoren untersucht. Hierbei ergaben sich deutliche Batterielaufzeitunterschiede bezüglich der ICD-Hersteller und Kammertypen. Ein- und Zweikammer-Geräte der Firma Medtronic zeigten längere Batterielaufzeiten als Geräte der Firma Boston Scientific und der Firma Biotronik. ICDs mit einer hohen Stimulationsrate und vielen abgegebenen Schocks, sowie Geräte zur kardialen Resynchronisationstherapie plus Defibrillator (CRT-D) waren signifikant mit einem höheren Risiko für Batterieerschöpfung assoziiert. Neuere ICD-Generationen zeigten längere Batterielaufzeiten als ältere Generationen. Außerdem war die reale Laufzeit der Geräte erwartungsgemäß kürzer als die Batterielaufzeit.

Zusammenfassend erscheinen die Batterielaufzeiten von ICDs noch nicht ausreichend. Die Batterielaufzeitunterschiede zwischen den verschiedenen Herstellern sollten bei der Implantation berücksichtigt werden. Außerdem sollten die mit einer kürzeren Batterielaufzeit einhergehenden Einflussfaktoren, wie beispielsweise die Anzahl abgegebener Schocks und der mittlere Stimulationsanteil so gering wie möglich gehalten werden. Weitere klinische Forschung zur Evaluation von Einflussfaktoren auf die Batterielaufzeit von ICDs erscheint ebenso notwendig wie die Entwicklung länger haltender Batterien. 


\section{Literaturverzeichnis}

Abraham WT, Fisher WG, Smith AL, Delurgio DB, Leon AR, Loh E, Kocovic DZ, Packer M, Clavell AL, Hayes DL et al. (2002): Cardiac resynchronization in chronic heart failure. N Engl J Med $\underline{346}, 1845-$ 1853

Alam MB, Munir MB, Rattan R, Flanigan S, Adelstein E, Jain S, Saba S (2014): Battery longevity in cardiac resynchronization therapy implantable cardioverter defibrillators. Europace $\underline{16}, 246-251$

AVID (Antiarrhythmics versus Implantable Defibrillators) Investigators (1997): A comparison of antiarrhythmic-drug therapy with implantable defibrillators in patients resuscitated from near-fatal ventricular arrhythmias. N Engl J Med $\underline{337}, 1576-1584$

Bardy GH, Ivey TD, Allen MD, Johnson G, Mehra R, Greene HL (1989): A prospective randomized evaluation of biphasic versus monophasic waveform pulses on defibrillation efficacy in humans. J Am Coll Cardiol 14, 728-733

Bardy GH, Johnson G, Poole JE, Dolack GL, Kudenchuk PJ, Kelso D, Mitchell R, Mehra R, Hofer B (1993): A simplified, single-lead unipolar transvenous cardioversion- defibrillation system. Circulation $\underline{88}, 543-547$

Bardy GH, Lee KL, Mark DB, Poole JE, Packer DL, Boineau R, Domanski M, Troutman C, Anderson J, Johnson $G$ et al. (2005): Amiodarone or an implantable cardioverter-defibrillator for congestive heart failure. N Engl J Med 352, 225-237

Barsheshet A, Moss AJ, McNitt S, Jons C, Glikson M, Klein HU, Huang DT, Steinberg JS, Brown MW, Zareba $W$ et al. (2011): Long-term implications of cumulative right ventricular pacing among patients with an implantable cardioverter-defibrillator. Heart Rhythm $\underline{8}, 212-218$

Begley DA, Mohiddin SA, Tripodi D, Winkler JB, Fananapazir L (2003): Efficacy of implantable cardioverter defibrillator therapy for primary and secondary prevention of sudden cardiac death in hypertrophic cardiomyopathy. Pacing Clin Electrophysiol 26, 1887-1896

Benditt DG, Sakaguchi S, Jhanjee R (2011): Right ventricular pacing in MADIT-II: a risk factor for increased mortality, or mainly a marker of increased risk. Heart Rhythm $\underline{8}, 219-220$ 
Biffi M, Ziacchi M, Bertini M, Sangiorgi D, Corsini D, Martignani C, Diemberger I, Boriani G (2008): Longevity of implantable cardioverter-defibrillators: implications for clinical practice and health care systems. Europace 10, 1288-1295

Boriani G, Braunschweig F, Deharo JC, Leyva F, Lubinski A, Lazzaro C (2013): Impact of extending device longevity on the long-term costs of implantable cardioverter-defibrillator therapy: a modelling study with a 15-year time horizon. Europace $\underline{15}, 1453-1462$

Boriani G, Ritter P, Biffi M, Ziacchi M, Diemberger I, Martignani C, Valzania C, Valsecchi S, Padeletti L, Gadler F (2016): Battery drain in daily practice and medium-term projections on longevity of cardioverterdefibrillators: an analysis from a remote monitoring database. Europace 18, 1366-1373

Borleffs CJ, Thijssen J, De Bie MK, Van Rees JB, Van Welsenes GH, Van Erven L, Bax JJ, Cannegieter SC, Schalij MJ (2010): Recurrent implantable cardioverter-defibrillator replacement is associated with an increasing risk of pocket-related complications. Pacing Clin Electrophysiol $\underline{33}$, 1013-1019

Bristow MR, Saxon LA, Boehmer J, Krueger S, Kass DA, De Marco T, Carson P, DiCarlo L, DeMets D, White BG et al. (2004): Cardiac-resynchronization therapy with or without an implantable defibrillator in advanced chronic heart failure. N Engl J Med 350, 2140-2150

Buxton AE, Lee KL, Fisher JD, Josephson ME, Prystowsky EN, Hafley G (1999): A randomized study of the prevention of sudden death in patients with coronary artery disease. N Engl J Med $\underline{341}, 1882-$ 1890

Cannom DS, Prystowsky EN (2004): Evolution of the implantable cardioverter defibrillator. J Cardiovasc Electrophysiol 15, 375-385

Connolly SJ, Gent M, Roberts RS, Dorian P, Roy D, Sheldon RS, Mitchell LB, Green MS, Klein GJ, O Brien B (2000): Canadian implantable defibrillator study (CIDS): a randomized trial of the implantable cardioverter defibrillator against amiodarone. Circulation 101, 1297-1302

Connolly SJ, Hallstrom AP, Cappato R, Schron EB, Kuck K, Zipes D, Greene HL, Boczor S, Domanski M, Follmann D (2000): Meta-analysis of the implantable cardioverter defibrillator secondary prevention trials. AVID, CASH and CIDS studies. Antiarrhythmics vs implantable defibrillator study. Cardiac arrest study Hamburg. Canadian implantable defibrillator study. Eur Heart J $\underline{21}, 2071-2078$ 
Corrado D, Leoni L, Link MS, Della Bella P, Gaita F, Curnis A, Salerno JU, Igidbashian D, Raviele A, Disertori $\mathrm{M}$ et al. (2003): Implantable cardioverter-defibrillator therapy for prevention of sudden death in patients with arrhythmogenic right ventricular cardiomyopathy/dysplasia. Circulation $\underline{108}, 3084$ 3091

Corrado D, Calkins H, Link MS, Leoni L, Favale S, Bevilacqua M, Basso C, Ward D, Boriani G, Ricci R et al. (2010): Prophylactic implantable defibrillator in patients with arrhythmogenic right ventricular cardiomyopathy/dysplasia and no prior ventricular fibrillation or sustained ventricular tachycardia. Circulation 122, 1144-1152

Cowie MR, Marshall D, Drummond M, Ferko N, Maschio M, Ekman M, De Roy L, Heidbuchel H, Verboven Y, Braunschweig F et al. (2009): Lifetime cost-effectiveness of prophylactic implantation of a cardioverter defibrillator in patients with reduced left ventricular systolic function: results of markov modelling in a european population. Europace 11, 716-726

Dabiri Abkenari L, Theuns DA, Valk SDA, Belle Y, Groot NM, Haitsma D, Muskens-Heemskerk A, Szili-Torok T, Jordaens L (2011): Clinical experience with a novel subcutaneous implantable defibrillator system in a single center. Clin Res Cardiol $\underline{100}, 737-744$

Detsch D, Goerler H, Klein G, Haase H, Niehaus M (2006): Development of a rechargeable implantablecardioverter/defibrillator - first results of a long-term animal study. Heart Rhythm $\underline{3}, 41-42$

Ellinor PT, Guy ML, Ruskin JN, McGovern BA (2003): Variability in implantable cardioverter defibrillator pulse generator longevity between manufacturers. Pacing Clin Electrophysiol $\underline{26}, 71-75$

Ezekowitz JA, Armstrong PW, McAlister FA (2003): Implantable cardioverter defibrillators in primary and secondary prevention: a systematic review of randomized, controlled trials. Ann Intern Med $\underline{138}$, 445-452

Gould PA, Krahn AD (2006): Complications associated with implantable cardioverter-defibrillator replacement in response to device advisories. JAMA $\underline{295}, 1907-1911$

Guedon-Moreau L, Lacroix D, Sadoul N, Clementy J, Kouakam C, Hermida J, Aliot E, Boursier M, Bizeau O, Kacet S (2013): A randomized study of remote follow-up of implantable cardioverter defibrillators: safety and efficacy report of the ECOST trial. Eur Heart J $\underline{34}, 605-614$

Hauser RG (2005): The growing mismatch between patient longevity and the service life of implantable cardioverter-defibrillators. J Am Coll Cardiol 45, 2022-2025 
Hindricks G, Camm J, Merkely B, Raatikainen MJP, Arnar DO (2017): The EHRA white book 2017. the current status of electrophysiology in ESC member countries. Tenth edition.

https://www.escardio.org/static_file/Escardio/Subspecialty/EHRA/Publications/Documents/2017/ehrawhite-book-2017.pdf; abgerufen am 12.01.2020

Higgins SL, Yong P, Sheck D, McDaniel M, Bollinger F, Vadecha M, Desai S, Meyer DB (2000): Biventricular pacing diminishes the need for implantable cardioverter defibrillator therapy. J Am Coll Cardiol $\underline{36}, 824-827$

Hoffmann E, Steinbeck G (1998): Experience with pectoral versus abdominal implantation of a small defibrillator. a multicenter comparison in 778 patients. Eur Heart $\mathrm{J} \underline{19}, 1085-1098$

Horlbeck FW, Mellert F, Kreuz J, Nickenig G, Schwab JO (2012): Real-world data on the lifespan of implantable cardioverter-defibrillators depending on manufacturers and the amount of ventricular pacing. J Cardiovasc Electrophysiol 23, 1336-1342

Johansen JB, Jorgensen OD, Moller M, Arnsbo P, Mortensen PT, Nielsen JC (2011): Infection after pacemaker implantation: infection rates and risk factors associated with infection in a populationbased cohort study of 46299 consecutive patients. Eur Heart J $\underline{32}, 991-998$

Johansen JB, Nielsen JC, Sandgaard NCF (2016): Longevity of implantable cardioverterdefibrillators: still a long way to go. Europace $\underline{18}, 1285-1286$

Kaufman ES (2003): Saving lives in congenital long QT syndrome: who benefits from implantable cardioverter defibrillator therapy? J Cardiovasc Electrophysiol 14, 342-343

Knops P, Theuns DA, Res JC, Jordaens L (2009): Analysis of implantable defibrillator longevity under clinical circumstances: implications for device selection. Pacing Clin Electrophysiol. $\underline{32}, 1276-1285$

Krahn AD, Lee DS, Birnie D, Healey JS, Crystal E, Dorian P, Simpson CS, Khaykin Y, Cameron D, Janmohamed A et al. (2011): Predictors of short-term complications after implantable cardioverterdefibrillator replacement: results from the Ontario ICD database. Circ Arrhythm Electrophysiol $\underline{4}, 136-$ 142

Kuck K, Cappato R, Siebels J, Ruppel R (2000): Randomized comparison of antiarrhythmic drug therapy with implantable defibrillators in patients resuscitated from cardiac arrest: the cardiac arrest study Hamburg (CASH). Circulation 102, 748-754 
Landolina M, Curnis A, Morani G, Vado A, Ammendola E, D'onofrio A, Stabile G, Crosato M, Petracci $B$, Ceriotti $C$ et al. (2015): Longevity of implantable cardioverter-defibrillators for cardiac resynchronization therapy in current clinical practice: an analysis according to influencing factors, device generation, and manufacturer. Europace $\underline{17}, 1251-1258$

Lavergne T, Daubert JC, Chauvin M, Dolla E, Kacet S, Leenhardt A, Mabo P, Ritter P, Sadoul N, Saoudi $N$ et al. (1997): Preliminary clinical experience with the first dual chamber pacemaker defibrillator. Pacing Clin Electrophysiol $\underline{20}, 182-188$

Lee DS, Green LD, Liu PP, Dorian P, Newman DM, Grant F, Tu JV, Alter DA (2003): Effectiveness of implantable defibrillators for preventing arrhythmic events and death. J Am Coll Cardiol $\underline{41}, 1573-1582$

Littmann L, Monroe MH, Kerns W2, Svenson RH, Gallagher JJ (2003): Brugada syndrome and "brugada sign": clinical spectrum with a guide for the clinician. Am Heart J $\underline{145}, 768-778$

Manolis AS, Maounis T, Koulouris S, Vassilikos V (2017): "Real life" longevity of implantable cardioverter-defibrillator devices. Clin Cardiol $\underline{40}$, 759-764

Maron BJ, Shen W, Link MS, Epstein AE, Almquist AK, Daubert JP, Bardy GH, Favale S, Rea RF, Boriani $G$ et al. (2000): Efficacy of implantable cardioverter-defibrillators for the prevention of sudden death in patients with hypertrophic cardiomyopathy. N Engl J Med $\underline{342}, 365-373$

Mirowski M, Reid PR, Mower MM, Watkins L, Gott VL, Schauble JF, Langer A, Heilman MS, Kolenik SA, Fischell RE et al. (1980): Termination of malignant ventricular arrhythmias with an implanted automatic defibrillator in human beings. N Engl J Med $\underline{303}, 322-324$

Mond HG, Freitag G (2014): The cardiac implantable electronic device power source: evolution and revolution. Pacing Clin Electrophysiol $\underline{37}$, 1728-1745

Moss AJ, Hall WJ, Cannom DS, Daubert JP, Higgins SL, Klein H, Levine JH, Sanjeev S, Waldo AL, Wilber D (1996): Improved survival with an implanted defibrillator in patients with coronary disease at high risk for ventricular arrhythmia. N Engl J Med $\underline{335}, 1933-1940$

Moss AJ, Zareba W, Hall WJ, Klein H, Wilber DJ, Cannom DS, Daubert JP, Higgins SL, Brown MW, Andrews ML (2002): Prophylactic implantation of a defibrillator in patients with myocardial infarction and reduced ejection fraction. $\mathrm{N}$ Engl J Med $\underline{346}, 877-883$ 
Moss AJ, Hall WJ, Cannom DS, Klein H, Brown MW, Daubert JP, Estes NAM III, Foster E, Greenberg $\mathrm{H}$, Higgins SL et al. (2009): Cardiac-resynchronization therapy for the prevention of heart-failure events. N Engl J Med $\underline{361}, 1329-1338$

Moss AJ, Schuger C, Beck CA, Brown MW, Cannom DS, Daubert JP, Estes NAM III, Greenberg H, Hall WJ, Huang DT et al. (2012): Reduction in inappropriate therapy and mortality through ICD programming. N Engl J Med $\underline{367}, 2275-2283$

Neuzner J (2015): The mismatch between patient life expectancy and the service life of implantable devices in current cardioverter-defibrillator therapy: a call for larger device batteries. Clin Res Cardiol $\underline{104}, 456-460$

Ponikowski P, Voors AA, Anker SD, Bueno H, Cleland JG, Coats AJ, Falk V, González-Juanatey JR, Harjola VP, Jankowska EA et al. (2016): 2016 ESC guidelines for the diagnosis and treatment of acute and chronic heart failure: The task force for the diagnosis and treatment of acute and chronic heart failure of the european society of cardiology (ESC). Developed with the special contribution of the heart failure association (HFA) of the ESC. Eur Heart J $\underline{37}, 2129-2200$

Poole JE, Gleva MJ, Mela T, Chung MK, Uslan DZ, Borge R, Gottipaty V, Shinn T, Dan D, Feldman LA et al. (2010): Complication rates associated with pacemaker or implantable cardioverter-defibrillator generator replacements and upgrade procedures: results from the REPLACE registry. Circulation $\underline{122}$, 1553-1561

Priori SG, Borggrefe M, Camm AJ, Hauer RN, Klein H, Kuck KH, Schwartz PJ, Touboul P, Wellens HJ (1995): Role of the implantable defibrillator in patients with idiopathic ventricular fibrillation: data from the UCARE international registry. Pacing Clin Electrophysiol $\underline{18}, 799$

Priori SG, Maugeri FS, Matteo PS (1997): Survivors of out-of-hospital cardiac arrest with apparently normal heart. Need for definition and standardized clinical evaluation. Consensus statement of the joint steering committees of the unexplained cardiac arrest registry of Europe and of the idiopathic ventricular fibrillation registry of the United States. Circulation 95, 265-272

Priori SG, Aliot E, Blømstrom-Lundqvist C, Bossaert L, Breithardt G, Brugada P, Camm JA, Cappato R, Cobbe SM, Di MC (2001): Task force on sudden cardiac death of the european society of cardiology. Eur Heart J $\underline{22}, 1374-1450$

Priori SG, Napolitano C, Gasparini M, Pappone C, Della BP, Giordano U, Bloise R, Giustetto C, De Nardis R, Grillo M et al. (2002): Natural history of brugada syndrome: insights for risk stratification and management. Circulation $\underline{105}, 1342-1347$ 
Priori SG, Blomström-Lundqvist C, Mazzanti A, Blom N, Borggrefe M, Camm J, Elliott PM, Fitzsimons D, Hatala R, Hindricks G et al. (2015): 2015 ESC guidelines for the management of patients with ventricular arrhythmias and the prevention of sudden cardiac death: The task force for the management of patients with ventricular arrhythmias and the prevention of sudden cardiac death of the european society of cardiology (ESC). Endorsed by: association for european paediatric and congenital cardiology (AEPC). Eur Heart J $\underline{36}$, 2793-2867

Gandjour A, Holler A, Adarkwah CC (2011): Cost-effectiveness of implantable defibrillators after myocardial infarction based on 8-year follow-up data (MADIT II). Value Health $\underline{14}, 812-817$

Raatikainen MJP, Arnar DO, Merkely B, Nielsen JC, Hindricks G, Heidbuchel H, Camm J (2017): A decade of information on the use of cardiac implantable electronic devices and interventional electrophysiological procedures in the european society of cardiology countries: 2017 report from the european heart rhythm association. Europace $\underline{19}$, ii1-ii90

Ramachandra I (2010): Impact of ICD battery longevity on need for device replacements - insights from a veterans affairs database. Pacing Clin Electrophysiol $\underline{33}$, 314-319

Saksena S, Parsonnet V (1988): Implantation of a cardioverter/defibrillator without thoracotomy using a triple electrode system. JAMA $\underline{259}, 69-72$

Sarter BH, Callans DJ, Gottlieb GD, Schwartzman DS, Marchlinski FE (1998): Implantable defibrillator diagnostic storage capabilities: evolution, current status, and future utilization. Pacing Clin Electrophysiol 21, 1287-1298

Schaer BA, Koller MT, Sticherling C, Altmann D, Joerg L, Osswald S (2009): Longevity of implantable cardioverter-defibrillators, influencing factors, and comparison to industry-projected longevity. Heart Rhythm $\underline{6}, 1737-1743$

Shafat T, Baumfeld Y, Novack V, Konstantino Y, Amit G (2013): Significant differences in the expected versus observed longevity of implantable cardioverter defibrillators (ICDs). Clin Res Cardiol 102, 4349

Shannon KM (2002): Use of implantable cardioverter-defibrillators in pediatric patients. Curr Opin Cardiol $\underline{17}, 280-282$

Sheldon R, Connolly S, Krahn A, Roberts R, Gent M, Gardner M (2000): Identification of patients most likely to benefit from implantable cardioverter-defibrillator therapy: the canadian implantable defibrillator study. Circulation 101, 1660-1664 
Swygman C, Wang PJ, Link MS, Homoud MK, Estes NAM III (2002): Advances in implantable cardioverter defibrillators. Curr Opin Cardiol 17, 24-28

Tavernier R, Gevaert S, De Sutter J, De Clercq A, Rottiers H, Jordaens L, Fonteyne W (2001): Long term results of cardioverter-defibrillator implantation in patients with right ventricular dysplasia and malignant ventricular tachyarrhythmias. Heart Rhythm $\underline{85}, 53-56$

Thijssen J, Borleffs CJ, Van Rees JB, Man S, De Bie MK, Venlet J, Van der Velde ET, Van Erven L, Schalij MJ (2012): Implantable cardioverter-defibrillator longevity under clinical circumstances: an analysis according to device type, generation, and manufacturer. Heart Rhythm $\underline{9}, 513-519$

US Food and Drug Administration (1985): Cardiac Pacemakers, Inc.; Premarket approval of the automatic implantable cardioverter defibrillator (AICD) system. Fed Regist $\underline{50}, 47276$

Uslan DZ, Gleva MJ, Warren DK, Mela T, Chung MK, Gottipaty V, Borge R, Dan DA, Shinn T, Mitchell $\mathrm{K}$ et al. (2012): Cardiovascular implantable electronic device replacement infections and prevention: results from the REPLACE registry. Pacing Clin Electrophysiol $\underline{35}, 81-87$

Van Welsenes GH, Borleffs CJW, Van Rees JB, Atary JZ, Thijssen J, Van der Wall EE, Schalij MJ (2011): Improvements in 25 years of implantable cardioverter defibrillator therapy. Neth Heart J $\underline{19}$, 2430

Van Welsenes GH, Van Rees JB, Thijssen J, Trines SA, Van Erven L, Schalij MJ, Borleffs CJ (2011): Primary prevention implantable cardioverter defibrillator recipients: the need for defibrillator back-up after an event-free first battery service-life. J Cardiovasc Electrophysiol 22, $1346-1350$

Varma N, Love CJ, Schweikert R, Moll P, Michalski J, Epstein AE; TRUST Investigators (2018): Automatic remote monitoring utilizing daily transmissions: transmission reliability and implantable cardioverter defibrillator battery longevity in the TRUST trial. Europace $\underline{20}$, 622-628

Von Gunten S, Schaer BA, Yap S, Szili-Torok T, Kühne M, Sticherling C, Osswald S, Theuns DA (2016): Longevity of implantable cardioverter defibrillators: a comparison among manufacturers and over time. Europace 18, 710-717

Wever EF, Hauer RN, Van Capelle FL, Tijssen JG, Crijns HJ, Algra A, Wiesfeld AC, Bakker PF, De Robles Medina EO (1995): Randomized study of implantable defibrillator as first-choice therapy versus conventional strategy in postinfarct sudden death survivors. Circulation $\underline{91}, 2195-2203$ 
Wild DM, Fisher JD, Kim SG, Ferrick KJ, Gross JN, Palma EC (2004): Pacemakers and implantable cardioverter defibrillators: device longevity is more important than smaller size: the patient's viewpoint. Pacing Clin Electrophysiol 27, 1526-1529

Wilkoff BL, Cook, JR, Epstein AE, Greene HL, Hallstrom AP, Hsia H, Kutalek SP, Sharma A (2002): Dual-chamber pacing or ventricular backup pacing in patients with an implantable defibrillator: the dual chamber and VVI implantable defibrillator (DAVID) trial. JAMA $\underline{288}, 3115-3123$

Yap SC, Schaer BA, Bhagwandien RE, Kühne M, Dabiri Abkenari L, Osswald S, Szili-Torok T, Sticherling C, Theuns DA (2014): Evaluation of the need of elective implantable cardioverterdefibrillator generator replacement in primary prevention patients without prior appropriate ICD therapy. Heart $\underline{100}, 1188-1192$

Young JB, Abraham WT, Smith AL, Leon AR, Lieberman R, Wilkoff B, Canby RC, Schroeder JS, Liem LB, Hall S et al. (2003): Combined cardiac resynchronization and implantable cardioversion defibrillation in advanced chronic heart failure: the MIRACLE ICD Trial. JAMA $\underline{289}, 2685-2694$

Zanon F, Martignani C, Ammendola E, Menardi E, Narducci ML, De Filippo P, Santamaria M, Campana A, Stabile G, Potenza DR et al. (2016): Device longevity in a contemporary cohort of ICD/CRT-D patients undergoing device replacement. J Cardiovasc Electrophysiol 27, 840-845

Zareba W, Moss AJ, Daubert JP, Hall WJ, Robinson JL, Andrews M (2003): Implantable cardioverter defibrillator in high-risk long QT syndrome patients. J Cardiovasc Electrophysiol $\underline{14}$, 337-341

Zipes DP, Roberts D (1995): Results of the international study of the implantable pacemaker cardioverter-defibrillator. A comparison of epicardial and endocardial lead systems. The pacemakercardioverter-defibrillator investigators. Circulation $\underline{92}, 59-65$ 


\section{Danksagung}

An dieser Stelle möchte ich mich besonders bei Herrn Professor Dr. med. Markus Zabel für die Überlassung des Themas sowie die Betreuung der vorliegenden Arbeit bedanken. Außerdem danke ich Herrn PD Dr. med. Joachim Seegers für Anregungen und Korrekturen im Rahmen der Abfassung der Arbeit. Des Weiteren gilt mein Dank Herrn PD Dr. med. Marc Dorenkamp, insbesondere für die Betreuung und Hilfestellung bei der Datenerhebung in der Anfangsphase. Darüber hinaus danke ich Frau Sabine Busse für die Einführung in die praktische Arbeit der ICD-Ambulanz der Universitätsmedizin Göttingen. Nicht zuletzt gilt mein Dank dem Institut für Medizinische Statistik der Universitätsmedizin Göttingen für die Beratung bei der statistischen Analyse. 